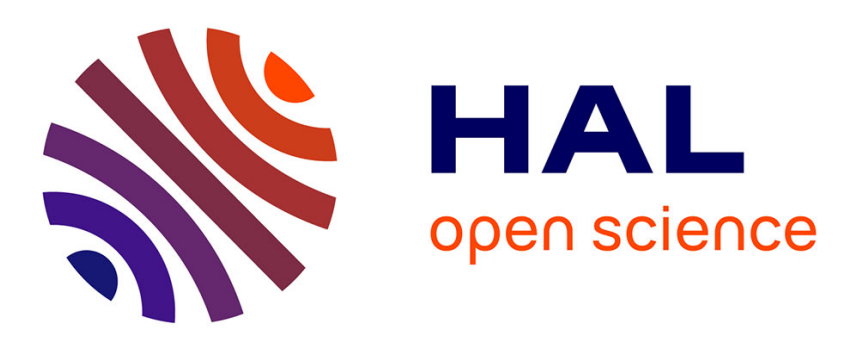

\title{
High frequency behavior of the Maxwell-Bloch model with relaxation: convergence to the Schrödinger-rate system
}

François Castella, Eric Dumas

\section{- To cite this version:}

François Castella, Eric Dumas. High frequency behavior of the Maxwell-Bloch model with relaxation: convergence to the Schrödinger-rate system. Journal of Hyperbolic Differential Equations, 2012, 9 (3), pp.355-401. 10.1142/S0219891612500129 . hal-00505587

\section{HAL Id: hal-00505587 \\ https://hal.science/hal-00505587}

Submitted on 24 Jul 2010

HAL is a multi-disciplinary open access archive for the deposit and dissemination of scientific research documents, whether they are published or not. The documents may come from teaching and research institutions in France or abroad, or from public or private research centers.
L'archive ouverte pluridisciplinaire HAL, est destinée au dépôt et à la diffusion de documents scientifiques de niveau recherche, publiés ou non, émanant des établissements d'enseignement et de recherche français ou étrangers, des laboratoires publics ou privés. 


\title{
High frequency behaviour of the Maxwell-Bloch model with relaxations: convergence to the Schrödinger-Boltzmann system
}

\author{
F. Castella ${ }^{(1)}$ and E. Dumas ${ }^{(2)}$ \\ (1) IRMAR, UMR 6625 (CNRS-UR1) \\ Université de Rennes 1 \\ Campus de Beaulieu, 35042 Rennes Cedex - France \\ email: francois.castella@univ-rennes1.fr \\ (2) Institut Fourier, UMR 5582 (CNRS-UJF) \\ 100 rue des Mathématiques \\ Domaine Universitaire \\ BP 74, 38402 Saint Martin d'Hères - France \\ email: edumas@ujf-grenoble.fr
}

\begin{abstract}
We study the Maxwell-Bloch model, which describes the propagation of a laser through a material and the associated interaction between laser and matter (polarization of the atoms through light propagation, photon emission and absorption, etc.). The laser field is described through Maxwell's equations, a classical equation, while matter is represented at a quantum level and satisfies a quantum Liouville equation known as the Bloch model. Coupling between laser and matter is described through a quadratic source term in both equations. The model also takes into account partial relaxation effects, namely the trend of matter to return to its natural thermodynamic equilibrium. The whole system involves $6+N(N+1) / 2$ unknowns, the six-dimensional electromagnetic field plus the $N(N+1) / 2$ unknowns describing the state of matter, where $N$ is the number of atomic energy levels of the considered material.

We consider at once a high-frequency and weak coupling situation, in the general case of anisotropic electromagnetic fields that are subject to diffraction. Degenerate energy levels are allowed. The whole system is stiff and involves strong nonlinearities.

We show the convergence to a nonstiff, nonlinear, coupled Schrödinger-Boltzmann model, involving $3+N$ unknowns. The electromagnetic field is eventually described through its envelope, one unknown vector in $\mathbb{C}^{3}$. It satisfies a Schrödinger equation that takes into account propagation and diffraction of light inside the material. Matter on the other hand is described through a $N$-dimensional vector describing the occupation numbers of each atomic level. It satisfies a Boltzmann equation that describes the jumps of the electrons between the various atomic energy levels, as induced by the interaction with light. The rate of exchange between the atomic levels is proportional to the intensity of the laser field. The whole system is the physically natural nonlinear model.

In order to provide an important and explicit example, we completely analyze the specific (two dimensional) Transverse Magnetic case, for which formulae turn out to be simpler.

Technically speaking, our analysis does not enter the usual mathematical framework of geometric optics: it is more singular, and requires an ad hoc Ansatz.
\end{abstract}




\section{Contents}

1 Introduction $\quad 2$

2 Presentation of the results 1

2.1 The model . . . . . . . . . . . . . . . . . . . . .

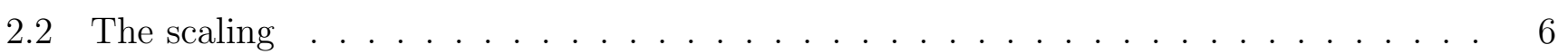

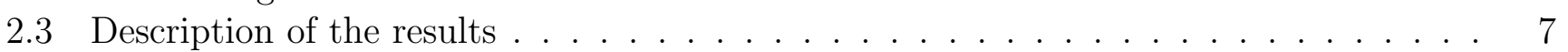

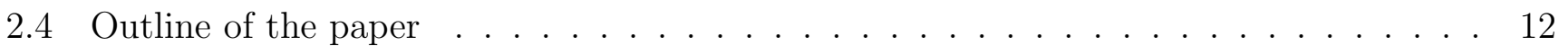

3 Formulating the Ansatz $\quad 13$

4 Formal expansions and approximate solution 15

4.1 Rapid modes and algebraic projectors . . . . . . . . . . . . . . . 17

4.2 Profile equations, fast scale analysis . . . . . . . . . . . . . . . . . . . . . 19

4.2 .1 The residual $r^{-2} \ldots \ldots \ldots \ldots \ldots \ldots \ldots$

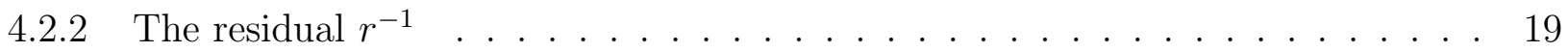

4.2 .3 The residual $r^{0} \ldots \ldots \ldots \ldots \ldots \ldots \ldots$

4.3 Profile equations, intermediate scale analysis . . . . . . . . . . . . . 21

4.3.1 Analysis of fields and populations: average operators . . . . . . . . . . . 22

4.3.2 Analysis of coherences: exponential growth . . . . . . . . . . . . . 25

4.4 Solving the profile equations . . . . . . . . . . . . . . . . . 26

4.4.1 Computing the dominant profile $\mathbf{U}^{0} \ldots \ldots \ldots \ldots \ldots$

4.4.2 Computing the correctors $\mathbf{U}^{1}$ and $\mathbf{U}^{2} \ldots \ldots \ldots \ldots \ldots$

5 Convergence $\quad 31$

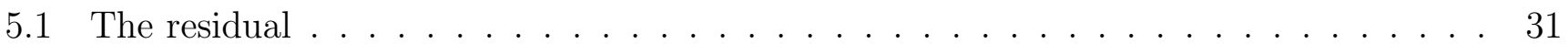

5.2 Stability . . . . . . . . . . . . . . . . . . . 32

6 The Transverse Magnetic case 35

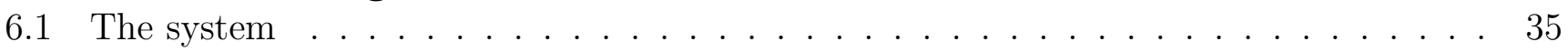

6.2 The Ansatz . . . . . . . . . . . . . . . . . . . . . 36

6.3 WKB expansions . . . . . . . . . . . . . . . . . . . . 36

6.4 Conclusion in the TM case . . . . . . . . . . . . . . . . . . 38

Support by the program "SYDYQ: SYstèmes DYnamiques Quantiques" from the Université Joseph Fourier (Grenoble) is acknowledged.

\section{Introduction}

Maxwell-Bloch systems are of common use in Laser Physics (see the textbooks [8], [10], [16], [34], [36], 37]). They modelize the evolution of an electromagnetic field, treated classically, and coupled with an ensemble of identical atoms, which in turn are described by a quantum density matrix. This model is relevant when atoms are far from the ionization energy (to possess discrete energy levels), while they have sufficiently low density and the laser field is strong enough (which allows to describe the field classically while matter is described in a quantum way - see [16]). 
In the Maxwell-Bloch model, the electromagnetic field satisfies Maxwell's equations, whose unknowns are the electric and magnetic fields $E \in \mathbb{R}^{3}$ and $B \in \mathbb{R}^{3}$, where $E=E(t, x, y, z)$ and $B=B(t, x, y, z)$ and $t \in \mathbb{R}$ is time while $(x, y, z) \in \mathbb{R}^{3}$ are the space coordinates. Matter is described through a Bloch equation, whose unknown is the density matrix $\rho=\rho(t, x, y, z)$, a quantum variable which describes the atomic state at $(t, x, y, z)$. We consider that the atoms only visit the $N$ lowest energy levels. The latter are the $N$ first eigenstates of the free material system, in the absence of fields. In this basis, the density matrix $\rho=\rho(t, x, y, z)$ is an $N \times N$ matrix, for each value of $(t, x, y, z)$. The diagonal entries $\rho(t, x, y)(n, n)$ (called populations) give the proportion of matter that lies in the $n$-th energy level $(n=1, \ldots, N)$, while the off-diagonal entries $\rho(t, x, y, z)(n, p)$ with $n \neq p$, (called coherences) give the correlation between levels $n$ and $p$. The complete Maxwell-Bloch system takes into account the coupling between the laser field and the atoms via terms that are quadratic, proportional to $\rho \times E$, and which describe polarization of matter due to laser propagation.

We study the high frequency and weak coupling behaviour of the Maxwell-Bloch system, a situation in which the typical frequencies of the field and of the atoms' oscillations are large and possibly resonate, while the strength of laser-matter coupling is small.

For large frequencies, the electromagnetic field is expected to be asymptotically solution to a nonlinear Schrödinger equation. This is the paraxial approximation. We refer to [18 and 26], as well as [30] on these matters, when the sole laser field propagates (no coupling with matter). When matter is actually coupled to the field, we refer to [9]. Here a high frequency Maxwell-Bloch system is studied both physically and mathematically, for atoms that only possess 3 non-degenerate energy levels. The analysis leads to a Schrödinger-Bloch approximation of the original system, in a spirit similar to the present paper.

The weak coupling behaviour of matter is a bit more delicate to handle: to have a clean limit, one needs to take thermodynamic fluctuations into account. For this reason we introduce, in a standard fashion, phenomenological relaxation operators in the original Bloch system. These impose a rapid decay of coherences, as well as a quick return to equilibrium of populations. We refer to [3] for mathematical properties of the relaxation operators that are natural in this context. Due to the relaxation effects, it is expected that Bloch's equation is asymptotic to a Boltzmann equation, sometimes called "Einstein's rate equation" ( $c f$. [33], [4]). It describes how the atoms jump between the various energy levels under the action of the external field. When the driving high frequency field is given (and the Bloch model is thus linear), we refer to the papers [6] and [7], which study the actual convergence of Bloch's equation to a Boltzmann model in the weak coupling regime. In that case, a formula is found for the transition rates involved in the limiting Einstein rate equation, which coincides with the one formally obtained in the Physics literature. Note however that the question studied in [6] and [7] is a linear problem, and proofs strongly use ODE averaging techniques as well as the positivity of relaxation operators (features that the present text does not share). We also mention [5], where similar asymptotics are treated both for quantum and classical models. We stress finally that many other works deal with the rigorous derivation of Boltzmann like equations from (usually linear) models describing the interaction of waves/particles with external media. A non-convergence result is given in [14] and [15]. Convergence in the case of an electron in a periodic box is studied in [11], [12], [13], while the case of an electron in a random medium is addressed in [22], 29], [38], [39, 40] - see also [35] for a semi-classical approach. In a nonlinear context, a partial result is obtained in [2].

The above formal discussion suggests, in the present case, that the high-frequency Maxwell system goes to a Schrödinger model for the envelope of the field, while the weakly coupled Bloch system supposedly goes to a Boltzmann equation describing the jumps of electrons between the atomic levels. 
This is the program we rigorously develop in the present paper. We fully prove convergence of the coupled Maxwell-Bloch system to a coupled Schrödinger-Boltzmann model. We also prove that the rate of exchange between the energy levels is proportional to the laser's intensity. In doing so we recover the physically relevant model. Our approach mainly uses three-scales geometric optics, yet in a more singular context where the partial relaxation effects impose a specific treatment of coherences.

\section{Presentation of the results}

\section{$2.1 \quad$ The model}

The Maxwell-Bloch system, whose unknowns are the electric field $E=E(t, x, y, z) \in \mathbb{R}^{3}$, the magnetic field $B=B(t, x, y, z) \in \mathbb{R}^{3}$, together with the density matrix $\rho=\rho(t, x, y, z) \in \mathbb{C}^{N \times N}$ (the space of $N \times N$ complex matrices), reads

$$
\begin{aligned}
& \partial_{t} B+\operatorname{curl} E=0, \\
& \partial_{t} E-\operatorname{curl} B=-\partial_{t} P, \quad \text { with } P=\operatorname{Tr}(\Gamma \rho), \\
& \partial_{t} \rho=-i[\Omega-E \cdot \Gamma, \rho]+Q(\rho), \\
& \text { where } Q(\rho)=W \sharp \rho_{\mathrm{d}}-\gamma \rho_{\text {od }} .
\end{aligned}
$$

In these equations, curl is the usual curl operator on vector fields in $\mathbb{R}^{3}, \Gamma$ as well as $\Omega$ are given matrices in $\mathbb{C}^{N \times N}, \gamma>0$ is a given positive constant, and whenever $A$ and $B$ are matrices in $\mathbb{C}^{N \times N}$, the trace $\operatorname{Tr}(A)$ denotes the usual trace of $A$ while the bracket $[A, B]$ denotes the usual commutator between matrices

$$
[A, B]=A B-B A .
$$

The term $Q(\rho)=Q(\rho)(t, x, y, z)$ is the so-called relaxation matrix, an $N \times N$ matrix for each value of $(t, x, y, z)$. Its definition involves $W \in \mathbb{C}^{N \times N}$, a given matrix with nonnegative entries $W(n, m) \geq 0$, while $\rho_{\mathrm{d}}$ and $\rho_{\text {od }}$ denote the diagonal respectively off-diagonal parts of the density matrix $\rho$ (they correspond respectively to the populations and the coherences). They are $N \times N$ matrices defined, for each value of $(t, x, y, z)$, by their entries

$$
\begin{aligned}
& \rho_{\mathrm{d}}(t, x, y, z)(n, p)=\rho(t, x, y, z)(n, p) \mathbf{1}[n=p], \\
& \rho_{\mathrm{od}}(t, x, y, z)(n, p)=\rho(t, x, y, z)(n, p) \mathbf{1}[n \neq p] .
\end{aligned}
$$

Equation (4) also uses the following notation, valid thoughout the present text : given any matrix $A$ with nonnegative entries we set

$$
\left\{\begin{array}{l}
A \sharp \rho_{\mathrm{d}}(n, n)=\sum_{k=1}^{N}\left[A(k, n) \rho_{\mathrm{d}}(k, k)-A(n, k) \rho_{\mathrm{d}}(n, n)\right], \\
A \sharp \rho_{\mathrm{d}}(n, p)=0 \quad \text { when } n \neq p .
\end{array}\right.
$$

The meaning of operator $Q(\rho)$ in (3) is the following. The term $-\gamma \rho_{\text {od }}$ induces exponential relaxation to zero for the coherences, while the term $W \sharp \rho_{\mathrm{d}}$ acts on the populations only, and induces exponential relaxation of the populations towards some thermodynamical equilibrium that depends on the values of the $W(n, p)$ 's. As in conventional kinetic theory of gases, relation (5) asserts that along time evolution, atoms may leave with probability $W(k, n)$ the $k$ th eigenstate to populate the $n$th eigenstate 
(this is the so-called gain term $\sum_{k=1}^{N} W(k, n) \rho_{\mathrm{d}}(k, k)$ ), while some atoms may conversely leave with probability $W(n, k)$ the $n$th state to populate some other $k$ th state (this is the so-called loss term $\left.-\sum_{k=1}^{N} W(n, k) \rho_{\mathrm{d}}(n, n)\right)$.

Theoretically, the Maxwell-Bloch system needs to be supplemented with the Ampère and Faraday laws,

$$
\operatorname{div} B=0, \quad \operatorname{div}(E+P)=0 .
$$

These constraints (6) are anyhow transported as soon as they are satisfied by the initial data, hence we shall skip them in the sequel.

Note that the above equations are readily given in the convenient dimensionless form that suits our purpose. The precise scaling under study is discussed later.

We now comment on these equations, and on all involved quantities.

The density matrix $\rho(t, x, y, z)$ is Hermitian and positive. It describes the state of matter at $(t, x, y, z)$.

The constant matrix $\Omega$ is the free Hamiltonian of the material system, written in the natural eigenbasis. It is a fixed physical constant associated with the considered atomic species. It reads

$$
\Omega=\operatorname{diag}(\omega(1), \ldots, \omega(N))
$$

where $0<\omega(1) \leq \cdots \leq \omega(N)$ are the atomic energies. For later convenience, we readily introduce the differences between energy levels, as

$$
\omega(n, k)=\omega(n)-\omega(k) .
$$

The constant matrix $\Gamma$ is called the dipolar operator. It is a hermitian matrix. It has the value $N_{\text {atomic }} \times \gamma_{\text {atomic }}$, where $N_{\text {atomic }}$ is the number of atoms per unit volume, while $\gamma_{\text {atomic }}$ is a fixed physical constant (a matrix) associated with the considered atomic species.

The entries of so-called dipolar momentum $E \cdot \Gamma$ are defined for any $m, n=1, \ldots, N$, as $(E$. $\Gamma)(t, x, y, z)(m, n)=E(t, x, y, z) \cdot \Gamma(m, n)$, where $a \cdot b$ denotes the componentwise product of two vectors in $\mathbb{C}^{3}$, namely

$$
(E \cdot \Gamma)(t, x, y, z)(m, n)=E_{x}(t, x, y, z) \Gamma(m, n)_{x}+E_{y}(t, x, y, z) \Gamma(m, n)_{y}+E_{z}(t, x, y, z) \Gamma(m, n)_{z},
$$

and the subscripts $x$ (resp. $y$, resp. $z$ ), denote the $x$ (resp. $y$, resp. $z$ ) components of the relevant vectors (note the absence of complex conjugation).

The non-negative transition coefficients $W(n, k) \geq 0$ are known as the Pauli coefficients, see [10]. They satisfy a micro-reversibility relation at temperature $T$, i.e. $W(n, k)=W(k, n) \exp (\omega(k, n) / T)$. In that perspective the relaxation operator $Q(\rho)$ translates the fact that the atoms tend to relax towards the thermodynamical equilibrium given by $\rho_{\text {od }} \equiv 0$ and $\rho_{\mathrm{d}}(n, n) \equiv \exp (-\omega(n) / T)$. Both the off-diagonal relaxation term $\gamma$ and the Pauli coefficients $W(n, k)$ are physical data. Contrary to $\Gamma$ or $\Omega$, their relation with given physical constants attached with the specific atomic species at hand is unclear.

The Bloch equation (3) relies on the so-called dipolar approximation : the coupling between light and matter is taken into account through the simplest $[E \cdot \Gamma, \rho]$ term, which is quadratic (proportional to $\rho \times E$ ) and local (it only depends on the value of $E$ and $\rho$ and the same point $(t, x, y, z)$ ). This approximation implicitely assumes that the wavelength of the field is larger than the typical spatial extension of the atom, hence can be taken constant over the whole domain occupied by each given atom. 


\subsection{The scaling}

Let us introduce the physical scales in the model, which transform all constants $(\Gamma, \Omega, \gamma, W)$ and unknowns $(E, B, \rho)$ into quantities of order one in the regime we wish to study.

Firstly, concerning the time variations, we study a high frequency regime. Calling $1 / \varepsilon$ the dimensionless parameter measuring typical values of the frequencies, i.e. the ratio between the time scale of observations and the time scale of the variations of $E, B$ and $\rho$, all time derivatives $\partial_{t}$ then become $\varepsilon \partial_{t}$ in the scaled version of (1)-(4).

Secondly, we want to study a situation where constructive interference occurs between the time oscillations of the electromagnetic field and the ones of the atom (giving rise to atomic absorption and emission of photons). For that reason, the time variations of $E, B$, and $\rho$ should all take place at similar frequencies. For that reason $\Omega$ is naturally a quantity of order one in the scaled model.

Thirdly, the typical strength of the coupling between light and matter is entirely determined by the physical constant $\Gamma$. Since weak coupling is realized when the polarization operator has an effect of the order one on the chosen time scale, it turns out that we need to prescribe $\Gamma=\mathcal{O}(\sqrt{\varepsilon})$. Mathematically, this means we shall replace $\Gamma$ by $\sqrt{\varepsilon} \Gamma$ in the scaled model. Indeed, the electric dipole momentum then becomes $|E \cdot(\sqrt{\varepsilon} \Gamma)|^{2}=\mathcal{O}(\varepsilon)$ which, integrated over macroscopic time scales $\mathcal{O}(1 / \varepsilon)$, results in an energy contribution of order one as desired. Physically, since $\Gamma=N_{\text {atomic }} \times \gamma_{\text {atomic }}$ where $N_{\text {atomic }}$ is the number of atoms per unit volume and $\gamma_{\text {atomic }}$ is the polarizability of one atom, this means that the atomic density is here tuned to be of order $\mathcal{O}(\sqrt{\varepsilon})$, so as to observe order one effect of coupling over this scale.

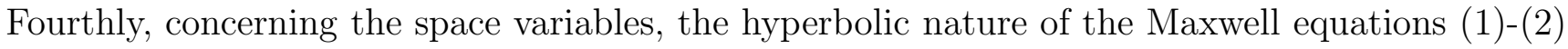
suggests to rescale space so that $\partial_{x}, \partial_{y}, \partial_{z}$ become $\varepsilon \partial_{x}, \varepsilon \partial_{y}$, and $\varepsilon \partial_{z}$, respectively, due to the scaling $\partial_{t} \mapsto \varepsilon \partial_{t}$ and to finite propagation speed: the laser visits space scales $\mathcal{O}(1 / \varepsilon)$ over time scales $\mathcal{O}(1 / \varepsilon)$. This simple scaling would in fact be easily described along lines similar to the present analysis. We wish to investigate physically richer situations where diffraction occurs. To this end, as in [9], [19] and [20], we impose anisotropy and introduce a third scale: on the one hand, we choose one direction of propagation, say the $x$ direction, meaning that at the microscopic scale, the fields vary with $x$ but not with $(y, z)$; on the other hand, we restrict our attention to fields that slowly vary over the scale $1 / \varepsilon$ in the $x$ direction (or, in other words, that have spatial extension $1 / \varepsilon$ in $x$ ), while they slowly vary over the scale $1 / \sqrt{\varepsilon}$ in the $(y, z)$ direction (or, in other words, that have spatial extension $1 / \sqrt{\varepsilon}$ in $(y, z))$. The typical shape of the laser beam is thus that of a "light cigar", as in [17]. In macroscopic scales, this provides fields that strongly oscillate at frequency $1 / \varepsilon$ in $x$ (and only in this direction), while they have support of size 1 in $x$, and support of size $\sqrt{\varepsilon}$ in $(y, z)$. All this imposes to rescale $\partial_{x}$ as $\varepsilon \partial_{x}$, and $\left(\partial_{y}, \partial_{z}\right)$ as $\left(\sqrt{\varepsilon} \partial_{y}, \sqrt{\varepsilon} \partial_{z}\right)$ in the above equations. We stress that this is a definite choice of shape of the kind of laser beams we wish to study. It is also a choice of polarization: these beams are shot in the $x$ direction only.

Fifthly, consider relaxations $Q(\rho)$. In order for the diagonal relaxation $W \sharp \rho_{\mathrm{d}}$ to have an $\mathcal{O}(1)$ effect at times $t$ of order $\mathcal{O}(1)$, we take the coefficients in $W$ of size $\varepsilon$, and write $\varepsilon W(n, k)$ instead of $W(n, k)$. The off-diagonal relaxation $-\gamma \rho_{\text {od }}$ on the other hand is supposed to have a much shorter time scale, which we choose to be of order $\mathcal{O}(\varepsilon)$. This corresponds to an off-diagonal relaxation $-\gamma \rho_{\text {od }}$ that remains unscaled. In fact, there is no theoretical description of the relaxation time as a function of $\varepsilon$. In [6] and [7], the off-diagonal relaxation is scaled as $-\gamma \varepsilon^{\mu} \rho_{\text {od }}$ with an extra free parameter $\mu$ that is constrained to satisfy $0 \leq \mu<1$ (to have a clean limit, off-diagonal relaxation should be strong enough with respect to the chosen time scale). For technical reasons, in these papers, $\mu$ is actually restricted to $0 \leq \mu<1 / 4$. In the present paper, the formal analysis could be performed for $0 \leq \mu<1$, yet our main stability result (Theorem 5.2) requires the stronger constraint 
$\mu=0$.

After rescaling all variables and physical constants accordingly, the system (1)-(团) becomes

$$
\left\{\begin{array}{l}
\partial_{t} B^{\varepsilon}+\operatorname{curl}_{\varepsilon} E^{\varepsilon}=0, \\
\partial_{t} E^{\varepsilon}-\operatorname{curl}_{\varepsilon} B^{\varepsilon}=\frac{i}{\sqrt{\varepsilon}} \operatorname{Tr}\left(\Gamma \Omega_{\gamma} \mathbf{C}^{\varepsilon}\right)-i \operatorname{Tr}\left(\Gamma\left[E^{\varepsilon} \cdot \Gamma, \mathbf{C}^{\varepsilon}+\mathbf{N}^{\varepsilon}\right]\right)-\sqrt{\varepsilon} \operatorname{Tr}\left(\Gamma W \sharp \mathbf{N}^{\varepsilon}\right), \\
\partial_{t} \mathbf{C}^{\varepsilon}=-\frac{i}{\varepsilon} \Omega_{\gamma} \mathbf{C}^{\varepsilon}+\frac{i}{\sqrt{\varepsilon}}\left[E^{\varepsilon} \cdot \Gamma, \mathbf{C}^{\varepsilon}+\mathbf{N}^{\varepsilon}\right]_{\mathrm{od}}, \\
\partial_{t} \mathbf{N}^{\varepsilon}=\frac{i}{\sqrt{\varepsilon}}\left[E^{\varepsilon} \cdot \Gamma, \mathbf{C}^{\varepsilon}\right]_{\mathrm{d}}+W \sharp \mathbf{N}^{\varepsilon} .
\end{array}\right.
$$

Here and in the sequel, we adopt for convenience the notation $\mathbf{C}^{\varepsilon}$ and $\mathbf{N}^{\varepsilon}$ for coherences $\rho_{\text {od }}$ and populations $\rho_{\mathrm{d}}$, respectively. Besides, throughout the sequel, subscripts "d" and "od" shall always refer to diagonal and off-diagonal parts of the considered $N \times N$ matrices. Lastly, we also denote by $\operatorname{curl}_{\varepsilon}$ the curl operator associated with our scaling, i.e.

$$
\operatorname{curl}_{\varepsilon} E=\left(\frac{1}{\sqrt{\varepsilon}} \partial_{y} E_{z}-\frac{1}{\sqrt{\varepsilon}} \partial_{z} E_{y}, \frac{1}{\sqrt{\varepsilon}} \partial_{z} E_{x}-\partial_{x} E_{z}, \partial_{x} E_{y}-\frac{1}{\sqrt{\varepsilon}} \partial_{y} E_{x}\right),
$$

and $\Omega_{\gamma}$ is a shorthand notation for $\operatorname{ad}_{\Omega}-i \gamma$,

$$
\Omega_{\gamma} \mathbf{C}=[\Omega, \mathbf{C}]-i \gamma \mathbf{C}, \quad \text { i.e. }\left(\Omega_{\gamma} \mathbf{C}\right)(n, p)=\omega(n, p) \mathbf{C}(n, p)-i \gamma \mathbf{C}(n, p) .
$$

\subsection{Description of the results}

Main result: profiles, separation of scales, and obtention of an approximate solution. Maxwell-Bloch's system (9) is a nonlinear hyperbolic symmetric system, singular in $\varepsilon$, that we may write symbolically

$$
L\left(\partial_{t}, \partial_{x}, \frac{1}{\sqrt{\varepsilon}} \partial_{y}, \frac{1}{\sqrt{\varepsilon}} \partial_{z}\right) \mathbf{U}^{\varepsilon}=F^{\varepsilon}\left(\mathbf{U}^{\varepsilon}\right) .
$$

Its unknown is

$$
\mathbf{U}^{\varepsilon}=\left(\mathbf{u}^{\varepsilon}, \mathbf{C}^{\varepsilon}, \mathbf{N}^{\varepsilon}\right)
$$

where $\mathbf{u}^{\varepsilon}$ stands for the sole electromagnetic field

$$
\mathbf{u}^{\varepsilon}=\left(B^{\varepsilon}, E^{\varepsilon}\right)
$$

We are interested in solving a Cauchy problem associated with (9) for initial data that are smooth, but high frequency, of order $\mathcal{O}(1 / \varepsilon)$. The typical difficulty is to ensure existence of the whole family $\left(\mathbf{U}^{\varepsilon}\right)_{\left.\varepsilon \in] 0, \varepsilon_{0}\right]}$ on some time interval $\left[0, t_{\star}\right]$, independent of $\varepsilon$.

This enters the framework of 3-scales diffractive optics (see [18], [30], and the surveys [27], [21]). To this end, our analysis uses a WKB analysis, based on profiles that we expand in successive powers of $\varepsilon$. An important original point yet is, we consider amplitudes that are even higher (namely $\mathcal{O}(1)$ ) than the ones allowed by transparency properties (namely $\mathcal{O}(\varepsilon)-$ see [28]). We show these larger amplitudes are eventually compensated by the partial relaxation: remember the sole equation on the coherences $\mathbf{C}^{\varepsilon}$ carries the relaxation term $-\gamma \mathbf{C}^{\varepsilon} / \varepsilon$ in (9). We refer to remark 3.2 below on this important point. 
We start with a given a wavevector

$$
k=\left(k^{1}, \ldots, k^{d}\right) \in \mathbb{R}^{d},
$$

and initial data of the form

$$
\mathbf{U}_{\text {ini }}^{\varepsilon}(x, y, z)=\underline{\mathbf{U}^{0}}\left(x, y, z, \frac{k x}{\varepsilon}\right)+\delta^{\varepsilon}\left(x, y, z, \frac{k x}{\varepsilon}\right) .
$$

The profiles $\underline{\mathbf{U}^{0}}\left(x, y, z, \theta_{0}\right), \delta^{\varepsilon}\left(x, y, z, \theta_{0}\right) \in \mathcal{C}^{\infty}\left(\mathbb{R} \times \mathbb{T}^{d}\right)$ that are periodic in $\theta_{0} \in \mathbb{R}^{d}$ and we assume that

$$
\delta^{\varepsilon} \underset{\varepsilon \rightarrow 0}{\longrightarrow} 0
$$

in every Sobolev space $H^{s}$. We also assume that $\underline{\mathbf{U}^{0}}$ is submitted to polarization conditions, namely that the electromagnetic field $\mathbf{u}^{0}$ has the usual directional constraints, see (35), and that some preferred components $\underline{\mathbf{C}^{0}}(m, n)$ vanish, see (36).

We build for all $\varepsilon>0$ an approximate solution $\mathbf{U}_{\text {app }}^{\varepsilon}$ to (9), defined on some time interval $\left[0, t_{\star}\right]$, and with initial values $\underline{\mathbf{U}^{0}}(x, y, z, k x / \varepsilon)$. Our construction uses a leading profile $\mathbf{U}^{0}$ and two correctors $\mathbf{U}^{1}, \mathbf{U}^{2}$, and $\mathbf{U}_{\text {app }}^{\varepsilon}$ is of the form

$$
\mathbf{U}_{\mathrm{app}}^{\varepsilon}(t, x, y, z)=\left.\sum_{j=0}^{2} \sqrt{\varepsilon}^{j} \mathbf{U}^{j}\left(t, x, y, z, T, \sigma, \theta_{0}, \theta_{1}\right)\right|_{T=t / \sqrt{\varepsilon}, \sigma=\gamma t / \varepsilon,\left(\theta_{0}, \theta_{1}\right)=(k x / \varepsilon,-k t / \varepsilon)} .
$$

Correctors $\mathbf{U}^{1}, \mathbf{U}^{2}$, are introduced so as to ensure consistancy of this Ansatz, namely

$$
L\left(\partial_{t}, \partial_{x}, \frac{1}{\sqrt{\varepsilon}} \partial_{y}, \frac{1}{\sqrt{\varepsilon}} \partial_{z}\right) \mathbf{U}_{\mathrm{app}}^{\varepsilon}-F^{\varepsilon}\left(\mathbf{U}_{\mathrm{app}}^{\varepsilon}\right)=\mathcal{O}(\sqrt{\varepsilon}) .
$$

One main point in our analysis is the separation of scales, and the crucial introduction of the fast variables $T, \sigma$ and $\theta_{1}$ in (13). Explanations on this point are postponed to the next paragraph.

Once the approximate solution is constructed, we prove Theorem 5.2. It asserts that, for $\varepsilon_{0}>0$ small enough, there is a unique solution $\mathbf{U}^{\varepsilon}$ to system (9) with initial value $\mathbf{U}_{\text {ini }}^{\varepsilon}$, which is well approximated by $\mathbf{U}_{\text {app }}^{\varepsilon}$, namely

$$
\forall \mu \in \mathbb{N}^{3}, \quad\left\|\partial_{x, y, z}^{\mu}\left(\mathbf{U}^{\varepsilon}-\mathbf{U}_{\text {app }}^{\varepsilon}\right)\right\|_{L^{\infty}\left(\left[0, t_{\star}\right] \times \mathbb{R}^{3}\right) \underset{\varepsilon \rightarrow 0}{\longrightarrow} 0 .} 0 .
$$

On top of that, we are able to completely describe the dynamics of the dominant term $\mathbf{U}^{0}$. It provides an $o(1)$ approximation of the original dynamics of $\mathbf{U}^{\varepsilon}$. The function $\mathbf{U}^{0}$ satisfies a coupled, nonlinear, Schrödinger-Bloch system, which we describe later.

This result is achieved via a singular system method (see [25]), where the unknown is a profile (thus with non-singular initial data). The difficulty comes from the "supersingular" nature of the system, and the fact that relaxations are only partial ones (acting on a part of the dependent variables only). This problem is overcome thanks to the structure of the approximate solution.

Describing the asymptotic dynamics (1) - oscillations and initial layer. In the chosen Ansatz (13), we assume that each profile $\mathbf{U}^{j}$ may be decomposed into modes $\mathbf{U}_{\alpha}^{j, \kappa}$, as

$$
\mathbf{U}^{j}(t, x, y, z, T, \sigma, \theta)=\sum_{\alpha \in \mathbb{Z}^{2 d}} \sum_{\kappa \in \mathbb{N}} \mathbf{U}_{\alpha}^{j, \kappa} \exp (i \alpha \cdot \theta) \exp (-\kappa \sigma)
$$


This defines the quantities $\mathbf{U}_{\alpha}^{j, \kappa}=\mathbf{U}_{\alpha}^{j, \kappa}(t, x, y, z, T)$, for which $\alpha \in \mathbb{Z}^{2 d}$ is seen as a Fourier mode, while $\kappa \in \mathbb{N}$ is seen as an exponentially decaying mode. We introduce in passing and for later convenience the notation

$$
\mathbf{U}_{\alpha}^{j}=\sum_{\kappa \in \mathbb{N}} \mathbf{U}_{\alpha}^{j, \kappa} \exp (-\kappa \sigma)
$$

a function of $(t, x, y, z, T, \sigma)$, as well as

$$
\mathbf{U}^{j, \kappa}=\sum_{\alpha \in \mathbb{Z}^{2 d}} \mathbf{U}_{\alpha}^{j, \kappa} \exp (i \alpha \cdot \theta)
$$

a function of $(t, x, y, z, T, \theta)$. For $\kappa>0$, the decomposition (16) encodes an exponential mode in the $\sigma=\gamma t / \varepsilon$ variable, representative of the initial layer induced by off-diagonal relaxations, while Fourier modes $\alpha=\left(\alpha_{0}, \alpha_{1}\right)$ reflect oscillations in the variable $\left(\theta_{0}, \theta_{1}\right)=(k x / \varepsilon,-k t / \varepsilon)$.

This choice of Ansatz is motivated by the following. The oscillations in $k x / \varepsilon$ are anyhow present in the initial data, and the hyperbolic feature of the equations ensures they are propagated into oscillations in the variable $(k x / \varepsilon,-k t / \varepsilon)$. Nonlinear interaction of waves next makes sure that all harmonics are created along time evolution in (9). To be more precise, we show that all oscillations that are characteristic for the Maxwell-Bloch system are propagated. They are given, for fields and populations, by the characteristic set

$$
\mathcal{C}:=\mathcal{C}_{+} \cup \mathcal{C}_{-} \cup \mathcal{C}_{0}
$$

where we define

$$
\mathcal{C}_{ \pm}=\left\{\alpha=\left(\alpha_{0}, \alpha_{1}\right) \in \mathbb{Z}^{2 d} \backslash\{0\} \mid \alpha_{1}= \pm \alpha_{0}\right\} \quad \text { and } \quad \mathcal{C}_{0}=\left\{\alpha=\left(\alpha_{0}, \alpha_{1}\right) \in \mathbb{Z}^{2 d} \backslash\{0\} \mid \alpha_{1}=0\right\}
$$

corresponding respectively to the waves propagating to the left and to the right, and to purely spatial oscillations. The reader should be cautious about the fact that the Fourier mode $\alpha=0$ is not considered as a part of this characteristic set. These sets essentially correspond to the characteristic variety of the linear Maxwell part of the equations. For coherences, we show the characteristic frequencies are those which resonate with some transition energy $\omega(m, n)$. They are given, for any wavenumber $k \in \mathbb{Z}^{d}$, by the resonant set

$$
\mathcal{R}(k)=\left\{\left(m, n, \alpha_{0}, \alpha_{1}\right) \in\{1, \ldots, N\}^{2} \times \mathbb{Z}^{2 d} \mid k \cdot \alpha_{1}=\omega(m, n)\right\} .
$$

Describing the asymptotic dynamics (2) - Rectification effects. The rectification phenomenon is the creation of non-oscillating terms through the nonlinear interaction of oscillating terms. This phenomenon is typical of quadratic systems such as (9).

It is the reason for our introduction of the intermediate time scale $T=t / \sqrt{\varepsilon}$ in (16). This scale captures the evolution of the system between the macroscopic length $|x|=\mathcal{O}(1)$ and the wavelength $\mathcal{O}(\varepsilon)$. The first need for the intermediate scale $T$ is, the non-oscillating terms induce a secular growth of the necessary corrector terms (see [31]). In that circumstance, it turns out that imposing a sublinearity condition,

$$
\frac{1}{T}\left\|\mathbf{U}^{j}\right\|_{L_{t, x, y, z, \sigma, \theta}^{\infty}} \underset{T \rightarrow+\infty}{\longrightarrow} 0 \text { for } j=1,2,
$$

ensures smallness of correctors in (13), namely $\sqrt{\varepsilon} \mathbf{U}^{j}(t, x, y, z, t / \sqrt{\varepsilon}, \sigma, \theta)=o(1)$ when $\varepsilon \rightarrow 0$ as desired $(j=1,2)$. A second reason for the introduction of the scale $T$ is that, already in the less 
singular regime of diffractive optics, the leading profile $\mathbf{U}^{0}$ obeys some linear, constant coefficients hyperbolic evolution system with respect to time $T$, and the $T$-sublinearity condition for correctors provides a unique way of determining $\mathbf{U}^{0}$ (see [26], [30], and [19], 20] for the variable coefficients case), via a detailed analysis of wave interactions at the scale $T$ (see Section 4.3). A similar phenomenon occurs in the present situation as well.

More precisely, the above mentioned system at scale $T$ here takes the following form. Separating the average part and the oscillating part as (we use the notation introduced in (16)-(18))

$$
\mathbf{u}^{0}=\mathbf{u}_{0}^{0}+\mathbf{u}_{\mathrm{osc}}^{0}, \quad \text { (which defines } \mathbf{u}_{\mathrm{osc}}^{0} \equiv \mathbf{u}^{0}-\mathbf{u}_{0}^{0} \text { ), }
$$

we prove that $\mathbf{u}_{0}^{0}$ satisfies a system of the form

$$
\partial_{T} \mathbf{u}_{0}^{0}+M_{2}\left(0, \partial_{y}, \partial_{z}\right) \mathbf{u}_{0}^{0}=0
$$

where $M_{2}\left(0, \partial_{y}, \partial_{z}\right)$ is a matrix-coefficient differential operator of size $3 \times 3$. Eventually, our analysis shows that (22) has to be solved together with the one giving the corrector $\mathbf{u}_{0}^{1}$, namely

$$
\partial_{T} \mathbf{u}_{0}^{1}+M_{2}\left(0, \partial_{y}, \partial_{z}\right) \mathbf{u}_{0}^{1}=-M_{1}\left(\partial_{t}, \partial_{x}\right) \mathbf{u}_{0}^{0},
$$

where $M_{1}\left(\partial_{t}, \partial_{x}\right)$ is another matrix differential operator. We establish that equation (23) possesses a unique solution $\mathbf{u}_{0}^{1}$ provided $T$-sublinearity of the right-hand-side is imposed. It turns out that the same "secular growth" analysis is also necessary for $\mathbf{u}_{\text {osc }}^{0}$ and $\mathbf{N}^{0}$.

Remark 2.1. In the particular case of profiles that do not depend on $T$, the coupled system (22), (23) is actually overdetermined (see Remark 6.1). Solvability is only recovered provided the matrix operators $M_{1}\left(\partial_{t}, \partial_{x}\right)$ and $M_{2}\left(0, \partial_{y}, \partial_{z}\right)$ commute. This very particular and important situation occurs in the Transverse Magnetic case (see below), where these operators are all scalar. In the general case, rectification enforces the introduction of time $T$ to make the set of profile equations solvable.

Describing the asymptotic dynamics (3) - the coherences. For coherences, we establish the relation

$$
\mathbf{C}^{0} \equiv \mathbf{C}^{0,1} e^{-\sigma}=\left(\sum_{\alpha \in \mathbb{Z}^{2 d}} \mathbf{C}_{\alpha}^{0,1} e^{i \alpha \cdot \theta}\right) e^{-\sigma}
$$

In other words, at dominant order coherences decay as $\exp (-\gamma t / \varepsilon)$. Besides, the coefficients $\mathbf{C}_{\alpha}^{0,1}$ are shown to satisfy (see (46))

$$
\forall(m, n, \alpha) \in \mathcal{R}(k), \quad \partial_{T} \mathbf{C}_{m, n, \alpha}^{0,1}=i\left[E^{0} \cdot \Gamma, \mathbf{C}^{0,1}\right]_{m, n, \alpha}
$$

The set $\mathcal{R}(k)$ is defined in (20). The other components $\mathbf{C}_{m, n, \alpha}^{0,1}$, for which $(m, n, \alpha) \notin \mathcal{R}(k)$, are shown to vanish (and $E^{0}$ is determined independently: see the paragraph "Field dynamics" below).

Now the resolution of (24) cannot rely on the above mentioned sublinearity condition, due to the fact that a generic solution to (24) grows like $\exp (K T)$ or so at least, for some constant $K>0$. Similarly, the analogous equations on the correctors $\mathbf{C}^{1}$ and $\mathbf{C}^{2}$ cannot be solved using the sublinearity condition neither. As a consequence, the evolution of $\mathbf{C}^{0}$ remains a priori undetermined. The key point now comes from the off-diagonal relaxations. Indeed, we do prove that $\mathbf{C}^{0,1}$ satisfies a bound of the form

$$
\forall \mu \in \mathbb{N}^{5+2 d}, \quad\left|\partial_{t, T, x, y, z, \theta}^{\mu} \mathbf{C}^{0,1}(T)\right| \leq K_{1} e^{K_{2} T},
$$


for some $K_{1}, K_{2}>0$, so that the product $\left.\left(\mathbf{C}^{0,1} \exp (-\sigma)\right)\right|_{T=t / \sqrt{\varepsilon}, \sigma=-\gamma t / \varepsilon}$ has size $\mathcal{O}\left(\exp \left(K_{2} t / \sqrt{\varepsilon}\right) \times\right.$ $\times \exp (-\gamma t / \varepsilon))$, hence is negligible when $\varepsilon \rightarrow 0$, as desired. We notice a posteriori that the lack of knowledge in the evolution of $\mathbf{C}^{0}$ is harmless, since coherences live during an initial layer of size $\mathcal{O}(\varepsilon)$ only. We also show that the correctors $\mathbf{C}^{1}$ and $\mathbf{C}^{2}$ are negligible thanks to estimates in the same vein (see Section 4.3.2).

Describing the asymptotic dynamics (4) - the electromagnetic field. First, we show that $\mathbf{u}^{0} \equiv \mathbf{u}^{0,0}$, so that the electromagnetic field does not undergo the same decay as coherences. Next, we prove that $\mathbf{u}^{0}$, conveniently decomposed into its average and its oscillatory part, may be written

$$
\mathbf{u}^{0}=\underbrace{\mathbf{u}_{0,0}^{0}+\mathbf{u}_{0,+}^{0}+\mathbf{u}_{0,-}^{0}}_{=\mathbf{u}_{0}^{0}}+\underbrace{\mathbf{u}_{\mathrm{time}}^{0}+\mathbf{u}_{\mathrm{space}}^{0}}_{=\mathbf{u}_{\mathrm{osc}}^{0}},
$$

where we define the purely spatial oscillations, and temporal oscillationsฐ, as

$$
\mathbf{u}_{\mathrm{space}}^{0}=\sum_{\alpha \in \mathcal{C}_{0}} \mathbf{u}_{\alpha}^{0} e^{i \alpha \cdot \theta}, \quad \mathbf{u}_{\mathrm{time}}^{0}=\sum_{\alpha \in \mathcal{C}_{+} \cup \mathcal{C}_{-}} \mathbf{u}_{\alpha}^{0} e^{i \alpha \cdot \theta}
$$

The three mean terms $\mathbf{u}_{0,0}^{0}, \mathbf{u}_{0,+}^{0}$ and $\mathbf{u}_{0,-}^{0}$ are defined in the course of the analysis. The above decomposition entails the fact that we have $\mathbf{u}_{\alpha}^{0}=0$ whenever $\alpha \notin \mathcal{C}_{0} \cup \mathcal{C}_{+} \cup \mathcal{C}_{-}$. This is a polarization condition, as usual in optics. Besides, there are algebraic projectors $\pi_{0}, \pi_{+}$and $\pi_{-}$, such that for any $\alpha \in \mathcal{C}_{\iota}$, we have $\pi_{\iota} \mathbf{u}_{\alpha}^{0}=\mathbf{u}_{\alpha}^{0}(\iota=0,+,-)$.

We also show that functions $\mathbf{u}_{0,0}^{0}, \mathbf{u}_{\text {space }}^{0}, \mathbf{u}_{0,+}^{0}, \mathbf{u}_{0,-}^{0}$ do not depend on the slow time $t$, i.e.

$$
\partial_{t} \mathbf{u}_{0,0}^{0}=0, \quad \mathbf{u}_{\text {space }}^{0}=0, \quad \partial_{t} \mathbf{u}_{0, \pm}^{0}=0,
$$

and they satisfy, from (22),

$$
\partial_{T} \mathbf{u}_{0,0}^{0}=0, \quad \partial_{T} \mathbf{u}_{\text {space }}^{0}=0, \quad\left(\partial_{T} \pm \sqrt{\mathrm{D}_{y}^{2}+\mathrm{D}_{z}^{2}}\right) \mathbf{u}_{0, \pm}^{0}=0 .
$$

The latter is a two-dimensional (non local) transport equation at time scale $T$. The time oscillations $\mathbf{u}_{\text {time }}^{0}$ satisfy

$$
\begin{aligned}
& \forall \alpha \in \mathcal{C}_{ \pm}, \quad \partial_{T} \mathbf{u}_{\alpha}^{0}=0, \quad \text { and } \\
& \qquad\left(\partial_{t} \pm \partial_{x}\right) \mathbf{u}_{\alpha}^{0} \mp \frac{i}{2 k \cdot \alpha_{0}} \Delta_{y, z} \mathbf{u}_{\alpha}^{0}=\pi_{ \pm}\left(0, i \operatorname{Tr}\left(\Gamma\left(\Omega_{\gamma}\left(\Omega_{\gamma}-k \cdot \alpha_{1}\right)^{-1}-1\right)\left[E_{\text {time }}^{0} \cdot \Gamma, \mathbf{N}^{0}\right]_{\alpha}\right)\right)
\end{aligned}
$$

a nonlinear Schrödinger equation with respect to time $t$, describing diffraction in the transverse variable $(y, z)$, and quadratic coupling between light and matter. Technically, a standard Diophantine hypothesis ensures that $k \cdot \alpha_{0}$ is "not to small" in (29) - see Hypothesis 3.3.

Describing the asymptotic dynamics (5) - the populations. As for the electromagnetic field, the dominant term $\mathbf{N}^{0} \equiv \mathbf{N}^{0,0}$ in the populations does not decay in time at first order. In addition, we show that $\mathbf{N}^{0}$ only possesses spatial oscillations, thanks to the identity

$$
\mathbf{N}^{0}=\mathbf{N}_{0}^{0}+\mathbf{N}_{\text {space }}^{0}
$$

\footnotetext{
${ }^{1}$ to be accurate, these oscillations, namely $\mathbf{u}_{\text {time }}^{0}$, involve both time and space variables - we neertheless keep the denomination "time" for this part of the oscillations.
} 
This is a polarization property. Furthermore, $\mathbf{N}^{0}$ does not depend on the intermediate time $T$,

$$
\partial_{T} \mathbf{N}^{0}=0
$$

Lastly, at the macroscopic time scale $t$, populations evolve according to a Boltzmann-type equation, with transition rates that are the sum of the linear rates $W$ (see (5)), and of nonlinear rates that depend quadratically on $E^{0}$ and change with the frequency $\theta$. Precisely, we get the relation

$$
\begin{aligned}
\partial_{t} \mathbf{N}^{0}=W \sharp \mathbf{N}^{0}- & {\left[\left(E_{\mathrm{osc}}^{0}+E_{0,0}^{0}\right) \cdot \Gamma,\left(i \Omega_{\gamma}-k \cdot \partial_{\theta_{1}}\right)^{-1}\left[\left(E_{\mathrm{osc}}^{0}+E_{0,0}^{0}\right) \cdot \Gamma, \mathbf{N}^{0}\right]_{\mathrm{od}}\right]_{\mathrm{d}, \mathrm{space}} } \\
- & {\left[\left(E_{\mathrm{osc}}^{0}+E_{0,0}^{0}\right) \cdot \Gamma,\left(i \Omega_{\gamma}-k \cdot \partial_{\theta_{1}}\right)^{-1}\left[\left(E_{\mathrm{osc}}^{0}+E_{0,0}^{0}\right) \cdot \Gamma, \mathbf{N}^{0}\right]_{\mathrm{od}}\right]_{\mathrm{d}, 0} . }
\end{aligned}
$$

We refer to (69) below. In practice, this equation is of the form

$$
\partial_{t} \mathbf{N}_{\alpha}^{0}=W \sharp \mathbf{N}_{\alpha}^{0}+\sum_{\alpha^{\prime}+\alpha^{\prime \prime}=\alpha} \sum_{\beta} W\left(\alpha^{\prime}, \alpha^{\prime \prime}, E_{\alpha^{\prime}}^{0}, E_{\alpha^{\prime \prime}-\beta}^{0}\right) \sharp \mathbf{N}_{\beta}^{0},
$$

for some Pauli coefficients $W\left(\alpha^{\prime}, \alpha^{\prime \prime}, E_{\alpha^{\prime}}^{0}, E_{\alpha^{\prime \prime}-\beta}^{0}\right)$ that depend on the Fourier indices $\alpha^{\prime} \in \mathbb{Z}^{2 d}, \alpha^{\prime \prime} \in$ $\mathbb{Z}^{2 d}, \beta \in \mathbb{Z}^{2 d}$, as well as linearly on each variable $E_{\alpha^{\prime}}^{0}$ and $E_{\alpha^{\prime \prime}-\beta}^{0}$.

An illustrative example : the Transverse Magnetic (TM) case. We detail here the above analysis in the classical TM case (see 34]), for which formulae become more explicit, and the geometry of the problem is a bit simpler. The TM case is a 2-dimensional case, for which in addition fields have the particular polarization

$$
B=B(t, x, y)=\left(\begin{array}{c}
B_{x} \\
B_{y} \\
0
\end{array}\right), \quad E=E(t, x, y)=\left(\begin{array}{c}
0 \\
0 \\
E
\end{array}\right)
$$

Firstly, we give the explicit profile equations in this specific situation. Secondly, we show that the introduction of the intermediate time $T$ is not necessary in that case (see Remark 2.1). Finally, we perform the analysis for prepared data, i.e. when coherences vanish at leading order. Eventually, we show that the approximation is better than in (15), in that Theorem 6.3 asserts

$$
\forall \mu \in \mathbb{N}^{2}, \quad\left\|\partial_{x, y}^{\mu}\left(\mathbf{U}^{\varepsilon}-\mathbf{U}_{\text {app }}^{\varepsilon}\right)\right\|_{L^{\infty}\left(\left[0, t_{\star}\right] \times \mathbb{R}^{2}\right)}=\mathcal{O}(\sqrt{\varepsilon}) .
$$

\subsection{Outline of the paper}

In Section 3, we describe the Ansatz (13), the choice of scales and phases. We also discuss the needed

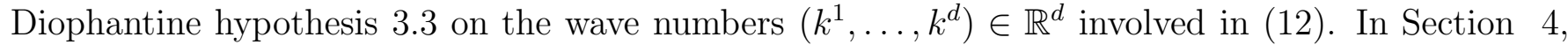
we proceed with the construction of an approximate solution $\mathbf{U}_{\text {app }}^{\varepsilon}=\mathbf{U}_{\text {app }}^{\varepsilon}(t, x, y, z, T, \theta)$ that is consistant with Maxwell-Bloch's system (9). Sections 4.1 and 4.2 contain the fast scale analysis, i.e. the analysis in the variable $\theta$. This is a Fourier analysis, leading to the usual characteristic sets and group velocity of geometric optics. Section 4.3 is devoted to the intermediate scale analysis of the resulting profile equations at the time scale $T$. The sublinearity condition (21) is involved (in Section 4.3.1), that allows to treat wave interactions. We also perform (in Section 4.3.2) the ad hoc analysis for the coherences $\mathbf{C}_{\mathrm{app}}^{\varepsilon}$, which have exponential growth in $T$ and violate the sublinearity condition. In Section 4.4, we eventually solve the Cauchy problem for profile equations in all variables $t, T$, and $\theta$. To complete the analysis, Section 5 provides the proof of our main result, namely that 
$\mathbf{U}_{\text {app }}^{\varepsilon}$ indeed approximates the true solution $\mathbf{U}^{\varepsilon}$ to the Maxwell-Bloch system (9), see equation (15) above. The precise form of our result is given in Theorem 5.2. Lastly, Section 6 goes again through the whole analysis, yet in the simpler Transverse Magnetic case, and for prepared initial data. The corresponding result is given in Theorem 6.3.

Our main theorems are Theorem 5.2 and Theorem 6.3 .

\section{Formulating the Ansatz}

We solve the Cauchy problem associated with (9) (with unknown $\mathbf{U}^{\varepsilon}=\left(\mathbf{u}^{\varepsilon}, \mathbf{C}^{\varepsilon}, \mathbf{N}^{\varepsilon}\right), \mathbf{u}^{\varepsilon}=\left(B^{\varepsilon}, E^{\varepsilon}\right)$ ) for rapidly oscillating initial data of the form

$$
\mathbf{U}_{\mathrm{ini}}^{\varepsilon}(x, y, z)=\sum_{\beta \in \mathbb{Z}^{d}} \phi_{\beta}^{\varepsilon}(x, y, z) \exp \left(i(\beta \cdot k) \frac{x}{\varepsilon}\right),
$$

where the wave vector $k$ is $k=\left(k^{1}, \ldots, k^{d}\right) \in \mathbb{R}^{d}$ for some $d \in \mathbb{N}^{\star}$, its coordinates are chosen $\mathbb{Q}$ independent, and the scalar product $\beta \cdot k$ in (32) denotes $\beta \cdot k=\beta^{1} k^{1}+\cdots+\beta^{d} k^{d}$. The $\mathbb{Q}$-independence of $k$ 's coordinates ensures that for $\beta \in \mathbb{Z}^{d}$, relation $\beta \cdot k=0$ holds if and only if $\beta=0$. The vector $k$ collects the independent oscillations (in $x$ ) carried by the initial signal $\left(B^{\varepsilon}, E^{\varepsilon}, \rho^{\varepsilon}\right)_{\text {ini }}(x, y, z)$, while the integer $\beta^{j} \in \mathbb{Z}$ numbers the various harmonics corresponding to the phase $k^{j} x / \varepsilon(j=1, \ldots, d)$. We choose an initial signal that carries all possible harmonics. This harmless choice is motivated by the fact that the original, nonlinear, Maxwell-Bloch system anyhow generates nonlinear wave interaction, which implies that any initial oscillation creates the associated harmonics along wave propagation.

The off-diagonal relaxation term $-\gamma \mathbf{C}^{\varepsilon} / \varepsilon$ in (9) enforces exponential decay of coherences $\mathbf{C}^{\varepsilon}$, so that, at first order, we expect only the fields $\mathbf{u}^{\varepsilon}=\left(B^{\varepsilon}, E^{\varepsilon}\right)$ and the populations $\mathbf{N}^{\varepsilon}$ to propagate. Hence space and time oscillations are expected to be generated from the initial spatial ones only through Maxwell's equations and through the equation for the populations. In a similar spirit, since our scaling postulates that variations in the transverse variables $(y, z)$ only occur at the scale $\sqrt{\varepsilon}$ (and not $\varepsilon$ ), we also expect that oscillations will not occur in the $(y, z)$ variables. In other words, the relevant Maxwell equations for oscillations are expected to be 1D in the $x$ direction. The corresponding characteristic variety, in Fourier variables, reads

$$
\mathcal{C}_{\text {Maxwell }} \cup \mathcal{C}_{\text {populations }}=\left\{(\tau, \xi) \in \mathbb{R}^{2} \backslash\{0\} \mid \tau=0 \text { or } \tau^{2}=\xi^{2}\right\}
$$

Eventually, propagation of space and time oscillations are expected to occur via the collection of phases

$$
k^{j} x-\omega^{j} t, \quad \text { with } \omega^{j}=0 \text { or } \omega^{j}= \pm k^{j} \quad(j=1, \ldots, d) .
$$

More precisely, the relevant oscillations in our analysis are expected to be all the harmonics $\beta \cdot(k x-$ $\omega t$ ), as the multi-index $\beta$ runs in $\mathbb{Z}^{d}$, and the $j$-th coordinate of $\omega$ is either $0, k^{j}$ or $-k^{j}$. An instant of reflexion shows that this ensemble coincides with the collection of phases $\left(\alpha_{1} \cdot k\right) x-\left(\alpha_{0} \cdot k\right) t$ as $\alpha_{0}$ and $\alpha_{1}$ run in $\mathbb{Z}^{d}$. This motivates the following

Notation 3.1. For any multi-index $\alpha=\left(\alpha^{1}, \ldots, \alpha^{2 d}\right) \in \mathbb{Z}^{2 d}$, we decompose

$$
\alpha:=\left(\alpha_{0}, \alpha_{1}\right), \text { with } \alpha_{0}=\left(\alpha^{1}, \ldots, \alpha^{d}\right) \in \mathbb{Z}^{d} \text {, and } \alpha_{1}=\left(\alpha^{d+1}, \ldots, \alpha^{2 d}\right) \in \mathbb{Z}^{d}
$$


Accordingly, any (smooth enough) function $\mathbf{V}(\theta)$ defined over the torus $\mathbb{T}^{2 d}$ may be decomposed in Fourier series as

$$
\mathbf{V}(\theta)=\sum_{\alpha \in \mathbb{Z}^{2 d}} \mathbf{V}_{\alpha} \exp (i \alpha \cdot \theta)=\sum_{\alpha \in \mathbb{Z}^{2 d}} \mathbf{V}_{\alpha} \exp \left(i\left(\alpha_{0} \cdot \theta_{0}+\alpha_{1} \cdot \theta_{1}\right)\right)
$$

With this notation, resonances are identified by the characteristic set $\mathcal{C}=\mathcal{C}_{0} \cup \mathcal{C}_{+} \cup \mathcal{C}_{-}$, where $\mathcal{C}_{0}, \mathcal{C}_{+}$, and $\mathcal{C}_{-}$have been defined in (19).

Due to the fast decay of coherences, another set of phases needs to be introduced. Namely, the off-diagonal relaxation term $-\gamma \mathbf{C}^{\varepsilon} / \varepsilon$ in (9) leads to introduce the complex phases $\exp (-\gamma t / \varepsilon)$ as well as all its harmonics $\exp (-\kappa \gamma t / \varepsilon)$, where $\kappa \in \mathbb{N}$.

With all these considerations in mind, we can now define the approximate solution we seek, as

$$
\begin{aligned}
& \mathbf{U}_{\text {app }}^{\varepsilon}=\left(\mathbf{u}_{\text {app }}^{\varepsilon}, \mathbf{C}_{\text {app }}^{\varepsilon}, \mathbf{N}_{\text {app }}^{\varepsilon}\right), \quad \mathbf{u}_{\text {app }}^{\varepsilon}=\left(B_{\text {app }}^{\varepsilon}, E_{\text {app }}^{\varepsilon}\right), \quad \text { where } \\
& \mathbf{U}_{\text {app }}^{\varepsilon}(t, x, y, z)=\left.\sum_{j=0}^{2} \sqrt{\varepsilon}^{j} \mathbf{U}^{j}\left(t, x, y, z, T, \sigma, \theta_{0}, \theta_{1}\right)\right|_{T=t / \sqrt{\varepsilon}, \sigma=\gamma t / \varepsilon,\left(\theta_{0}, \theta_{1}\right)=(k x,-k t) / \varepsilon},
\end{aligned}
$$

and for any $j=0,1,2$, and any $\sigma \geq 0, T \geq 0, \theta \in \mathbb{T}^{2 d}$, we set

$$
\mathbf{U}^{j}(t, x, y, z, T, \sigma, \theta)=\sum_{\alpha \in \mathbb{Z}^{2 d}} \sum_{\kappa \in \mathbb{N}} \mathbf{U}_{\alpha}^{j, \kappa}(t, x, y, z, T) \exp (i \alpha \cdot \theta) \exp (-\kappa \sigma) .
$$

\section{Remark 3.2. (discussion of the chosen profiles).}

(i) Coming back to the original scales of the problem, we may represent the solution $\mathbf{U}^{\varepsilon}$ under the form

$$
\mathbf{U}^{\varepsilon}(t, x, y, z)=\tilde{\mathbf{U}}^{\varepsilon}(t, x, \sqrt{\varepsilon} y, \sqrt{\varepsilon} z),
$$

where the function $\tilde{\mathbf{U}}^{\varepsilon}$ has variations in $\tilde{y}=\sqrt{\varepsilon} y$ and $\tilde{z}=\sqrt{\varepsilon} z$ at scale $\sqrt{\varepsilon}$, and solves (9) with the operator $\operatorname{curl}_{\varepsilon}$ replaced with curl, while the associated initial datum is of the form

$$
\tilde{\mathbf{U}}_{\left.\right|_{t=0} ^{\varepsilon}}^{\varepsilon}(x, \tilde{y}, \tilde{z})=\mathbf{U}_{\left.\right|_{t=0} ^{\varepsilon}}\left(x, \frac{\tilde{y}}{\sqrt{\varepsilon}}, \frac{\tilde{z}}{\sqrt{\varepsilon}}\right)=: \mathcal{U}_{\left.\right|_{t=0} ^{\varepsilon}}\left(x, \frac{\tilde{y}}{\sqrt{\varepsilon}}, \frac{\tilde{z}}{\sqrt{\varepsilon}}, \frac{x}{\varepsilon}\right) .
$$

This is the 3-scales setting of [20] (where only the case of quasilinear, non-dispersive systems is adressed). It leads to a profile representation of the form

$$
\tilde{\mathbf{U}}^{\varepsilon}(t, x, \tilde{y}, \tilde{z})=\tilde{\mathcal{U}}^{\varepsilon}\left(t, x, \tilde{y}, \tilde{z}, \frac{\psi}{\sqrt{\varepsilon}}, \frac{\phi}{\varepsilon}\right) .
$$

where $\phi=\phi(t, x, \tilde{y}, \tilde{z})$ is the collection of the two phases $k x$ and $-k t$, while $\psi=\psi(t, x, \tilde{y}, \tilde{z})$ is a collection of "intermediate phases" (in (33), $\tilde{\mathcal{U}}^{\varepsilon}$ does not depend explicitly on $\tilde{y}$, $\tilde{z}$, so that $\psi=$ $(t, \tilde{y}, \tilde{z}))$. When non-oscillating terms are present at first order $\left(\tilde{\mathbf{U}}_{0}^{0} \neq 0\right)$, it is in general necessary to put in this collection $\psi$ an intermediate time $t$ in order to solve the profile equations via the analysis of Section 4.3. This is the reason why the variable $T=t / \sqrt{\varepsilon}$ is present in (33) (it may be unnecessary: see [20], Remark 1.4, and the Transverse Magnetic case below, Section [0, for which $\psi=\tilde{y}$ ). This intermediate time $T$ captures the evolution of intermediate scales $\sqrt{\varepsilon}$, between "macroscopic length" $\mathcal{O}(1)$ and wavelength $\varepsilon$.

(ii) The consistancy of the chosen Ansatz requires that $\sqrt{\varepsilon} \mathbf{U}^{1} \ll \mathbf{U}^{0}$ whenever $\sigma=\gamma t / \varepsilon, T=t / \sqrt{\varepsilon}$, $\theta=(k x / \varepsilon,-k t / \varepsilon)$. This requirement enforces $T$-sublinearity of the non-exponentially decaying part 
(corresponding to $\kappa=0$ ) of the correctors $\mathbf{U}^{j, \kappa}(j \geq 1)$, see (61). The exponentially decaying correctors $(\kappa \geq 1)$ may be of the same order as the first profile $\mathbf{U}^{0, \kappa}$ : they anyhow lead to o $(\sqrt{\varepsilon})$ error terms, see Section 4.3.9.

(iii) The analogy with [20] shows that we could treat the same problem with curved (i.e. nonlinear) phases $\phi$. This situation arises in particular when dealing with inhomogeneous media, where variable coefficients (i.e. variable electric and magnetic permittivity) are involved in the Maxwell-Bloch system.

(iv) We emphasize the fact that in the present work, we deal with large population variations, of order one.

In 281 (long time diffraction, p. 248), with no relaxation terms, transparency allows to transform the original Maxwell-Bloch system, via a change of dependent variables, into a dispersive and quadratic system of the form $L(\varepsilon \partial) U=\varepsilon^{2} f(U)$, with $U=\left(U^{I}, U^{I I}\right)$, where $(B, E, \mathbf{C})=\varepsilon U^{I}$, and $\mathbf{N}=\varepsilon^{2} U^{I I}$. The system is considered over times of order $1 / \varepsilon$. Taking relaxations into account, we consider here a similar system, at the same space and time scales (oscillations have frequency $1 / \varepsilon$, and propagation is considered over times of order $1 / \varepsilon)$, yet in a situation where $(B, E, \mathbf{N}, \mathbf{C})$ are larger, of order 1, and the quadratic coupling term $f(U)$ is stronger, of size $\varepsilon$, namely

$$
\left\{\begin{array}{l}
\varepsilon \partial_{t} \mathbf{C}^{\varepsilon}=-i\left[\Omega, \mathbf{C}^{\varepsilon}\right]_{\mathrm{od}}+i \varepsilon\left[E^{\varepsilon} \cdot \Gamma, \mathbf{C}^{\varepsilon}\right]_{\mathrm{od}}+i \varepsilon\left[E^{\varepsilon} \cdot \Gamma, \mathbf{N}^{\varepsilon}\right]_{\mathrm{od}}-\gamma \mathbf{C}^{\varepsilon}, \\
\varepsilon \partial_{t} \mathbf{N}^{\varepsilon}=i \varepsilon\left[E^{\varepsilon} \cdot \Gamma, \mathbf{C}^{\varepsilon}\right]_{\mathrm{d}}+\varepsilon^{2} W \sharp \mathbf{N}^{\varepsilon} .
\end{array}\right.
$$

Of course, the stronger quadratic interaction term is balanced by the off-diagonal relaxations: note however that relaxation only affects part of the unknowns (the coherences), and one key aspect of our analysis precisely relies in the analysis of the stronger interaction term.

Such large population variations are also considered in [9], without relaxation, yet for waves that are polarized in a specific way, and in the case of a three-level Bloch system only (i.e. $N=3$ in our notation). In that case, additional conservation properties are at hand. On top of that, [9] considers a weaker coupling term of size $\varepsilon^{2}$. The limiting system obtained in [9] is of Schrödinger-Bloch type.

Since small divisor estimates naturally enter the analysis below, we readily formulate the usual Diophantine assumption we shall need on the wave-vector $k$. It will be used in order to invert the differential operators acting in the $\theta$ variable on the various (smooth) profiles $\mathbf{U}^{j, \kappa}(t, x, y, z, T, \theta)$. We refer for instance to [24].

Hypothesis 3.3. The wave vector $k \in \mathbb{R}^{s}$ from (32) is Diophantine, namely

$$
\exists C, a>0, \quad \forall \beta \in \mathbb{Z}^{d} \backslash\{0\}, \quad|\beta \cdot k| \geq C|\beta|^{-a} .
$$

Remark 3.4. The above assumption is harmless. Indeed, the following fact is well-known. Pick any exponent $a>d-1$. Then, the set

$$
\left\{\left.k \in \mathbb{R}^{d}\left|\exists C>0, \quad \forall \beta \in \mathbb{Z}^{d} \backslash\{0\}, \quad\right| \beta \cdot k|\geq C| \beta\right|^{-a}\right\}
$$

has full measure in $\mathbb{R}^{d}$. In other words, almost any $k \in \mathbb{R}^{d}$ (for the Lebesgue measure) has the Diophantine property.

\section{Formal expansions and approximate solution}

Notation 4.1. Denote by $M\left(\partial_{t}, \partial_{x}, \partial_{y}, \partial_{z}\right)$ the order 1 differential operator in Maxwell's equations,

$$
M\left(\partial_{t}, \partial_{x}, \partial_{y}, \partial_{z}\right)=\left(\begin{array}{cc}
0 & \operatorname{curl} \\
-\operatorname{curl} & 0
\end{array}\right)=\partial_{t}+A_{x} \partial_{x}+A_{y} \partial_{y}+A_{z} \partial_{z}
$$


where the $A_{j}$ 's are $6 \times 6$ real symmetric matrices. Set also

$$
M_{1}\left(\partial_{t}, \partial_{x}\right)=M\left(\partial_{t}, \partial_{x}, 0,0\right)=\partial_{t}+A_{x} \partial_{x}, \quad M_{2}\left(\partial_{T}, \partial_{y}, \partial_{z}\right)=M\left(\partial_{T}, 0, \partial_{y}, \partial_{z}\right)=\partial_{T}+A_{y} \partial_{y}+A_{z} \partial_{z}
$$

With this notation at hand, plugging the Ansatz (33) into the Maxwell-Bloch system (9), we get that $\mathbf{U}_{\text {app }}^{\varepsilon}$ satisfies the original Maxwell-Bloch equations up to a residual $r^{\varepsilon}$ that is given as follows (here we use the notation (11) for $\Omega_{\gamma}$ )

Proposition 4.2. The residual

$$
r^{\varepsilon}:=L\left(\partial_{t}, \partial_{x}, \frac{1}{\sqrt{\varepsilon}} \partial_{y}, \frac{1}{\sqrt{\varepsilon}} \partial_{z}\right) \mathbf{U}_{\mathrm{app}}^{\varepsilon}-F^{\varepsilon}\left(\mathbf{U}_{\mathrm{app}}^{\varepsilon}\right)
$$

has the profile representation

$$
r^{\varepsilon}(t, x, y, z)=\left.\mathcal{R}^{\varepsilon}(t, x, y, z, T, \sigma, \theta)\right|_{T=t / \sqrt{\varepsilon}, \sigma=\gamma t / \varepsilon,\left(\theta_{0}, \theta_{1}\right)=(k x,-k t) / \varepsilon},
$$

where

$$
\mathcal{R}^{\varepsilon}=\sum_{j=-2}^{3} \sum_{\kappa \geq 0} \sqrt{\varepsilon}^{j} r^{j, \kappa}(t, x, y, z, T, \theta) \exp (-\kappa \sigma),
$$

and the first terms $(-2 \leq j \leq 0)$ are (see Section 5.1 for the others)

$$
\begin{gathered}
r^{-2, \kappa}=\left(\begin{array}{c}
M_{1}\left(-\gamma \kappa-k \cdot \partial_{\theta_{1}}, k \cdot \partial_{\theta_{0}}\right) \mathbf{u}^{0, \kappa} \\
\left(i \Omega_{\gamma}-\gamma \kappa-k \cdot \partial_{\theta_{1}}\right) \mathbf{C}^{0, \kappa} \\
\left(-\gamma \kappa-k \cdot \partial_{\theta_{1}}\right) \mathbf{N}^{0, \kappa}
\end{array}\right), \\
r^{-1, \kappa}=\left(\begin{array}{l}
M_{1}\left(-\gamma \kappa-k \cdot \partial_{\theta_{1}}, k \cdot \partial_{\theta_{0}}\right) \mathbf{u}^{1, \kappa}+M_{2}\left(\partial_{T}, \partial_{y}, \partial_{z}\right) \mathbf{u}^{0, \kappa}-\left(0, i \operatorname{Tr}\left(\Gamma \Omega_{\gamma} \mathbf{C}^{0, \kappa}\right)\right) \\
\left(i \Omega_{\gamma}-\gamma \kappa-k \cdot \partial_{\theta_{1}}\right) \mathbf{C}^{1, \kappa}+\partial_{T} \mathbf{C}^{0, \kappa}-i\left[E^{0} \cdot \Gamma, \mathbf{C}^{0}+\mathbf{N}^{0}\right]_{\mathrm{od}}^{\kappa} \\
\left(-\gamma \kappa-k \cdot \partial_{\theta_{1}}\right) \mathbf{N}^{1, \kappa}+\partial_{T} \mathbf{N}^{0, \kappa}-i\left[E^{0} \cdot \Gamma, \mathbf{C}^{0}\right]_{\mathrm{d}}^{\kappa}
\end{array}\right), \\
r^{0, \kappa}=\left(\begin{array}{c}
M_{1}\left(-\gamma \kappa-k \cdot \partial_{\theta_{1}}, k \cdot \partial_{\theta_{0}}\right) \mathbf{u}^{2, \kappa}+M_{2}\left(\partial_{T}, \partial_{y}, \partial_{z}\right) \mathbf{u}^{1, \kappa} \\
+M_{1}\left(\partial_{t}, \partial_{x}\right) \mathbf{u}^{0, \kappa}+\left(0,-i \operatorname{Tr}\left(\Gamma \Omega_{\gamma} \mathbf{C}^{1, \kappa}\right)+i \operatorname{Tr}\left(\Gamma\left[E^{0} \cdot \Gamma, \mathbf{C}^{0}+\mathbf{N}^{0}\right]^{\kappa}\right)\right) \\
\left(i \Omega_{\gamma}-\gamma \kappa-k \cdot \partial_{\theta_{1}}\right) \mathbf{C}^{2, \kappa}+\partial_{T} \mathbf{C}^{1, \kappa}+\partial_{t} \mathbf{C}^{0, \kappa} \\
-i\left[E^{0} \cdot \Gamma, \mathbf{C}^{1}+\mathbf{N}^{1}\right]_{\mathrm{od}}^{\kappa}-i\left[E^{1} \cdot \Gamma, \mathbf{C}^{0}+\mathbf{N}^{0}\right]_{\mathrm{od}}^{\kappa} \\
\left(-\gamma \kappa-k \cdot \partial_{\theta_{1}}\right) \mathbf{N}^{2, \kappa}+\partial_{T} \mathbf{N}^{1, \kappa}+\partial_{t} \mathbf{N}^{0, \kappa}-i\left[E^{0} \cdot \Gamma, \mathbf{C}^{1}\right]_{\mathrm{d}}^{\kappa}-i\left[E^{1} \cdot \Gamma, \mathbf{C}^{0}\right]_{\mathrm{d}}^{\kappa}-W \sharp \mathbf{N}^{0, \kappa}
\end{array}\right) .
\end{gathered}
$$

With this computation at hand, we wish to construct the first profile $\mathbf{U}^{0, \kappa}=\left(\mathbf{u}^{0, \kappa}, \mathbf{C}^{0, \kappa}, \mathbf{N}^{0, \kappa}\right)$, in $\mathcal{C}^{0}\left(\left[0, t_{\star}\right]_{t}, \mathcal{C}^{0}\left(\left[0,+\infty\left[T, H^{\infty}\left(\mathbb{R}^{3} \times \mathbb{T}^{2 d}\right)\right)\right)\right.\right.$ for some $t_{\star}>0$, together with correctors $\left(\mathbf{u}^{j}, \mathbf{C}^{j}, \mathbf{N}^{j}\right)$ $(j=1,2)$, in such a way that the residual $r^{\varepsilon}$ is small (cf. Section 5.2). More precisely, we shall impose $r^{-2}=r^{-1}=r^{0}=0$, and show that this procedure completely determines $\mathbf{U}^{0}$. This is obtained by decomposing successively (with increasing $j$ ) the equations obtained for the $\mathbf{U}^{j}$ 's, and separating characteristic and noncharacteristic modes $(\alpha, \kappa)$. The necessary linear algebra tools are developed in the next paragraph. 


\subsection{Rapid modes and algebraic projectors}

- Tools needed to deal with the electromagnetic field. The Fourier series representation, in the $\theta$ variable, of the first equation stemming from $\left[r^{-2, \kappa}=0\right]$ is (according to notation 4.1)

$$
M_{1}\left(-\gamma \kappa-i k \cdot \alpha_{1}, i k \cdot \alpha_{0}\right) \mathbf{u}_{\alpha}^{0, \kappa}=0, \quad \forall \alpha \in \mathbb{Z}^{2 d} .
$$

For each $\alpha$, this is a system of linear equations in $\mathbb{C}^{6}$, with matrix

$$
M_{1}\left(-\gamma \kappa-i k \cdot \alpha_{1}, i k \cdot \alpha_{0}\right)=i k \cdot \alpha_{1}+i\left(k \cdot \alpha_{0}\right) A_{x}
$$

which may be singular only if $\kappa=0$. To deal with this equation, we need the following classical definitions and lemmas ([32], [24], [18, [26]).

Definition 4.3. For each $\alpha \in \mathbb{Z}^{2 d}$, let $\pi_{\alpha}$ be the orthogonal projection in $\mathbb{R}^{6}$ onto the kernel of $M_{1}\left(-i k \cdot \alpha_{1}, i k \cdot \alpha_{0}\right)$. Denote by $M_{1}\left(-i k \cdot \alpha_{1}, i k \cdot \alpha_{0}\right)^{-1}$ the inverse of $M_{1}\left(-i k \cdot \alpha_{1}, i k \cdot \alpha_{0}\right)$, when restricted to the space orthogonal to its kernel, namely $M_{1}\left(-i k \cdot \alpha_{1}, i k \cdot \alpha_{0}\right)^{-1}$ acts on $\operatorname{Ran}\left(1-\pi_{\alpha}\right)$. Finally, define the projector $\Pi$ on the space of Fourier series,

$$
\Pi\left(\sum_{\alpha \in \mathbb{Z}^{2 d}} u_{\alpha} e^{i \alpha \cdot \theta}\right):=\sum_{\alpha \in \mathbb{Z}^{2 d}} \pi_{\alpha} u_{\alpha} e^{i \alpha \cdot \theta}
$$

A straightforward computation establishes the

Lemma 4.4. For each $\alpha \in \mathbb{Z}^{2 d}$, the projector $\pi_{\alpha}$ is a homogeneous function of $\alpha$ of degree zero. It takes a constant (matrix) value $\pi^{\iota}$ on each component $\mathcal{C}_{\iota}$ of the characteristic set $(\iota=+,-, 0)$, the value one (or identity) for $\alpha=0$, and vanishes else. In particular, we have the identity

$$
\Pi\left(\sum_{\alpha \in \mathbb{Z}^{2 d}} u_{\alpha} e^{i \alpha \cdot \theta}\right):=u_{0}+\sum_{\alpha \in \mathcal{C}_{+}} \pi^{+} u_{\alpha} e^{i \alpha \cdot \theta}+\sum_{\alpha \in \mathcal{C}_{-}} \pi^{-} u_{\alpha} e^{i \alpha \cdot \theta}+\sum_{\alpha \in \mathcal{C}_{0}} \pi^{0} u_{\alpha} e^{i \alpha \cdot \theta} .
$$

In any circumstance, for any Fourier series $u=\sum_{\alpha \in \mathbb{Z}^{2 d}} u_{\alpha} e^{i \alpha \cdot \theta}$, we have

$$
M_{1}\left(-k \cdot \partial_{\theta_{1}}, k \cdot \partial_{\theta_{0}}\right) u=0 \Longleftrightarrow \Pi u=u \text {. }
$$

Next, the following two lemmas are classical for geometric and diffractive optics with smooth characteristic varieties ([32], [24], [18], and also [41] for an elegant unified version). They express that the operators acting on the oscillating part of the fields are in diagonal form.

Lemma 4.5 (group velocity).

For any Fourier series $u=\sum_{\alpha \in \mathbb{Z}^{2 d} \backslash\{0\}} u_{\alpha} e^{i \alpha \cdot \theta}$, containing no mean term, we have, with $\mathrm{v}(\alpha)=\iota$ for $\alpha \in \mathcal{C}_{\iota}(\iota=0,+,-)$, the relations

$$
\Pi A_{y} \Pi u=\Pi A_{z} \Pi u=0, \quad \text { and } \quad \Pi A_{x} \Pi u=\sum_{\alpha \in \mathcal{C}} \mathrm{v}(\alpha) \pi_{\alpha} u_{\alpha} e^{i \alpha \cdot \theta}=: \mathrm{v}\left(\mathrm{D}_{\theta}\right) \Pi u
$$


Lemma 4.6 (diffraction).

For any Fourier series $u=\sum_{\alpha \in \mathbb{Z}^{2 d} \backslash\{0\}} u_{\alpha} e^{i \alpha \cdot \theta}$, containing no mean term, we have

$$
\begin{array}{r}
\Pi M_{2}\left(0, \partial_{y}, \partial_{z}\right) M_{1}\left(-k \cdot \partial_{\theta_{1}}, k \cdot \partial_{\theta_{0}}\right)^{-1} M_{2}\left(0, \partial_{y}, \partial_{z}\right) \Pi u \\
=i \sum_{\alpha \in \mathcal{C}_{+} \cup \mathcal{C}_{-}} \mathrm{a}(\alpha) \Delta_{y, z} \pi_{\alpha} u_{\alpha} e^{i \alpha \cdot \theta}=: i \mathrm{a}\left(\mathrm{D}_{\theta}\right) \Delta_{y, z} \Pi u
\end{array}
$$

where $\mathrm{a}(\alpha)= \pm \frac{1}{2 k \cdot \alpha_{0}}$ for $\alpha \in \mathcal{C}_{ \pm}$and $\mathrm{a}(\alpha)=0$ for $\alpha \in \mathcal{C}_{0}$

Proof. By the definition of the operators involved, for each $\alpha$, one has

$$
\pi_{\alpha} M_{2}\left(0, \partial_{y}, \partial_{z}\right) M_{1}\left(-k \cdot \alpha_{1}, k \cdot \alpha_{0}\right)^{-1} M_{2}\left(0, \partial_{y}, \partial_{z}\right) \pi_{\alpha}=\sum_{j, k \in\{y, z\}} \pi_{\alpha} A_{j} M_{1}\left(-k \cdot \alpha_{1}, k \cdot \alpha_{0}\right)^{-1} A_{k} \pi_{\alpha} \partial_{j} \partial_{k} .
$$

Now, $\pi_{\alpha}$ and $M_{1}\left(-k \cdot \alpha_{1}, k \cdot \alpha_{0}\right)^{-1}$ are the evaluations, at $\xi=\left(k \cdot \alpha_{0}, 0,0\right)$, of the spectral projector $P(\tau(\xi), \xi)$ resp. of the pseudo-inverse $M^{-1}(\tau(\xi), \xi)$ of the complete Maxwell symbol $M(\tau(\xi), \xi)$. Here and in the sequel, we use the notation

$$
\tau_{ \pm}(\xi)=\mp|\xi| \quad \text { for } \quad \alpha \in \mathcal{C}_{\iota, \pm}:=\mathcal{C}_{\iota} \cap\left\{k \cdot \alpha_{1} \gtrless 0\right\}, \quad \iota= \pm
$$

together with

$$
\tau(\xi)=0 \text { for } \alpha \in \mathcal{C}_{0}
$$

The quantities $\tau_{ \pm}(\xi)$ and $\tau(\xi)$ are naturally the eigenvalues of the Maxwell symbol $M(\tau(\xi), \xi)$. On the other hand, we have the identity, valid for any $j, k \in\{x, y, z\}$, see [24] or [41],

$$
\begin{aligned}
& \frac{\partial^{2} \tau}{\partial_{\xi_{j}} \partial_{\xi_{k}}}(\xi) P(\tau(\xi), \xi) \\
& \quad=P(\tau(\xi), \xi) A_{j} M^{-1}(\tau(\xi), \xi) A_{k} P(\tau(\xi), \xi)+P(\tau(\xi), \xi) A_{k} M^{-1}(\tau(\xi), \xi) A_{j} P(\tau(\xi), \xi) .
\end{aligned}
$$

Using now the obvious relation

$$
\left(\frac{\partial^{2} \tau_{ \pm}}{\partial \xi_{j} \partial \xi_{k}}(\xi)\right)_{i, j}=\mp \frac{1}{|\xi|}\left(\mathrm{id}-\frac{\xi \otimes \xi}{|\xi|^{2}}\right)=\mp \frac{1}{\left|k \cdot \alpha_{0}\right|} \operatorname{Diag}(0,1,1) \quad \text { for } \xi=\left(k \cdot \alpha_{0}, 0,0\right)
$$

inspection of the five cases $\alpha \in \mathcal{C}_{\iota, \pm}(\iota= \pm)$, and $\alpha \in \mathcal{C}_{0}$, leads to the lemma.

Lastly, in order to distinguish between propagated and non-propagated parts of the profiles, we introduce the following splitting, refering to the "oscillating" part of the fields, to the "time" oscillations of the density matrix, or to its "space" oscillations.

Definition 4.7. For any Fourier series $U=\sum_{\alpha \in \mathbb{Z}^{2 d}} U_{\alpha} e^{i \alpha \cdot \theta}$, we set

$$
U_{\mathrm{osc}}:=\sum_{\alpha \neq 0} U_{\alpha} e^{i \alpha \cdot \theta}, \quad U_{\mathrm{space}}:=\sum_{\alpha \in \mathcal{C}_{0}} U_{\alpha} e^{i \alpha \cdot \theta}, \quad U_{\mathrm{time}}:=\sum_{\alpha \in \mathcal{C}_{+} \cup \mathcal{C}_{-}} U_{\alpha} e^{i \alpha \cdot \theta}
$$


- Tools needed to deal with the coherences. For each mode $(\alpha, \kappa)$, the second equation from $\left[r_{\alpha}^{-2, \kappa}=0\right]$ reads

$$
\forall m, n, \quad\left(i\left(\omega(m, n)-k \cdot \alpha_{1}\right)+\gamma(1-\kappa)\right) \mathbf{C}_{m, n, \alpha}^{0, \kappa}=0 .
$$

Here, only $\kappa=1$ is of interest. This justifies the introduction of the resonant set $\mathcal{R}(k)$ we defined in (20).

Remark 4.8. In [9], the wave vector $k$ is precisely chosen so that $\mathcal{R}(k)$ be nonempty.

- Tools needed to deal with the populations. The populations are scalar variables, and the characteristic frequencies are simply the $(\alpha, \kappa)$ 's belonging to $\mathcal{C}_{0} \times\{0\}$. No additional tool is needed.

\subsection{Profile equations, fast scale analysis}

\subsubsection{The residual $r^{-2}$}

According to the notation above, the equation $\left[r^{-2}=0\right]$ is equivalent to the polarization conditions

$$
\begin{array}{llll}
\mathbf{u}^{0}=\mathbf{u}^{0,0}, & \forall \kappa>0, & \mathbf{u}^{0, \kappa}=0, \quad \text { and } & \mathbf{u}^{0}=\Pi \mathbf{u}^{0}, \\
\mathbf{C}^{0}=\mathbf{C}^{0,1} e^{-\sigma}, & \text { and } & \forall(m, n, \alpha) \notin \mathcal{R}(k), & \mathbf{C}_{m, n, \alpha}^{0,1}=0, \\
\mathbf{N}^{0}=\mathbf{N}^{0,0}, & \text { and } \quad \mathbf{N}^{0}=\mathbf{N}_{0}^{0}+\mathbf{N}_{\text {space }}^{0} &
\end{array}
$$

In order not to overweight notation, we shall from now on systematically refer to $\mathbf{u}^{0}$ and $\mathbf{N}^{0}$ in the sequel, keeping in mind they do coincide with $\mathbf{u}^{0,0}$ and $\mathbf{N}^{0,0}$.

\subsubsection{The residual $r^{-1}$}

Equations on the field. We first deal with the mode $\kappa=0$. Separating the mean term $(\alpha=0)$ and the other Fourier modes, we find

$$
\begin{aligned}
& \partial_{T} \mathbf{u}_{0}^{0}+M_{2}\left(0, \partial_{y}, \partial_{z}\right) \mathbf{u}_{0}^{0}=0 \\
& \partial_{T} \mathbf{u}_{\mathrm{osc}}^{0}=0
\end{aligned}
$$

Obtaining the second equation requires to use (35) and Lemma 4.5, which imply $\Pi M_{2}\left(0, \partial_{y}, \partial_{z}\right) \Pi=0$ and $\mathbf{u}_{\mathrm{osc}}^{0}=\Pi \mathbf{u}_{\mathrm{osc}}^{0}$. The next order profile $\mathbf{u}^{1,0}$ is then seen to satisfy

$$
(1-\Pi) \mathbf{u}_{\mathrm{osc}}^{1,0}=-M_{1}\left(-k \cdot \partial_{\theta_{1}}, k \cdot \partial_{\theta_{0}}\right)^{-1} M_{2}\left(0, \partial_{y}, \partial_{z}\right) \mathbf{u}_{\mathrm{osc}}^{0} .
$$

Secondly, when $\kappa \geq 1$, the operator $M_{1}\left(-\gamma \kappa-k \cdot \partial_{\theta_{1}}, k \cdot \partial_{\theta_{0}}\right)$ is invertible, and we get the two values

$$
\begin{aligned}
& \mathbf{u}^{1,1}=M_{1}\left(-\gamma \kappa-k \cdot \partial_{\theta_{1}}, k \cdot \partial_{\theta_{0}}\right)^{-1}\left(0, i \operatorname{Tr}\left(\Gamma \Omega_{\gamma} \mathbf{C}^{0,1}\right)\right), \\
& \forall \kappa>1, \quad \mathbf{u}^{1, \kappa}=0 .
\end{aligned}
$$


Equations on the coherences. We first note that whenever $\kappa \neq 1$ the operator $\left(i \Omega_{\gamma}-\gamma \kappa-k \cdot \partial_{\theta_{0}}\right)$ is invertible. Thanks to (35), (36) and (37), this gives the two values

$$
\begin{aligned}
& \mathbf{C}^{1,0}=i\left(i \Omega_{\gamma}-k \cdot \partial_{\theta_{1}}\right)^{-1}\left[E^{0} \cdot \Gamma, \mathbf{N}^{0}\right]_{\mathrm{od}}, \\
& \forall \kappa>1, \quad \mathbf{C}^{1, \kappa}=0 .
\end{aligned}
$$

When $\kappa=1$, we need to distinguish between resonant and non-resonant triples $(m, n, \alpha)$, to obtain

$$
\begin{aligned}
& \forall(m, n, \alpha) \notin \mathcal{R}(k), \quad \mathbf{C}_{m, n, \alpha}^{1,1}=-\left(\omega(m, n)-k \cdot \alpha_{1}\right)^{-1}\left[E^{0} \cdot \Gamma, \mathbf{C}^{0,1}\right]_{m, n, \alpha} \\
& \forall(m, n, \alpha) \in \mathcal{R}(k), \quad \partial_{T} \mathbf{C}_{m, n, \alpha}^{0,1}=i\left[E^{0} \cdot \Gamma, \mathbf{C}^{0,1}\right]_{m, n, \alpha}
\end{aligned}
$$

Equations on the populations. Here we need to distinguish between the values $\kappa=0$ and $\kappa \neq 0$, as well as between the modes $\alpha \in \mathcal{C}_{0} \cup\{0\}$ and the other Fourier modes. In that way we obtain, taking $\kappa=0$ and restricting to modes $\alpha \in \mathcal{C}_{0} \cup\{0\}$, the relation

$$
\partial_{T} \mathbf{N}^{0}=0
$$

together with the following polarization conditions and spectral properties for $\mathbf{N}^{1}$ (here we use (37))

$$
\forall \alpha \notin \mathcal{C}_{0} \cup\{0\}, \mathbf{N}_{\alpha}^{1,0}=0, \quad \mathbf{N}^{1,1}=i\left(-\gamma-k \cdot \partial_{\theta_{1}}\right)^{-1}\left[E^{0} \cdot \Gamma, \mathbf{C}^{0,1}\right]_{\mathrm{d}}, \quad \forall \kappa>1, \mathbf{N}^{1, \kappa}=0 .
$$

\subsubsection{The residual $r^{0}$}

Equations on the field. When $\kappa=0$, we have (using (43) and (36))

$$
\begin{aligned}
0= & M_{1}\left(-k \cdot \partial_{\theta_{1}}, k \cdot \partial_{\theta_{0}}\right) \mathbf{u}^{2,0}+M_{2}\left(\partial_{T}, \partial_{y}, \partial_{z}\right) \mathbf{u}^{1,0}+M_{1}\left(\partial_{t}, \partial_{x}\right) \mathbf{u}^{0,0} \\
& +\left(0, i \operatorname{Tr}\left(\Gamma\left[E^{0,0} \cdot \Gamma, \mathbf{N}^{0,0}\right]\right)-i \operatorname{Tr}\left(\Gamma i \Omega_{\gamma}\left(i \Omega_{\gamma}-k \cdot \partial_{\theta_{1}}\right)^{-1}\left[E^{0,0} \cdot \Gamma, \mathbf{N}^{0,0}\right]_{\text {od }}\right)\right) .
\end{aligned}
$$

Again, we separate oscillating and nonoscillating parts in the above equation. In the nonoscillating case, the two nonlinear contributions are seen to compensate each other and we obtain

$$
M_{2}\left(\partial_{T}, \partial_{y}, \partial_{z}\right) \mathbf{u}_{0}^{1,0}=-M_{1}\left(\partial_{t}, \partial_{x}\right) \mathbf{u}_{0}^{0}
$$

On the other hand, the $\Pi$-polarized part of oscillating modes turns out to satisfy the relation, using (39), (40), (43) and Lemma 4.5, and writing $M_{2}\left(\partial_{T}, \partial_{y}, \partial_{z}\right)=\partial_{T}+M_{2}\left(0, \partial_{y}, \partial_{z}\right)$,

$$
\begin{aligned}
\partial_{T} \Pi \mathbf{u}_{\mathrm{osc}}^{1,0}=-\Pi M_{1}\left(\partial_{t}, \partial_{x}\right) \mathbf{u}_{\mathrm{osc}}^{0} & +\Pi M_{2}\left(0, \partial_{y}, \partial_{z}\right) M_{1}\left(-k \cdot \partial_{\theta_{1}}, k \cdot \partial_{\theta_{0}}\right)^{-1} M_{2}\left(0, \partial_{y}, \partial_{z}\right) \mathbf{u}_{\mathrm{osc}}^{0} \\
& +\Pi\left(0, i \operatorname{Tr}\left(\Gamma\left(i \Omega_{\gamma}\left(i \Omega_{\gamma}-k \cdot \partial_{\theta_{1}}\right)^{-1}-1\right)\left[E^{0} \cdot \Gamma, \mathbf{N}^{0}\right]_{\mathrm{osc}}\right)\right) .
\end{aligned}
$$

Thanks to Lemma 4.5 and Lemma 4.6, this is rewritten, using $\mathbf{u}_{\mathrm{osc}}^{0}=\Pi \mathbf{u}_{\mathrm{osc}}^{0}$,

$$
\begin{aligned}
\partial_{T} \Pi \mathbf{u}_{\mathrm{osc}}^{1,0}=- & \left(\partial_{t}+\mathrm{v}\left(\mathrm{D}_{\theta}\right)\right) \mathbf{u}_{\mathrm{osc}}^{0}+i \mathrm{a}\left(\mathrm{D}_{\theta}\right) \Delta_{y, z} \mathbf{u}_{\mathrm{osc}}^{0} \\
& +\Pi\left(0, i \operatorname{Tr}\left(\Gamma\left(i \Omega_{\gamma}\left(i \Omega_{\gamma}-k \cdot \partial_{\theta_{1}}\right)^{-1}-1\right)\left[E^{0} \cdot \Gamma, \mathbf{N}^{0}\right]_{\mathrm{osc}}\right)\right) .
\end{aligned}
$$

Lastly, applying $(1-\Pi)$ to the oscillating part of equation (49), we also recover (using the fact that $\partial_{T}\left((1-\Pi) \mathbf{u}_{\text {osc }}^{1,0}\right)=0$, deduced from equation (40) together with the identity $\left.\partial_{T} \mathbf{u}_{\text {osc }}^{0}=0\right)$,

$$
\begin{aligned}
(1-\Pi) \mathbf{u}_{\mathrm{osc}}^{2,0}= & -M_{1}\left(-k \cdot \partial_{\theta_{1}}, k \cdot \partial_{\theta_{0}}\right)^{-1}\left(M_{2}\left(0, \partial_{y}, \partial_{z}\right) \mathbf{u}_{\mathrm{osc}}^{1,0}+M_{1}\left(\partial_{t}, \partial_{x}\right) \mathbf{u}_{\mathrm{osc}}^{0}\right. \\
& \left.+\left(0, i \operatorname{Tr}\left(\Gamma\left(i \Omega_{\gamma}\left(i \Omega_{\gamma}-k \cdot \partial_{\theta_{1}}\right)^{-1}-1\right)\left[E^{0} \cdot \Gamma, \mathbf{N}^{0}\right]_{\mathrm{osc}}\right)\right)\right) .
\end{aligned}
$$


When $\kappa=1$, using (41), (35), (37), we get in the same way

$$
\begin{aligned}
\mathbf{u}^{2,1}=-M_{1}\left(-\gamma-k \cdot \partial_{\theta_{1}}, k \cdot \partial_{\theta_{0}}\right)^{-1} & {\left[M_{2}\left(\partial_{T}, \partial_{y}, \partial_{z}\right) M_{1}\left(-k \cdot \partial_{\theta_{1}}, k \cdot \partial_{\theta_{0}}\right)^{-1}\left(0, i \operatorname{Tr}\left(\Gamma\left(i \Omega_{\gamma} \mathbf{C}^{0,1}\right)\right)\right)\right.} \\
& \left.-\left(0, i \operatorname{Tr}\left(\Gamma \Omega_{\gamma} \mathbf{C}^{1,1}\right)\right)+i \operatorname{Tr}\left(\left[E^{0} \cdot \Gamma, \mathbf{C}^{0,1}\right]\right)\right] .
\end{aligned}
$$

Finally, for greater values of $\kappa$, using (42), (35), (44), (36), (37), we have

$$
\forall \kappa>1, \quad \mathbf{u}^{2, \kappa}=0 .
$$

Equations on the coherences. When $\kappa \neq 1$, we may invert directly

$$
\mathbf{C}^{2, \kappa}=-\left(i \Omega_{\gamma}-\gamma \kappa-k \cdot \partial_{\theta_{1}}\right)^{-1}\left(\partial_{T} \mathbf{C}^{1, \kappa}+\partial_{t} \mathbf{C}^{0, \kappa}-i\left[E^{0} \cdot \Gamma, \mathbf{C}^{1}+\mathbf{N}^{1}\right]_{\mathrm{od}}^{\kappa}-i\left[E^{1} \cdot \Gamma, \mathbf{C}^{0}+\mathbf{N}^{0}\right]_{\mathrm{od}}^{\kappa}\right),
$$

which vanishes for $\kappa>2$, thanks to (44), (36), (48), (37).

For $\kappa=1$, using (36), (37), (44), (48) and (42), nonresonant triples $(m, n, \alpha)$ lead to the similar formula,

$$
\begin{gathered}
\forall(m, n, \alpha) \notin \mathcal{R}(k), \quad \mathbf{C}_{m, n, \alpha}^{2,1}=- \\
\left(i \omega(m, n)+\gamma-i k \cdot \alpha_{1}\right)^{-1}\left(\partial_{T} \mathbf{C}_{m, n, \alpha}^{1,1}-i\left[E^{0} \cdot \Gamma, \mathbf{C}^{1,1}+\mathbf{N}^{1,1}\right]_{m, n, \alpha}\right. \\
\left.-i\left[E^{1,0} \cdot \Gamma, \mathbf{C}^{0,1}\right]_{m, n, \alpha}-i\left[E^{1,1} \cdot \Gamma, \mathbf{N}^{0}\right]_{m, n, \alpha}\right)
\end{gathered}
$$

whereas for resonant triples we obtain

$$
\begin{aligned}
\forall(m, n, \alpha) \in \mathcal{R}(k), \quad \partial_{T} \mathbf{C}_{m, n, \alpha}^{1,1}= & -\partial_{t} \mathbf{C}_{m, n, \alpha}^{0,1}+i\left[E^{0} \cdot \Gamma, \mathbf{C}^{1,1}+\mathbf{N}^{1,1}\right]_{m, n, \alpha} \\
& +i\left[E^{1,0} \cdot \Gamma, \mathbf{C}^{0,1}\right]_{m, n, \alpha}+i\left[E^{1,1} \cdot \Gamma, \mathbf{N}^{0}\right]_{m, n, \alpha}
\end{aligned}
$$

Equations on the population. For $\kappa=0$, using (35), (36), (37), (44) and (48), we have

$$
\begin{array}{ll}
\forall \alpha \in \mathcal{C}_{0} \cup\{0\}, & \partial_{T} \mathbf{N}_{\alpha}^{1,0}=-\partial_{t} \mathbf{N}_{\alpha}^{0}+i\left[E^{0} \cdot \Gamma, \mathbf{C}^{1,0}\right]_{\mathrm{d}, \alpha}+W \sharp \mathbf{N}_{\alpha}^{0}, \\
\forall \alpha \notin \mathcal{C}_{0} \cup\{0\}, & \mathbf{N}_{\alpha}^{2,0}=\left(i k \cdot \alpha_{1}\right)^{-1}\left(\partial_{T} \mathbf{N}_{\alpha}^{1,0}+\partial_{t} \mathbf{N}_{\alpha}^{0}-i\left[E^{0} \cdot \Gamma, \mathbf{C}^{1,0}\right]_{\mathrm{d}, \alpha}-W \sharp \mathbf{N}_{\alpha}^{0}\right) .
\end{array}
$$

Similarly, $\kappa>0$ leads to a polarization relation (using (37)), namely

$$
\forall \kappa>0, \quad \mathbf{N}^{2, \kappa}=\left(\gamma \kappa+k \cdot \partial_{\theta_{1}}\right)^{-1}\left(\partial_{T} \mathbf{N}^{1, \kappa}-i\left[E^{0} \cdot \Gamma, \mathbf{C}^{1}\right]_{\mathrm{d}}^{\kappa}-i\left[E^{1} \cdot \Gamma, \mathbf{C}^{0}\right]_{\mathrm{d}}^{\kappa}\right),
$$

which vanishes as soon as $\kappa>2$.

\subsection{Profile equations, intermediate scale analysis}

The next step in the analysis consists in obtaining a closed system determining the first profiles $\mathbf{u}^{0}, \mathbf{N}^{0}$ and $\mathbf{C}^{0}$. To achieve this, and in order to ensure consistancy of the Ansatz, we need impose that the corrector terms $\mathbf{u}^{1}, \mathbf{N}^{1}$ and $\mathbf{C}^{1}$ are small compared with the first profiles $\mathbf{u}^{0}, \mathbf{N}^{0}$ and $\mathbf{C}^{0}$. Concerning the field and the populations, this means we need impose $T$-sublinearity of the correctors 
$\mathbf{u}^{1,0}$ and $\mathbf{N}^{1,0}$ (as in [23], 26], 30]) while solving equations (50), (51), (58), and we shall prescribe the following requirement

$$
\forall \sigma \in \mathbb{N}^{3+2 d}, \quad \frac{1}{T} \sup _{t \in\left[0, t_{\star}\right]}\left\|\partial_{x, y, z, \theta}^{\sigma}\left(\mathbf{u}^{1,0}, \mathbf{N}^{1,0}\right)\right\|_{L^{2}} \underset{T \rightarrow+\infty}{\longrightarrow} 0 .
$$

Concerning coherences, i.e. while solving (57), we cannot impose the same constraint on $\mathbf{C}^{1,1}$. However, and as explained before, this corrector produces in the approximate solution a term $\sqrt{\varepsilon} \mathbf{C}^{1,1}(t, x, y, z, T, \theta)_{\left.\right|_{T=t / \sqrt{\varepsilon}}} e^{-\gamma t / \varepsilon}$, so that the possible growth in $T$ of $\mathbf{C}^{1,1}$ is eventually compensated by the factor $\exp (-\gamma t / \varepsilon)$. This is proved in section 4.3 .2 .

\subsubsection{Analysis of fields and populations: average operators}

The key observation for solving equations (50), (51), and (58) on $\mathbf{u}_{0}^{1,0}, \Pi \mathbf{u}_{\text {osc }}^{1,0}$, and $\mathbf{N}_{0}^{1,0}$, while keeping $T$-sublinear solutions $\mathbf{u}_{0}^{1,0}, \Pi \mathbf{u}_{\text {osc }}^{1,0}$, and $\mathbf{N}_{0}^{1,0}$, is that the source terms in these equations have a precise stucture in terms of propagation at the intermediate scale $T$.

Let us make our point precise. Our analysis is in three steps.

Firstly, equations (50), (51), and (58) are of the form

$$
\partial_{T} \Pi \mathbf{u}_{\text {osc }}^{1,0}=\cdots, \quad \partial_{T} \mathbf{N}_{0}^{1,0}=\cdots, \quad \partial_{T} \mathbf{u}_{0}^{1,0}+M_{2}\left(0, \partial_{y}, \partial_{z}\right) \mathbf{u}_{0}^{1,0}=\cdots,
$$

where the right-hand sides only depend on the lower order terms $\mathbf{u}^{0}$ and $\mathbf{N}^{0}$. Besides, for $(\eta, \zeta) \in$ $\mathbb{R}^{2} \backslash\{0\}$, the symmetric matrix $M_{2}(0, \eta, \zeta)$ has the spectral decomposition

$$
M_{2}(0, \eta, \zeta)=\sum_{k=0,+,-} \lambda_{k}(\eta, \zeta) p_{k}(\eta, \zeta)
$$

where the eigenvalues $\lambda_{k}$ are smooth on $\mathbb{R}^{2} \backslash\{0\}$ and homogeneous of degree 1 , with values

$$
\lambda_{0}(\eta, \zeta)=0, \quad \lambda_{+}(\eta, \zeta)=\sqrt{\eta^{2}+\zeta^{2}}, \quad \lambda_{-}(\eta, \zeta)=-\sqrt{\eta^{2}+\zeta^{2}}
$$

and the projectors $p_{k}$ are smooth on $\mathbb{R}^{2} \backslash\{0\}$ and homogeneous of degree 0 , with the following values

$$
\begin{aligned}
& p_{0} \text { is the orthogonal projector onto } \operatorname{Span}\left(\left(\begin{array}{c}
0 \\
\mathcal{Z}
\end{array}\right),\left(\begin{array}{c}
\mathcal{Z} \\
0
\end{array}\right)\right) \\
& p_{ \pm} \text {is the orthogonal projector onto } \operatorname{Span}\left(\left(\begin{array}{c} 
\pm|\mathcal{Z}| e_{x} \\
\mathcal{Z}^{\perp}
\end{array}\right),\left(\begin{array}{c}
\mathcal{Z}^{\perp} \\
\pm|\mathcal{Z}| e_{x}
\end{array}\right)\right) \\
& \text { where } \quad \mathcal{Z}=\left(\begin{array}{l}
0 \\
\eta \\
\zeta
\end{array}\right), \quad \mathcal{Z}^{\perp}=\left(\begin{array}{c}
0 \\
-\zeta \\
\eta
\end{array}\right), \quad e_{x}=\left(\begin{array}{c}
1 \\
0 \\
0
\end{array}\right) .
\end{aligned}
$$

Using Fourier transform, this allows to state the

Lemma 4.9. Take $u^{\text {in }} \in H^{\infty}\left(\mathbb{R}^{2}, \mathbb{C}^{6}\right)$.

Then, the unique solution $u \in \mathcal{C}\left(\mathbb{R}, H^{\infty}\right)$ to the Cauchy problem

$$
\partial_{T} u+M_{2}\left(0, \partial_{y}, \partial_{z}\right) u=0, \quad u_{\mid T=0}=u^{\text {in }},
$$


is given by

$$
u=\sum_{k=0,+,-} u_{k}
$$

where each $u_{k}=p_{k}\left(\mathrm{D}_{y}, \mathrm{D}_{z}\right) u$ is characterized by

$$
\left(\partial_{T}+i \lambda_{k}\left(\mathrm{D}_{y}, \mathrm{D}_{z}\right)\right) u_{k}=0, \quad u_{\left.k\right|_{T=0}}=p_{k}\left(\mathrm{D}_{y}, \mathrm{D}_{z}\right) u^{\mathrm{in}} .
$$

In passing, Lemma 4.9 implies equation (38) induces for $\mathbf{u}_{0}^{0}$ the splitting

$$
\mathbf{u}_{0}^{0}=\mathbf{u}_{0,0}^{0}+\mathbf{u}_{0,+}^{0}+\mathbf{u}_{0,-}^{0},
$$

with

$$
\mathbf{u}_{0, k}^{0}=p_{k}\left(\mathrm{D}_{y}, \mathrm{D}_{z}\right) \mathbf{u}_{0}^{0}, \quad\left(\partial_{T}+i \lambda_{k}\left(\mathrm{D}_{y}, \mathrm{D}_{z}\right)\right) \mathbf{u}_{0, k}^{0}=0 \quad(k=0,+,-) .
$$

Lemma 4.9 also implies that equations (50), (51), and (58) have the form

$$
\begin{gathered}
\left(\partial_{T}+i \lambda_{k}\left(D_{y}, D_{z}\right)\right) \mathbf{u}_{0, k}^{1,0}=\cdots \quad(k=0,+,-), \\
\left(\partial_{T}+i \lambda_{0}\left(D_{y}, D_{z}\right)\right) \Pi \mathbf{u}_{\mathrm{osc}}^{1,0}=\cdots, \quad\left(\partial_{T}+i \lambda_{0}\left(D_{y}, D_{z}\right)\right) \mathbf{N}_{0}^{1,0}=\cdots,
\end{gathered}
$$

where the various right-hand-sides only depend on $\mathbf{u}^{0}$ and $\mathbf{N}^{0}$.

With this observation in mind, the next step consists in analyzing the above equations using the average operators introduced in [30]. They allow to describe nonlinear interactions between the various modes $0,+,-$ in the equations at hand.

For each $k=0,+,-$, and $u \in \mathcal{C}\left(\left[0, t_{\star}\right]_{t} \times \mathbb{R}_{T}, H^{\infty}\left(\mathbb{R}_{x, y, z}^{3} \times \mathbb{T}_{\theta}^{2 d}\right)\right)$, we define (omitting the dependence upon $t, x$ and $\theta$ )

$$
G_{k}^{S} u(T, y, z):=\frac{1}{S} \int_{0}^{S} \mathcal{F}^{-1}\left(e^{i s \lambda_{k}(\eta, \zeta)} \hat{u}(T+s, \eta, \zeta)\right) \mathrm{d} s
$$

with $\mathcal{F}$ the Fourier transform in variables $y, z$. We also define the limit (if it exists),

$$
G_{k} u(T, y, z):=\lim _{S \rightarrow+\infty} G_{k}^{S} u(T, y, z) .
$$

The average operator $G_{k}$ performs the average along the bicharacteristic curves of the operator $\left(\partial_{T}+i \lambda_{k}\left(\mathrm{D}_{y}, \mathrm{D}_{z}\right)\right)$. Naturally, $G_{0}$ coincides with the usual average with respect to $T$, due to $\lambda_{0}=0$.

The following properties of the average operators $G_{k}$ are useful.

Proposition 4.10 (borrowed from [30]). Let $k \in\{0,+,-\}$.

(i) If $u \in \mathcal{C}\left(\left[0, t_{\star}\right]_{t} \times \mathbb{R}_{T}, H^{\infty}\left(\mathbb{R}_{x, y, z}^{3} \times \mathbb{T}_{\theta}^{2 d}\right)\right)$ satisfies] $\left(\partial_{T}+i \lambda_{k}\left(\mathrm{D}_{y}, \mathrm{D}_{z}\right)\right) u=0$, then $G_{k} u=u$.

(ii) If $f \in \mathcal{C}\left(\left[0, t_{\star}\right]_{t} \times \mathbb{R}_{T}, H^{\infty}\left(\mathbb{R}_{x, y, z}^{3} \times \mathbb{T}_{\theta}^{2 d}\right)\right)$ satisfies $G_{k} f=0$, then any solution $u$ to $\left(\partial_{T}+\right.$ $\left.i \lambda_{k}\left(\mathrm{D}_{y}, \mathrm{D}_{z}\right)\right) u=f$ is T-sublinear.

(iii) If $u \in \mathcal{C}^{1}\left(\left[0, t_{\star}\right]_{t} \times \mathbb{R}_{T}, H^{\infty}\left(\mathbb{R}_{x, y, z}^{3} \times \mathbb{T}_{\theta}^{2 d}\right)\right)$ is T-sublinear, then we have $G_{k}\left(\partial_{T}+i \lambda_{k}\left(\mathrm{D}_{y}, \mathrm{D}_{z}\right)\right) u=0$.

(iv) Let a collection $\left(u_{\ell}\right)_{0 \leq \ell \leq L} \subset \mathcal{C}\left(\left[0, t_{\star}\right]_{t} \times \mathbb{R}_{T}, H^{\infty}\left(\mathbb{R}_{x, y, z}^{3} \times \mathbb{T}_{\theta}^{2 d}\right)\right)$ satisfy $\left(\partial_{T}+i \lambda_{k_{\ell}}\left(\mathrm{D}_{y}, \mathrm{D}_{z}\right)\right) u_{\ell}=0$ with $k_{\ell} \in\{0,+,-\}$ for all $\ell$, and set $u:=u_{0} \cdots u_{L}$.

Then, if $k_{\ell}=k$ for all $\ell$, we have $G_{k} u=u$, else, if $k_{\ell} \neq k$ for some index $\ell$, we have $G_{k} u=0$.

\footnotetext{
${ }^{2}$ Here, solutions to equations of the form $\left(\partial_{T}+i \lambda_{k}\left(\mathrm{D}_{y}, \mathrm{D}_{z}\right)\right) u=f$ are meant in the mild sense, see remark 4.13 .
} 
Our last step consists in applying all above considerations. Indeed, Proposition 4.10 (ii)-(iii) asserts that equation $\left(\partial_{T}+i \lambda_{k}\left(\mathrm{D}_{y}, \mathrm{D}_{z}\right)\right) u=f$ possesses a $T$-sublinear solution if and only if $G_{k} f=0$, while Proposition 4.10 (iv) allows to explicitely compute $G_{k} f$ when $f$ is a product of solutions to $\left(\partial_{T}+i \lambda_{k_{\ell}}\left(\mathrm{D}_{y}, \mathrm{D}_{z}\right)\right) u_{\ell}=0$.

Concerning equation (50), with the wave structure given by (38), we conclude that equation (50) possesses a $T$-sublinear solution $\mathbf{u}_{0}^{1,0}$ if and only if

$$
p_{k}\left(\mathrm{D}_{y}, \mathrm{D}_{z}\right) M_{1}\left(\partial_{t}, \partial_{x}\right) p_{k}\left(\mathrm{D}_{y}, \mathrm{D}_{z}\right) \mathbf{u}_{0, k}^{0}=0 \quad(k=0,+,-) .
$$

These three systems in fact reduce to the trivial condition

$$
\partial_{t} \mathbf{u}_{0,0}^{0}=0, \quad \partial_{t} \mathbf{u}_{0,+}^{0}=0, \quad \partial_{t} \mathbf{u}_{0,-}^{0}=0,
$$

thanks to the following Lemma whose proof is a straightforward computation.

Lemma 4.11. Take $k \in\{0,+,-\}$ and $(\eta, \zeta) \in \mathbb{R}^{2} \backslash\{0\}$. Then, we have

$$
p_{k}(\eta, \zeta) A_{x} p_{k}(\eta, \zeta)=0 .
$$

Concerning equation (51), the right-hand-side of this equation only involves (products of) solutions to $\left(\partial_{T}+i \lambda_{k_{\ell}}\left(\mathrm{D}_{y}, \mathrm{D}_{z}\right)\right) u_{\ell}=0$, amongst which only the parts $\mathbf{u}_{\mathrm{osc}}^{0}, \mathbf{u}_{0,0}^{0}$ and $\mathbf{N}^{0}$ are associated with the characteristic speed $\lambda_{0}=0$, thanks to relations (39) and (47), and thanks to the definition of $\mathbf{u}_{0,0}^{0}$ in (64). Therefore, equation (51) possesses a $T$-sublinear solution $\mathbf{u}_{\text {osc }}^{1,0}$ if and only if

$$
\begin{aligned}
& \left(\partial_{t}+\mathrm{v}\left(\mathrm{D}_{\theta}\right) \partial_{x}\right) \mathbf{u}_{\mathrm{osc}}^{0}-i \mathrm{a}\left(\mathrm{D}_{\theta}\right) \Delta_{y, z} \mathbf{u}_{\mathrm{osc}}^{0}= \\
& \quad \Pi\left(0, i \operatorname{Tr}\left(\Gamma\left(i \Omega_{\gamma}\left(i \Omega_{\gamma}-k \cdot \partial_{\theta_{1}}\right)^{-1}-1\right)\left[\left(E_{0,0}^{0}+E_{\mathrm{osc}}^{0}\right) \cdot \Gamma, \mathbf{N}^{0}\right]_{\mathrm{osc}}\right)\right) .
\end{aligned}
$$

This equation may be transformed further. Indeed, when $\alpha \in \mathcal{C}_{0}$, we know from Lemma 4.5 and Lemma 4.6 that $\mathrm{v}(\alpha)=0$ and $\mathrm{a}(\alpha)=0$. Besides, we already know that the populations $\mathbf{N}^{0}$ only carry temporal oscillations, according to (37). As a consequence, we recover by a direct computation that equation (66) implies

$$
\left.\forall \alpha \in \mathcal{C}_{0}, \quad \partial_{t} \mathbf{u}_{\alpha}^{0}=0 . \quad \text { (i.e. } \quad \partial_{t} \mathbf{u}_{\text {space }}^{0}=0\right) .
$$

(This comes from the fact that the factor $\left.i \Omega_{\gamma}\left(i \Omega_{\gamma}-k \cdot \partial_{\theta_{1}}\right)^{-1}-1\right)$ vanishes when acting on a frequency $\alpha \in \mathcal{C}_{0}$ ). When $\alpha \in \mathcal{C}_{ \pm}$at variance, equation (66) provides, using Lemma 4.5 and Lemma 4.6, the relation

$$
\begin{aligned}
\forall \alpha \in \mathcal{C}_{ \pm}, \quad\left(\partial_{t} \pm \partial_{x}\right) \mathbf{u}_{\alpha}^{0} \mp & i \frac{1}{2 k \cdot \alpha_{0}} \Delta_{y, z} \mathbf{u}_{\alpha}^{0}= \\
& \Pi\left(0, i \operatorname{Tr}\left(\Gamma\left(\Omega_{\gamma}\left(\Omega_{\gamma}-k \cdot \alpha_{1}\right)^{-1}-1\right)\left[\left(E_{0,0}^{0}+E_{\mathrm{osc}}^{0}\right) \cdot \Gamma, \mathbf{N}^{0}\right]_{\alpha}\right)\right) .
\end{aligned}
$$

Lastly, concerning equation (58), the right-hand-side involves in the similar fashion only products of the profiles $\mathbf{N}_{\alpha}^{0}, E^{0}$, and $\mathbf{C}^{1,0}$, which, considering the relation (43), reduces to products of the profiles $\mathbf{N}^{0}$ and $E^{0}$. Amongst these profiles, only the parts $\mathbf{N}^{0}, E_{\mathrm{osc}}^{0}$, and $E_{0,0}^{0}$ are associated with the characteristic speed $\lambda_{0}=0$. Therefore, equation (58) possesses a $T$-sublinear solution $\mathbf{N}_{\alpha}^{1,0}$ if and only if (here we plug relation (43))

$$
\begin{aligned}
& \forall \alpha \in \mathcal{C}_{0} \cup\{0\}, \\
& \quad \partial_{t} \mathbf{N}_{\alpha}^{0}=W \sharp \mathbf{N}_{\alpha}^{0}-\left[\left(E_{0,0}^{0}+E_{\mathrm{osc}}^{0}\right) \cdot \Gamma,\left(i \Omega_{\gamma}-k \cdot \partial_{\theta_{1}}\right)^{-1}\left[\left(E_{0,0}^{0}+E_{\mathrm{osc}}^{0}\right) \cdot \Gamma, \mathbf{N}^{0}\right]_{\mathrm{od}}\right]_{\mathrm{d}, \alpha} .
\end{aligned}
$$


Eventually, we have now obtained the set of equations (65), (67), (68), and (69) as a set of necessary and sufficient conditions to be able to find $T$-sublinear solutions to (50), (51), and (58), respectively. This completes our effort in finding a system that completely determines the dominant profiles $\mathbf{u}^{0}$ and $\mathbf{N}^{0}$. Note in passing that equation (68) in fact reduces to

$$
\begin{aligned}
& \forall \alpha \in \mathcal{C}_{ \pm}, \quad\left(\partial_{t} \pm \partial_{x}\right) \mathbf{u}_{\alpha}^{0} \mp i \frac{1}{2 k \cdot \alpha_{0}} \Delta_{y, z} \mathbf{u}_{\alpha}^{0}= \\
& \quad \Pi\left(0, i \operatorname{Tr}\left(\Gamma\left(\Omega_{\gamma}\left(\Omega_{\gamma}-k \cdot \alpha_{1}\right)^{-1}-1\right)\left[E_{\text {time }}^{0} \cdot \Gamma, \mathbf{N}^{0}\right]_{\alpha}\right)\right),
\end{aligned}
$$

due to the fact that $\mathbf{N}_{\alpha}^{0}=0$ whenever $\alpha \notin \mathcal{C}_{0} \cup\{0\}$.

In conclusion, we have recovered equations (24), (26), (27), (28), (29), (30), (31) that had been announced in the introductory part of this paper.

\subsubsection{Analysis of coherences: exponential growth}

The above analysis is not possible in the case of (57) : equation (46), ruling the evolution of $\mathbf{C}^{0,1}$ as a function of $T$, is not a constant coefficient system. Instead, we consider it as a non-autonomous system of linear ODE's, parametrized by $t, x, y, z, \theta$, with smooth and bounded coefficients. This point of view provides the simple

Lemma 4.12. Let $\left(\mathbf{u}^{0}, \mathbf{N}^{0}\right) \in \mathcal{C}\left(\left[0, t_{\star}\right]_{t} \times \mathbb{R}_{T}, H^{\infty}\left(\mathbb{R}_{x, y, z}^{3} \times \mathbb{T}_{\theta}^{2 d}\right)\right)$ be a mild solution to (38), (39), (47), (65), (66), (69). Then,

(i) $\left(\mathbf{u}^{0}, \mathbf{N}^{0}\right) \in \mathcal{C}_{\mathrm{b}}^{\infty}\left(\left[0, t_{\star}\right]_{t} \times \mathbb{R}_{T}, H^{\infty}\left(\mathbb{R}_{x, y, z}^{3} \times \mathbb{T}_{\theta}^{2 d}\right)\right)$ is smooth and bounded uniformly with respect to all variables, as well as all its derivatives.

(ii) associated with these values of $\left(\mathbf{u}^{0}, \mathbf{N}^{0}\right)$, take any solution $\mathbf{C}^{0,1}$ to (46) with an initial data belonging to $\mathcal{C}^{\infty}\left(\left[0, t_{\star}\right]_{t}, H^{\infty}\left(\mathbb{R}_{x, y, z}^{3} \times \mathbb{T}_{\theta}^{2 d}\right)\right)$. Then, for any multi-index $\mu \in \mathbb{N}^{5+2 d}$, there are constants $K_{1}, K_{2}>0$ such that, uniformly on $\left[0, t_{\star}\right]_{t} \times \mathbb{R}_{T} \times \mathbb{R}_{x, y, z}^{3} \times \mathbb{T}_{\theta}^{2 d}$, we have

$$
\left|\partial_{t, T, x, y, z, \theta}^{\mu} \mathbf{C}^{0,1}(T)\right| \leq K_{1} e^{K_{2} T} .
$$

Remark 4.13. All equations that are referred to here are either of the form $\partial_{t} v=f\left(v, D v, D^{2} v\right)$, or of the form $\partial_{T} v=f\left(v, D v, D^{2} v\right)$, where $v$ is assumed to have $H^{\infty}$ smoothness in $(x, y, z, \theta)$, where the symbol $D$ means differentiation with respect to $(x, y, z, \theta)$, and $f$ is a possibly non-linear function that depends on the equation. The notion of mild solution is then the standard one : we mean a solution to the integral equation $v(t)=\left.v\right|_{t=0}+\int_{0}^{t} f\left(v, D v, D^{2} v\right)(s) \mathrm{d} s$ or to $v(T)=\left.v\right|_{T=0}+$ $\int_{0}^{T} f\left(v, D v, D^{2} v\right)(S) \mathrm{d} S$.

Proof. Boundedness and smoothness of $\left(\mathbf{u}^{0}, \mathbf{N}^{0}\right)$ with respect to $(x, y, z, \theta)$ is obvious. Concerning the variables $t$ and $T$, we exploit the fact that $t$ belongs to a compact set. We also exploit the structure of the relevant equations. More precisely, $\mathbf{u}_{0}^{0}$ satisfies $\left(\partial_{T}+M_{2}\left(0, \partial_{y}, \partial_{z}\right)\right) \mathbf{u}_{0}^{0}=0$ and $\partial_{t} \mathbf{u}_{0}^{0}=0$. This, together with Lemma 4.9, provides boundedness and smoothness of $\mathbf{u}_{0}^{0}$ with respect to $t$ and $T$. Next, $\mathbf{N}^{0}$ satisfies $\partial_{T} \mathbf{N}^{0}=0$ together with equation (69), an equation of the form $\partial_{t} \mathbf{N}^{0}=f\left(\mathbf{u}^{0}, \mathbf{N}^{0}\right)$ where $f$ is smooth. This provides boundedness and smoothness of $\mathbf{N}^{0}$ with respect to $t$ and $T$. We have used here the Diophantine Hypothesis 3.3 to conclude that $f$ is smooth. Lastly, $\mathbf{u}_{\text {osc }}^{0}$ satisfies $\partial_{T} \mathbf{u}_{\text {osc }}^{0}=0$ together with equation (66), an equation of the form $\partial_{t} \mathbf{u}_{\mathrm{osc}}^{0}=f\left(\mathbf{u}_{\mathrm{osc}}^{0}, D_{\theta} \mathbf{u}_{\mathrm{osc}}^{0}, D_{\theta}^{2} \mathbf{u}_{\mathrm{osc}}^{0}, \mathbf{u}_{0}^{0}, \mathbf{N}^{0}\right)$, where $f$ is smooth. This provides boundedness and smoothness of $\mathbf{u}_{\text {osc }}^{0}$ with respect to $t$ and $T$.

Point (ii) now comes from the Gronwall lemma, together with the fact that equation (46) has the form $\partial_{T} \mathbf{C}^{0,1}=f\left(E^{0}, \mathbf{C}^{0,1}\right)$ where $f$ is bilinear. 
Remark 4.14. Under the assumptions of the above Lemma, pushing the analysis further, we may consider a solution $\mathbf{C}^{1,1}$ to (57), and look for the available estimates on $\mathbf{C}^{1,1}$. This function is associated with a solution $\mathbf{N}^{1,1}$ to (48). In view of the above Lemma, the latter obviously satisfies an exponential bound of the form $\left|\partial_{t, T, x, y, z, \theta}^{\mu} \mathbf{N}^{1,1}(T)\right| \leq K_{1} e^{K_{2} T}$, where $K_{1}$ and $K_{2}$ depend on $\sigma$ but not on $T$. Hence $\mathbf{C}^{1,1}$ satisfies similarly $\left|\partial_{t, T, x, y, z, \theta}^{\mu} \mathbf{C}^{1,1}(T)\right| \leq K_{1} e^{K_{2} T}$. As a consequence, we recover

$$
\left|\sqrt{\varepsilon} \mathbf{C}^{1,1}(t, x, y, z, t / \sqrt{\varepsilon}, \theta) e^{-\gamma t / \varepsilon}\right| \leq K_{1} \sqrt{\varepsilon} e^{\left(K_{2}-\gamma / \sqrt{\varepsilon}\right) t / \sqrt{\varepsilon}}=\mathcal{O}(\sqrt{\varepsilon}) .
$$

\subsection{Solving the profile equations}

\subsubsection{Computing the dominant profile $\mathrm{U}^{0}$.}

The first profile $\mathbf{U}^{0}=\left(\mathbf{u}^{0}, \mathbf{C}^{0}, \mathbf{N}^{0}\right)$ is constrained by the polarization conditions (35), (36), (37). Besides, its components are propagated in various ways. The average $\mathbf{u}_{0}^{0}$ does not depend on the slow time $t$ (equation (65)), and it satisfies the linear hyperbolic equation $\left(\partial_{T}+M_{2}\left(0, \partial_{y}, \partial_{z}\right)\right) \mathbf{u}_{0}^{0}=0$ with respect to the intermediate time $T$ (equation (64)). The oscillating part $\mathbf{u}_{\mathrm{osc}}^{0}$, and the populations $\mathbf{N}^{0}$, do not depend on $T$ (equations (39), (47)), and they satisfy nonlinear evolution equations with respect to time $t$ (equations (66), (69)). Coherences $\mathbf{C}^{0}$ are only constrained to satisfy the nonlinear ODE (46) in time $T$, and the slow time $t$ only acts as a parameter here.

We are now in position to state the

Theorem 4.15. Let $s>(3+2 d) / 2$. Take a function

$$
\underline{\mathbf{U}}^{0} \equiv \underline{\mathbf{U}}^{0}\left(x, y, z, \theta_{0}\right),
$$

which belongs to $H^{s}\left(\mathbb{R}_{x, y, z}^{3} \times \mathbb{T}_{\theta_{0}}^{d}\right)$. Assume that the component $\underline{\mathbf{C}}^{0}$ of $\underline{\mathbf{U}}^{0}$ satisfies the following polarization condition

$$
\forall(m, n) \in \mathbb{Z}^{2 d} \text { satisfying }(m, n, \alpha) \notin \mathcal{R}(k) \text { for all } \alpha \in \mathbb{Z}^{2 d}, \quad \text { we have } \quad \underline{\mathbf{C}}_{m, n}^{0}=0 .
$$

Then, there is $t_{\star}>0$ and a unique function

$$
\mathbf{U}^{0}=\left(\mathbf{u}^{0}, \mathbf{C}^{0,1} e^{-\sigma}, \mathbf{N}^{0}\right) \in \mathcal{C}\left(\left[0, t_{\star}\right]_{t} \times\left[0,+\infty\left[{ }_{T} \times\left[0,+\infty\left[{ }_{\sigma}, H^{s}\left(\mathbb{R}_{x, y, z}^{3} \times \mathbb{T}_{\left(\theta_{0}, \theta_{1}\right)}^{2 d}\right)\right),\right.\right.\right.\right.
$$

solution to the polarization conditions (35), (36), (37), and which satisfies equations (64), (65) for the average field $\mathbf{u}_{0}^{0}$, equations (39), (66) for the oscillatory field $\mathbf{u}_{\mathrm{osc}}^{0}$, equations (47), (69) for the populations $\mathbf{N}^{0}$, and equation (46) for the coherences $\mathbf{C}^{0,1}$. Uniqueness and existence is guaranteed provided we impose $\mathbf{U}^{0}$ satisfies besides the two initial constraints

$$
\mathbf{U}_{\left.\right|_{t=T=\sigma=0, \theta_{1}=0} ^{0}}^{0}=\underline{\mathbf{U}}^{0}, \quad \text { and }\left.\mathbf{C}^{0,1}\right|_{T=0} \text { is independent of } t \text {. }
$$

If in addition we take an integer $\ell \in \mathbb{N}$ and assume that $s>(3+2 d) / 2+\ell(a+2)$, where the positive real $a$ is the one entering the Diophantine Hypothesis 3.马, then we recover the higher regularity

$$
\mathbf{U}^{0} \in \mathcal{C}^{\ell}\left(\left[0, t_{\star}\right]_{t} \times\left[0,+\infty\left[{ }_{T} \times\left[0,+\infty\left[{ }_{\sigma}, H^{s-\ell(a+2)}\left(\mathbb{R}_{x, y, z}^{3} \times \mathbb{T}_{\left(\theta_{0}, \theta_{1}\right)}^{2 d}\right)\right)\right.\right.\right.\right.
$$

\footnotetext{
${ }^{3}$ The reader should be cautious about the fact that the initial datum only depends on $\theta_{0}$, and not on $\theta_{1}$. Recall that eventually $\theta_{0}$ will be replaced by $k x / \varepsilon$ while $\theta_{1}$ takes the value $-k t / \varepsilon$.

${ }^{4}$ Due to the fact that the coherences only need to satisfy an evolution equation in the intermediate time $T$, note that the slow time $t$ needs to be treated separately here, including in terms of initial data. Note also that the initial data is not decomposed into modes, contrary to the solution itself.
} 


\section{Proof.}

First step : identifying the initial values of the profiles.

The first and most important step consists in understanding the polarization conditions, and how they generate the relevant initial data for the different modes. The data $\underline{\mathbf{U}}^{0}\left(x, y, z, \theta_{0}\right)$ is the value of the solution $\mathbf{U}^{0, \kappa}$ at $t=T=\sigma=0$ and $\theta_{1}=0$. We prove that it defines the value of $\mathbf{U}^{0, \kappa}$ at $t=T=0$ for all values of $\theta_{1} \in \mathbb{T}^{d}$.

Firstly, concerning the fields, the polarization constraint (35) provides, for the (to be defined) solution $\mathbf{u}^{0}$, the relations $\mathbf{u}^{0}=\mathbf{u}^{0,0}$ and $\Pi \mathbf{u}^{0}=\mathbf{u}^{0}$. On the other hand, we may write down the Fourier transform of $\underline{\mathbf{u}}^{0}$ in the sole variable $\theta_{0}$, together with the Fourier transform of $\mathbf{u}^{0}$ in $\left(\theta_{0}, \theta_{1}\right)$, and obtain

$$
\underline{\mathbf{u}}^{0}\left(x, y, z, \theta_{0}\right)=\sum_{\beta \in \mathbb{Z}^{d}} \underline{\mathbf{u}}_{\beta}^{0}(x, y, z) e^{i \beta \cdot \theta_{0}}, \quad \mathbf{u}^{0}\left(t, T, \sigma, x, y, z, \theta_{0}, \theta_{1}\right)=\sum_{\alpha \in \mathcal{C} \cup\{0\}} \mathbf{u}_{\alpha}^{0}(t, T, x, y, z) e^{i \alpha \cdot\left(\theta_{0}, \theta_{1}\right)} .
$$

Taking $t=T=\sigma=0, \theta_{1}=0$ in the second equation, and equating Fourier coefficients, then provides

$$
\forall \beta \in \mathbb{Z}^{d} \backslash\{0\}, \quad \underline{\mathbf{u}}_{\beta}^{0}=\left.\left(\mathbf{u}_{(\beta, \beta)}^{0}+\mathbf{u}_{(\beta,-\beta)}^{0}+\mathbf{u}_{(\beta, 0)}^{0}\right)\right|_{t=T=0}, \quad \underline{\mathbf{u}}_{0}^{0}=\left.\mathbf{u}_{(0,0)}^{0}\right|_{t=T=0},
$$

where $\mathbf{u}_{(\beta, \beta)}^{0}=\pi^{+} \mathbf{u}_{(\beta, \beta)}^{0}$, and $\mathbf{u}_{(\beta,-\beta)}^{0}=\pi^{-} \mathbf{u}_{(\beta,-\beta)}^{0}$, and $\mathbf{u}_{(\beta, 0)}^{0}=\pi^{0} \mathbf{u}_{(\beta, 0)}^{0}$, thanks to the polarization conditions and using the value of $\Pi$ (see (34)). Hence, applying successively the three operators $\pi^{+}$, $\pi^{-}, \pi^{0}$ on both sides of the first equality, and using the obvious orthogonality relations $\pi^{+} \pi^{-}=0$ and so on, we recover the necessary relations

$$
\begin{aligned}
& \left.\mathbf{u}_{(0,0)}^{0}\right|_{t=T=0}=\underline{\mathbf{u}}_{0}^{0}, \quad \text { and, when } \beta \neq 0, \\
& \left.\quad \mathbf{u}_{(\beta, \beta)}^{0}\right|_{t=T=0}=\pi^{+} \underline{\mathbf{u}}_{\beta}^{0},\left.\quad \mathbf{u}_{(\beta,-\beta)}^{0}\right|_{t=T=0}=\pi^{-} \underline{\mathbf{u}}_{\beta}^{0},\left.\quad \mathbf{u}_{(\beta, 0)}^{0}\right|_{t=T=0}=\pi^{0} \underline{\mathbf{u}}_{\beta}^{0} .
\end{aligned}
$$

This terminates the analysis of the initial conditions for the fields.

Secondly, concerning the populations, the polarization constraint (37) provides the simpler relations $\mathbf{N}^{0}=\mathbf{N}^{0,0}$ and $\mathbf{N}_{\alpha}^{0}=0$ whenever $\alpha \notin \mathcal{C}_{0} \cup\{0\}$. Writing down the Fourier transforms of $\underline{\mathbf{N}}^{0}$ and $\mathbf{N}^{0}$ as in the previous paragraph then provides $\underline{\mathbf{N}}^{0}\left(x, y, z, \theta_{0}\right)=\sum_{\beta \in \mathbb{Z}^{d}} \underline{\mathbf{N}}_{\beta}^{0}(x, y, z) e^{i \beta \cdot \theta_{0}}$, and $\mathbf{N}^{0}\left(t, T, \sigma, x, y, z, \theta_{0}, \theta_{1}\right)=\sum_{\beta \in \mathbb{Z}^{d}} \mathbf{N}_{\beta}^{0}(t, T, x, y, z) e^{i \beta \cdot \theta_{0}}$. Specifying $t=T=\sigma=0, \theta_{1}=0$, and equating Fourier coefficients, gives the simple value

$$
\forall \beta \in \mathbb{Z}^{d},\left.\quad \mathbf{N}_{\beta}^{0}\right|_{t=T=0}=\underline{\mathbf{N}}_{\beta}^{0}
$$

This terminates the analysis of the initial conditions for the populations.

Lastly, concerning the coherences, the polarization conditions (36) asserts $\mathbf{C}_{m, n, \alpha}^{0,1}=0$ whenever $(m, n, \alpha) \notin \mathcal{R}(k)$. Writing down the natural expansions of $\mathbf{C}^{0}=\mathbf{C}^{0,1} e^{-\sigma}$ and $\underline{\mathbf{C}}^{0}$, we recover for each value of $m$ and $n$ the identities $\underline{\mathbf{C}}_{m, n}^{0}\left(x, y, z, \theta_{0}\right)=\sum_{\beta \in \mathbb{Z}^{d}} \underline{\mathbf{C}}_{m, n, \beta}^{0}(x, y, z) e^{i \beta \cdot \theta_{0}}$, together with $\mathbf{C}_{m, n}^{0}\left(t, T, \sigma, x, y, z, \theta_{0}, \theta_{1}\right)=\left(\sum_{\alpha \in \mathbb{Z}^{2 d}} \mathbf{C}_{m, n, \alpha}^{0,1}(t, T, x, y, z) e^{i \alpha \cdot\left(\theta_{0}, \theta_{1}\right)}\right) e^{-\sigma}$. Specifying $t=T=\sigma=0$, $\theta_{1}=0$, gives using the polarization conditions

$$
\forall \beta \in \mathbb{Z}^{d}, \quad \underline{\mathbf{C}}_{m, n, \beta}^{0}=\sum_{\alpha_{1} \in \mathbb{Z}^{d}} \mathbf{C}_{m, n,\left(\beta, \alpha_{1}\right)}^{0,1} .
$$

Now, two cases occur, depending on the value of $(m, n)$. 
- First case : for any $\alpha \in \mathbb{Z}^{2 d}$, we have $(m, n, \alpha) \notin \mathcal{R}(k)$.

In that case, equation (74) is automatically satisfied since the right-hand-side vanishes due to the polarization constraint on $\mathbf{C}^{0,1}$, while the left-hand-side is assumed to vanish thanks to the additional constraint (72) we have set on the initial function $\underline{\mathbf{C}}^{0}$.

- Second case : there exists an $\alpha \in \mathbb{Z}^{2 d}$, such that $(m, n, \alpha) \in \mathcal{R}(k)$.

In that case, the solution $\widetilde{\alpha}_{1} \in \mathbb{Z}^{d}$ to the equation $\omega(m, n)=k \cdot \widetilde{\alpha}_{1}$ is unique, thanks to the $\mathbb{Q}$-independence of the coordinates of the wave-vector $k$. Recall that this equation defines the set $\mathcal{R}(k)$. Therefore, given any $\beta \in \mathbb{Z}^{d}$, equation (74) reduces to

$$
\mathbf{C}_{m, n,\left(\beta, \widetilde{\alpha}_{1}\right)}^{0,1}=\underline{\mathbf{C}}_{m, n, \beta}^{0}, \quad \text { and } \forall \alpha_{1} \neq \widetilde{\alpha}_{1}, \quad \mathbf{C}_{m, n,\left(\beta, \alpha_{1}\right)}^{0,1}=0 .
$$

This terminates the analysis of the initial conditions for the coherences.

Second step : solving the evolution equations.

We now consider the evolution problem with respect to the times $t$ and $T$, with the above derived initial data.

The field average $\mathbf{u}_{0}^{0}$ decouples and may be determined first starting from the initial value $\mathbf{u}_{\left.0\right|_{t=T=0} ^{0}}$, by using $\left(\partial_{T}+M_{2}\left(0, \partial_{y}, \partial_{z}\right)\right) \mathbf{u}_{0}^{0}=0$ and $\partial_{t} \mathbf{u}_{0}^{0}=0$.

Next, we may solve the equations on $\left(\mathbf{u}_{\mathrm{osc}}^{0}, \mathbf{N}^{0}\right)$. The evolution with respect to $T$ is trivial since $\partial_{T}\left(\mathbf{u}_{\text {osc }}^{0}, \mathbf{N}^{0}\right)=0$. There remains to solve the coupled nonlinear system (66), (69), an evolution equation in $t$. To do so, we use a standard iterative scheme (see for example [1]), and introduce the iteration

$$
\begin{aligned}
& \left(\partial_{t}+\mathrm{v}\left(\mathrm{D}_{\theta}\right) \partial_{x}\right) \mathbf{u}_{\mathrm{osc}}^{0,(n+1)}-i \mathrm{a}\left(\mathrm{D}_{\theta}\right) \Delta_{y, z} \mathbf{u}_{\mathrm{osc}}^{0,(n+1)}= \\
& \Pi\left(0, i \operatorname{Tr}\left(\Gamma\left(\Omega_{\gamma}\left(\Omega_{\gamma}-k \cdot \partial_{\theta_{0}}\right)^{-1}-1\right)\left[E_{\mathrm{osc}}^{0,(n)} \cdot \Gamma, \mathbf{N}^{0,(n)}\right]_{\mathrm{osc}}\right)\right), \\
& \partial_{t} \mathbf{N}^{0,(n+1)}=W \sharp \mathbf{N}^{0,(n+1)}-\left[\left(E_{\mathrm{osc}}^{0,(n)}+E_{0,0}^{0}\right) \cdot \Gamma,\left(i \Omega_{\gamma}-k \cdot \partial_{\theta_{0}}\right)^{-1}\left[\left(E_{\mathrm{osc}}^{0,(n)}+E_{0,0}^{0}\right) \cdot \Gamma, \mathbf{N}^{0,(n)}\right]_{\mathrm{od}}\right]_{\mathrm{d}, \mathrm{space}} \\
& -\left[\left(E_{\mathrm{osc}}^{0,(n)}+E_{0,0}^{0}\right) \cdot \Gamma,\left(i \Omega_{\gamma}-k \cdot \partial_{\theta_{0}}\right)^{-1}\left[\left(E_{\mathrm{osc}}^{0,(n)}+E_{0,0}^{0}\right) \cdot \Gamma, \mathbf{N}^{0,(n)}\right]_{\mathrm{od}}\right]_{\mathrm{d}, 0},
\end{aligned}
$$

with initial data $\left(\left(\mathbf{u}_{\mathrm{osc}}^{0}\right)_{\mid t=T=0},\left.\mathbf{N}^{0}\right|_{t=T=0}\right)$, and an initial "guess" set to, say, $\left(\mathbf{u}_{\mathrm{osc}}^{0,(0)}, \mathbf{N}^{0,(0)}\right)=$ const $=$ $\left(\left(\mathbf{u}_{\mathrm{osc}}^{0}\right)_{\mid t=T=0}, \mathbf{N}^{0}{ }_{t=T=0}\right)$ for any $t$ (and $T$ ). For this linearized scheme, usual energy estimates are available in any Sobolev space $H^{s}$, to which the skew-symmetric operators $\mathrm{v}\left(\mathrm{D}_{\theta}\right) \partial_{x}$ and $i \mathrm{a}\left(\mathrm{D}_{\theta}\right) \Delta_{y, z}$ do not contribute. They are

$$
\left\|U^{0,(m+1)}(t)-U^{0,(n+1)}(t)\right\|_{H^{s}} \leq \int_{0}^{t} e^{C(t-s)}\left\|F\left(U^{0,(m)}\right)(s)-F\left(U^{0,(n)}\right)(s)\right\|_{H^{s}} \mathrm{~d} s,
$$

where $F\left(U^{0(n)}\right)$ stands for the right-hand-side in the above iteration. Now, standard nonlinear tools assert that the function $F$ acts in a locally Lipschitz fashion on $H^{s}=H^{s}\left(\mathbb{R}^{3} \times \mathbb{T}^{2 d}\right)$ provided $s>(3+2 d) / 2$. The existence and uniqueness of a solution $\left(\mathbf{u}_{\mathrm{osc}}^{0}, \mathbf{N}^{0}\right)$ to (66), (69) on some time interval $\left[0, t_{*}\right]$ then follows.

Concerning the coherences $\mathbf{C}^{0,1}$, the statement of our Theorem imposes to choose them independent of $t$ at time $T=0$. This constraint is only a (pratical) way to fix the value of $\mathbf{C}^{0,1}$ on the set $\{T=0\}$ (recall that $\mathbf{C}^{0,1}$ satisfies an evolution equation in time $T$ only). Equation (46) then asserts

$$
\partial_{T} \mathbf{C}^{0,1}=i\left[E^{0} \cdot \Gamma, \mathbf{C}^{0,1}\right]
$$


This equation is enough to uniquely determine $\mathbf{C}^{0,1}$ for any $t$ and $T$, starting from its known values on $\{T=0\}$.

The polarization conditions (35), (36), (37) commute with equations (64), (65) for the average field $\mathbf{u}_{0}^{0}$, with equations (39), (66) for the oscillatory field $\mathbf{u}_{\mathrm{osc}}^{0}$, with equations (47), (69) for the populations $\mathbf{N}^{0}$, and with equation (46) for the coherences $\mathbf{C}^{0,1}$. Hence by uniqueness of the solutions to these propagation equations, polarizations are preserved along the evolution.

There remains to study the higher regularity of the solutions we have exhibited. The equations on the mean field, the equations on the populations, and the equations on the coherences clearly do not induce any loss of smoothness, i.e. provided $\mathbf{u}_{\mathrm{osc}}^{0}$ is $H^{\infty}$, the derivatives $\partial_{t}\left(\mathbf{u}_{0}^{0}, \mathbf{C}^{0,1}, \mathbf{N}^{0}\right)$ and $\partial_{T}\left(\mathbf{u}_{0}^{0}, \mathbf{C}^{0,1}, \mathbf{N}^{0}\right)$ have the same $H^{s}$ smoothness as $\left(\mathbf{u}_{0}^{0}, \mathbf{C}^{0,1}, \mathbf{N}^{0}\right)$. The loss of smoothness comes from the Schrödinger-like equation (66) on $\mathbf{u}_{\mathrm{osc}}^{0}$. When differentiating this equation with respect to $t$ indeed, an additional factor a $(\alpha) \Delta_{y, z}= \pm \frac{1}{2 k \cdot \alpha_{0}} \Delta_{y, z}$ comes into play which causes, from the Diophantine Hypothesis 3.3, a loss of $s_{0}=a+2$ derivatives (two derivatives are lost due to the Laplacian, while $a$ derivatives are lost due to small divisors, seen in Fourier space). This argument explains why $\mathbf{U}^{0} \in \mathcal{C}^{1}\left(H^{s-s_{0}}\right)$ and, more generally, why $\mathbf{U}^{0} \in \mathcal{C}^{\ell}\left(H^{s-\ell s_{0}}\right)$.

\subsubsection{Computing the correctors $\mathrm{U}^{1}$ and $\mathrm{U}^{2}$.}

The first corrector $\mathbf{U}^{1}=\left(\mathbf{u}^{1}, \mathbf{C}^{1}, \mathbf{N}^{1}\right)$ is built up in the following way. On the one hand, we have

$$
\mathbf{u}^{1}=\mathbf{u}^{1,0}+\mathbf{u}^{1,1} e^{-\sigma}, \quad \mathbf{C}^{1}=\mathbf{C}^{1,0}+\mathbf{C}^{1,1} e^{-\sigma}, \quad \mathbf{N}^{1}=\left(\mathbf{N}_{0}^{1,0}+\mathbf{N}_{\text {space }}^{1,0}\right)+\mathbf{N}^{1,1} e^{-\sigma},
$$

thanks to (42), (44), (48). Besides, the contributions $(1-\Pi) \mathbf{u}_{\text {osc }}^{1,0}, \mathbf{u}^{1,1}, \mathbf{C}^{1,0}, \mathbf{C}_{m, n, \alpha}^{1,1} \mathbf{1}_{(m, n, \alpha) \notin \mathcal{R}(k)}$, $\mathbf{N}^{1,1}$, are prescribed as functions of the dominant profile $\mathbf{U}^{0}$ through the polarization conditions (40), (41), (43), (45), (48), respectively. They are thus known and smooth functions of $\mathbf{U}^{0}$. Let us write these relations symbolically as

$$
\left((1-\Pi) \mathbf{u}_{\mathrm{osc}}^{1,0}, \mathbf{u}^{1,1}, \mathbf{C}^{1,0}, \mathbf{C}_{m, n, \alpha}^{1,1} \mathbf{1}_{(m, n, \alpha) \notin \mathcal{R}(k)}, \mathbf{N}^{1,1}\right)=\phi\left(\mathbf{U}^{0}\right)
$$

The remaining terms

$$
\mathbf{u}_{0}^{1,0}, \quad \Pi \mathbf{u}_{\text {osc }}^{1,0}, \quad \mathbf{C}_{m, n, \alpha}^{1,1} \mathbf{1}_{(m, n, \alpha) \in \mathcal{R}(k)}, \quad \mathbf{N}_{0}^{1,0}+\mathbf{N}_{\text {space }}^{1,0}
$$

are then determined through linear evolution equations in $T$, namely through (50), (51), (57), (58), respectively. These equations read, after some simplifications,

$$
\begin{aligned}
& \left(\partial_{T}+M_{2}\left(0, \partial_{y}, \partial_{z}\right)\right) \mathbf{u}_{0}^{1,0}=-A_{x} \partial_{x} \mathbf{u}_{0}^{0,0}, \quad \partial_{T} \Pi \mathbf{u}_{\text {osc }}^{1,0}=0, \\
& \forall \alpha \in \mathcal{C}_{0} \cup\{0\}, \quad \partial_{T} \mathbf{N}_{\alpha}^{1,0}=-\left[E^{0} \cdot \Gamma,\left(i \Omega_{\gamma}-k \cdot \partial_{\theta_{0}}\right)^{-1}\left[E^{0} \cdot \Gamma, \mathbf{N}^{0}\right]_{\mathrm{od}}\right]_{\mathrm{d}, \alpha} \\
& +\left[\left(E_{\mathrm{osc}}^{0}+E_{0,0}^{0}\right) \cdot \Gamma,\left(i \Omega_{\gamma}-k \cdot \partial_{\theta_{0}}\right)^{-1}\left[\left(E_{\mathrm{osc}}^{0}+E_{0,0}^{0}\right) \cdot \Gamma, \mathbf{N}^{0}\right]_{\mathrm{od}}\right]_{\mathrm{d}, \alpha}, \\
& \forall(m, n, \alpha) \in \mathcal{R}(k), \quad \partial_{T} \mathbf{C}_{m, n, \alpha}^{1,1}=-\partial_{t} \mathbf{C}_{m, n, \alpha}^{0,1}+i\left[E^{0,0} \cdot \Gamma, \mathbf{C}^{1,1}+\mathbf{N}^{1,1}\right]_{m, n, \alpha} \\
& +i\left[E^{1,0} \cdot \Gamma, \mathbf{C}^{0,1}\right]_{m, n, \alpha}+i\left[E^{1,1} \cdot \Gamma, \mathbf{N}^{0,0}\right]_{m, n, \alpha} .
\end{aligned}
$$

Note that the right-hand-side of the above system only involves known functions of $\mathbf{U}^{0}$ at this stage. In order to solve this system, there remains to impose as we did for $\mathbf{C}^{0,1}$,

$$
\left.\left(\mathbf{u}_{0}^{1,0}, \Pi \mathbf{u}_{\mathrm{osc}}^{1,0}, \mathbf{N}_{\alpha}^{1,0} \mathbf{1}_{\alpha \in \mathcal{C}_{0} \cup\{0\}}, \mathbf{C}_{m, n, \alpha}^{1,1} \mathbf{1}_{(m, n, \alpha) \in \mathcal{R}(k)}\right)\right|_{T=0} \text { is independent of } t .
$$


This completely determines $\mathbf{U}^{1}$ as a function of $\mathbf{U}^{0}$.

The second corrector $\mathbf{U}^{2}=\left(\mathbf{u}^{2}, \mathbf{C}^{2}, \mathbf{N}^{2}\right)$ is built up in the following way. On the one hand, we have

$$
\mathbf{u}^{2}=\mathbf{u}^{2,0}+\mathbf{u}^{2,1} e^{-\sigma}, \quad \mathbf{C}^{2}=\mathbf{C}^{2,0}+\mathbf{C}^{2,1} e^{-\sigma}+\mathbf{C}^{2,2} e^{-2 \sigma}, \quad \mathbf{N}^{2}=\mathbf{N}^{2,0}+\mathbf{N}^{2,1} e^{-\sigma}+\mathbf{N}^{2,2} e^{-2 \sigma},
$$

thanks to (54), (55), (60). Besides, the contributions $(1-\Pi) \mathbf{u}_{\text {osc }}^{2,0}, \mathbf{u}^{2,1}, \mathbf{C}^{2,0}, \mathbf{C}_{m, n, \alpha}^{2,1} \mathbf{1}_{(m, n, \alpha) \notin \mathcal{R}(k)}, \mathbf{C}^{2,2}$, $\mathbf{N}_{\alpha}^{2,0} \mathbf{1}_{\alpha \notin \mathcal{C}_{0} \cup\{0\}}, \mathbf{N}^{2,1}, \mathbf{N}^{2,2}$, are prescribed as functions on the two first profiles $\mathbf{U}^{0}$ and $\mathbf{U}^{1}$ through the polarization conditions (52), (53), (55), (56), (55), (59), (60), (60), respectively. They are thus known, smooth functions of $\mathbf{U}^{0}$ and $\mathbf{U}^{1}$. Some parts of the corrector are free and may be chosen arbitrarily, namely $\mathbf{u}_{0}^{2,0}, \Pi \mathbf{u}_{\text {osc }}^{2,0}, \mathbf{C}_{m, n, \alpha}^{2,1} \mathbf{1}_{(m, n, \alpha) \in \mathcal{R}(k)}, \mathbf{N}_{\alpha}^{2,0} \mathbf{1}_{\alpha \in \mathcal{C}_{0} \cup\{0\}}$. We make the most simple choice to set these contributions to zero. Let us write all these relations symbolically as

$$
\mathbf{U}^{2}=\psi\left(\mathbf{U}^{0}, \mathbf{U}^{1}\right)
$$

All these considerations, in conjunction with Theorem 4.15, lead to the

Proposition 4.16. For all $\ell \in \mathbb{N}$, there exists $\tilde{s}(\ell, a)$ such that the following holds.

Take an $s>\tilde{s}+(3+2 d) / 2$. Take an initial data $\underline{\mathbf{U}}^{0}$ in $H^{s}\left(\mathbb{R}_{x, y, z}^{3} \times \mathbb{T}_{\theta_{0}}^{d}\right)$ which satisfies the constraint (72). Take the profile $\mathbf{U}^{0}$ associated with these data through Theorem 4.15. Take two initial data $\underline{\mathbf{U}}^{1}$ in $H^{s}\left(\mathbb{R}_{x, y, z}^{3} \times \mathbb{T}_{\theta_{0}}^{d}\right)$ and $\underline{\mathbf{U}}^{2}$ in $H^{s}\left(\mathbb{R}_{x, y, z}^{3} \times \mathbb{T}_{\theta_{0}}^{d}\right)$. Then, there are unique correctors

$$
\mathbf{U}^{1}=\left(\begin{array}{c}
\mathbf{u}^{1,0}+\mathbf{u}^{1,1} e^{-\sigma} \\
\mathbf{C}^{1,0}+\mathbf{C}^{1,1} e^{-\sigma} \\
\left(\mathbf{N}_{0}^{1,0}+\mathbf{N}_{\text {space }}^{1,0}\right)+\mathbf{N}^{1,1} e^{-\sigma}
\end{array}\right), \quad \mathbf{U}^{2}=\left(\begin{array}{c}
\mathbf{u}^{2,0}+\mathbf{u}^{2,1} e^{-\sigma} \\
\mathbf{C}^{2,0}+\mathbf{C}^{2,1} e^{-\sigma}+\mathbf{C}^{2,2} e^{-2 \sigma} \\
\mathbf{N}^{2,0}+\mathbf{N}^{2,1} e^{-\sigma}+\mathbf{N}^{2,2} e^{-2 \sigma}
\end{array}\right)
$$

in $\mathcal{C}^{\ell}\left(\left[0, t_{\star}\right]_{t} \times\left[0,+\infty\left[{ }_{T} \times\left[0,+\infty\left[{ }_{\sigma}, H^{s-\tilde{s}}\left(\mathbb{R}_{x, y, z}^{3} \times \mathbb{T}_{\left(\theta_{0}, \theta_{1}\right)}^{2 d}\right)\right)\right.\right.\right.\right.$, which satisfy the constraints (76), (78) for the first corrector, the constraints (80) for the second corrector, and the evolution equation (58) for the first corrector, together with the initial constraint

$$
\left.\mathbf{U}^{1}\right|_{T=t=\sigma=0, \theta_{1}=0}=\left.\underline{\mathbf{U}}^{1} \quad \mathbf{U}^{2}\right|_{T=t=\sigma=0, \theta_{1}=0}=\underline{\mathbf{U}}^{2} .
$$

Proof. The initial constraint $\left.\mathbf{U}^{1}\right|_{T=t=\sigma=0, \theta_{1}=0}=\underline{\mathbf{U}}^{1}$ reads

$$
\left.\left(\mathbf{u}^{1,0}+\mathbf{u}^{1,1}, \mathbf{C}^{1,0}+\mathbf{C}^{1,1},\left(\mathbf{N}_{0}^{1,0}+\mathbf{N}_{\text {space }}^{1,0}\right)+\mathbf{N}^{1,1}\right)\right|_{T=t=0, \theta_{1}=0}=\underline{\mathbf{U}}^{1} .
$$

This, together with (76) written at time $T=t=0$, prescribes the value of

$$
\left.\left((1-\Pi) \mathbf{u}_{\mathrm{osc}}^{1,0}, \mathbf{u}^{1,1}, \mathbf{C}^{1,0}, \mathbf{C}_{n, m, \alpha}^{1,1} \mathbf{1}_{(m, n, \alpha) \notin \mathcal{R}(k)}, \mathbf{N}^{1,1}\right)\right|_{T=t=0}=\phi\left(\underline{\mathbf{U}}^{0}\right) .
$$

Hence, taking the difference, we recover the value of

$$
\left.\left(\mathbf{u}_{0}^{1,0}+\Pi \mathbf{u}_{\mathrm{osc}}^{1,0}, \sum_{\alpha} \mathbf{C}_{n, m, \alpha}^{1,1} \mathbf{1}_{(m, n, \alpha) \in \mathcal{R}(k)} e^{i \alpha_{0} \cdot \theta_{0}}, \mathbf{N}_{0}^{1,0}+\mathbf{N}_{\text {space }}^{1,0}\right)\right|_{T=t=0, \theta_{1}=0},
$$


an explicit, linear function of $\underline{\mathbf{U}}^{0}$ and $\underline{\mathbf{U}}^{1}$. Fourier transforming $\underline{\mathbf{U}}^{0}$ and $\underline{\mathbf{U}}^{1}$ in the variable $\theta_{0}$, and Fourier transforming

$$
\left(\mathbf{u}_{0}^{1,0}+\Pi \mathbf{u}_{\mathrm{osc}}^{1,0}, \sum_{\alpha} \mathbf{C}_{n, m, \alpha}^{1,1} \mathbf{1}_{(m, n, \alpha) \in \mathcal{R}(k)} e^{\left.i\left(\alpha_{0} \cdot \theta_{0}+\alpha_{1} \cdot \theta_{1}\right)\right)}, \mathbf{N}_{0}^{1,0}+\mathbf{N}_{\text {space }}^{1,0}\right)
$$

in the variable $\left(\theta_{0}, \theta_{1}\right)$ then allows to deduce, as we did in the proof of Theorem 4.15, the value of $\left.\left(\mathbf{u}_{0}^{1,0}, \Pi \mathbf{u}_{\alpha}^{1,0}, \mathbf{C}_{m, n, \alpha^{\prime}}^{1,1}, \mathbf{N}_{\alpha^{\prime \prime}}^{1,0}\right)\right|_{T=t=0}$, whenever $\alpha \in \mathbb{Z}^{2 d} \backslash\{0\},\left(m, n, \alpha^{\prime}\right) \in \mathcal{R}(k)$, and $\alpha^{\prime \prime} \in \mathcal{C}_{0} \cup\{0\}$. The requirement (78) then gives the value of $\left(\mathbf{u}_{0}^{1,0}, \Pi \mathbf{u}_{\alpha}^{1,0}, \mathbf{C}_{m, n, \alpha^{\prime}}^{1,1}, \mathbf{N}_{\alpha^{\prime \prime}}^{1,0}\right)$ on the whole set $\{T=0\} \cup\{t=0\}$. Equation (58) in turn provides the value of $\left(\mathbf{u}_{0}^{1,0}, \Pi \mathbf{u}_{\alpha}^{1,0}, \mathbf{C}_{m, n, \alpha^{\prime}}^{1,1}, \mathbf{N}_{\alpha^{\prime \prime}}^{1,0}\right)$ for any value of $T$ and $t$. The remaining part of $\mathbf{U}^{1}$, namely the value of $\left((1-\Pi) \mathbf{u}_{\text {osc }}^{1,0}, \mathbf{u}^{1,1}, \mathbf{C}^{1,0}, \mathbf{C}_{n, m, \alpha}^{1,1} \mathbf{1}_{(m, n, \alpha) \notin \mathcal{R}(k)}, \mathbf{N}^{1,1}\right)$ for all values of $T$ and $t$, is next given by $\phi\left(\mathbf{U}^{0}\right)$. This terminates the computation of $\mathbf{U}^{1}$ for all values of $T$ and $t$. The relation $\mathbf{U}^{2}=\psi\left(\mathbf{U}^{0}, \mathbf{U}^{1}\right)$, see (80), prescribes $\mathbf{U}^{2}$ for al values of $T$ and $t$.

Let us come to regularity issues. Thanks to the constraint $(76)$, the $\mathcal{C}^{\ell}\left(H^{s-\tilde{s}(\ell, a)}\right)$ smoothness of $(1-\Pi) \mathbf{u}_{\text {osc }}^{1,0}, \mathbf{u}^{1,1}, \mathbf{C}^{1,0}, \mathbf{C}_{m, n, \alpha}^{1,1} \mathbf{1}_{(m, n, \alpha) \notin \mathcal{R}(k)}, \mathbf{N}^{1,1}$ is simply the consequence of the $\mathcal{C}^{\ell}\left(H^{s-\tilde{s}(\ell, a)}\right)$ smoothness of $\mathbf{U}^{0}$. For $\mathbf{u}_{0}^{1,0}, \Pi \mathbf{u}_{\text {osc }}^{1,0}, \mathbf{N}_{\alpha}^{1,0} \mathbf{1}_{\alpha \in \mathcal{C}_{0} \cup\{0\}}$ and $\mathbf{C}_{m, n, \alpha}^{1,1} \mathbf{1}_{(m, n, \alpha) \in \mathcal{R}(k)}$, the $\mathcal{C}^{0}\left(H^{s-\tilde{s}(0, a)}\right)$ regularity stems from the classical smoothness of solutions to linear hyperbolic systems with source terms lying in $\mathcal{C}^{0}\left(H^{s-\tilde{s}(0, a)}\right)$. Note that in the last equation in (58), the regularity of the source term $\partial_{t} \mathbf{C}^{0,1}$ is a simple consequence of the regularity of solutions to ODE's depending on a parameter (here $t$ ). Note also that the overall loss $\tilde{s}(0, a)$ comes from both the involved derivatives of $\mathbf{U}^{0}$ appearing in the source terms, and from small divisors $\left(\omega(m, n)-k \cdot \alpha_{1}\right)^{-1}$ and $M_{1}\left(-k \cdot \alpha_{1}, k \cdot \alpha_{0}\right)^{-1}$ acting on $\mathbf{U}^{0}$, that appear in the source term as well. Differentiating (77) and (76) with respect to $T$ and $t$, and applying the same argument, eventually provides the $\mathcal{C}^{\ell}\left(H^{s-\tilde{s}(\ell, a)}\right)$ smoothness of $\mathbf{U}^{1}$. Relation $\mathbf{U}^{2}=\psi\left(\mathbf{U}^{0}, \mathbf{U}^{1}\right)$ provides the $\mathcal{C}^{\ell}\left(H^{s-\tilde{s}(\ell, a)}\right)$ smoothness of $\mathbf{U}^{2}$.

Remark 4.17. One can prove that for any $\ell$ we have $\mathbf{U}^{1} \in \mathcal{C}^{\ell}\left(H^{s-\max (3 a+1, a+2)-\ell(a+2)}\right)$, and $\mathbf{U}^{2} \in$ $\mathcal{C}^{\ell}\left(H^{s-\max (4 a+2,3 a+3)-\ell(a+2)}\right)$.

\section{Convergence}

\subsection{The residual}

Writing down the full profile representation of the residual

$$
r^{\varepsilon}(t, x, y, z)=L\left(\partial_{t}, \partial_{x}, \frac{1}{\sqrt{\varepsilon}} \partial_{y}, \frac{1}{\sqrt{\varepsilon}} \partial_{z}\right) \mathbf{U}_{\mathrm{app}}^{\varepsilon}-F^{\varepsilon}\left(\mathbf{U}_{\mathrm{app}}^{\varepsilon}\right),
$$

we have, using Proposition 4.2,

$$
\begin{aligned}
& r^{\varepsilon}(t, x, y, z)=\left.\mathcal{R}^{\varepsilon}(t, x, y, z, T, \sigma, \theta)\right|_{T=t / \sqrt{\varepsilon}, \sigma=\gamma t / \varepsilon,\left(\theta_{0}, \theta_{1}\right)=(k x,-k t) / \varepsilon}, \\
& \text { with } \mathcal{R}^{\varepsilon}=\sum_{j=-2}^{3} \sum_{\kappa \geq 0} \sqrt{\varepsilon} r^{j, \kappa}(t, x, y, z, T, \theta) \exp (-\kappa \sigma) .
\end{aligned}
$$


We now find how $r^{1}, r^{2}$ and $r^{3}$ depend on the profiles $\mathbf{U}^{1}, \mathbf{U}^{2}$ and $\mathbf{U}^{3}$. Symbolically, introducing first order differential operators $L_{1}$ and $L_{2}$, a matrix $L_{0}$, and some bounded, symmetric, bilinear operators $B_{E}, B_{(\mathbf{C}, \mathbf{N})}$ (with values on the $E$ - and $(\mathbf{C}, \mathbf{N})$-components, respectively), we have

$$
\begin{aligned}
& r^{1}=L_{2}\left(\partial_{T}, \partial_{y}, \partial_{z}\right) \mathbf{U}^{2}+L_{1}\left(\partial_{t}, \partial_{x}\right) \mathbf{U}^{1}+2 B_{E}\left(\mathbf{U}^{0}, \mathbf{U}^{1}\right)+2 B_{(\mathbf{C}, \mathbf{N})}\left(\mathbf{U}^{0}, \mathbf{U}^{2}\right)+B_{(\mathbf{C}, \mathbf{N})}\left(\mathbf{U}^{1}, \mathbf{U}^{1}\right)+L_{0} \mathbf{U}^{1} \\
& r^{2}=L_{1}\left(\partial_{t}, \partial_{x}\right) \mathbf{U}^{2}+2 B_{E}\left(\mathbf{U}^{0}, \mathbf{U}^{2}\right)+B_{E}\left(\mathbf{U}^{1}, \mathbf{U}^{1}\right)+2 B_{(\mathbf{C}, \mathbf{N})}\left(\mathbf{U}^{1}, \mathbf{U}^{2}\right)+L_{0} \mathbf{U}^{2} \\
& r^{3}=2 B_{E}\left(\mathbf{U}^{1}, \mathbf{U}^{2}\right)+B_{(\mathbf{C}, \mathbf{N})}\left(\mathbf{U}^{2}, \mathbf{U}^{2}\right)
\end{aligned}
$$

Naturally, once $\mathcal{C}^{\infty}$ profiles have been built thanks to Theorem 4.15 and Proposition 4.16, regularity of these residual profiles is clear. The question we now need to face is to evaluate the size of these residuals as the intermediate time $T$ grows unboundedly.

Concerning the dominant profile $\mathbf{U}^{0}$, the components $\mathbf{u}^{0}$ and $\mathbf{N}^{0}$ are bounded uniformly in $T$, while the component $\mathbf{C}^{0}$ has exponential growth, i.e. $\mathbf{C}^{0}$ and all its derivatives have size $K_{1} \exp \left(K_{2} T\right)$ as $T$ increases, see Lemma 4.12 .

Concerning the first corrector $\mathbf{U}^{1}$, the components $\mathbf{u}_{0}^{1,0}, \Pi \mathbf{u}_{\text {osc }}^{1,0}$ and $\mathbf{N}_{0}^{1,0}+\mathbf{N}_{\text {space }}^{1,0}$ are precisely constructed so as to be sublinear in $T$, see Section 4.3. This is the key point. On top of that, the component $(1-\Pi) \mathbf{u}_{\text {osc }}^{1,0}$ is bounded, thanks to (40), the component $\mathbf{u}^{1,1}$ has exponential growth, thanks to (41), the component $\mathbf{N}^{1,1}$ has exponential growth, thanks to (48), the component $\mathbf{C}^{1,0}$ is bounded, thanks to (43), and the component $\mathbf{C}^{1,1}$ has exponential growth, thanks to (45) and (57) in conjunction with the Gronwall Lemma.

Concerning the second corrector $\mathbf{U}^{2}$, we know from (80) that $\mathbf{U}^{2}$ is an explicit, linear function of $\mathbf{U}^{0}$ and $\mathbf{U}^{1}$, functions that have at most exponential growth. Hence $\mathbf{U}^{2}$ has at most exponential growth in $T$. The only difficulty may then come from the component $\mathbf{U}^{2,0}$, whose exponential growth will not be eventually compensated by a decaying term $e^{-\sigma}$ or so. In that direction, we observe that $\mathbf{u}_{0}^{2,0}, \Pi \mathbf{u}_{\text {osc }}^{2,0}$, and $\mathbf{N}_{\alpha}^{2,0} \mathbf{1}_{\alpha \in \mathcal{C}_{0} \cup\{0\}}$ are conventionally chosen to vanish. On the other hand, the component $(1-\Pi) \mathbf{u}_{\text {osc }}^{2,0}$ is bounded thanks to (52) and to the boundedness of $\left(\mathbf{u}^{0}, \mathbf{N}^{0}, \mathbf{u}_{\text {osc }}^{1,0}\right)$, the component $\mathbf{C}^{2,0}$ is bounded thanks to (56), to the boundedness of $\mathbf{C}^{1,0}, \mathbf{u}^{0}, \mathbf{N}^{0}$, and to the sublinearity of $\mathbf{N}^{1,0}$, while the component $\mathbf{N}_{\alpha}^{2,0} \mathbf{1}_{\alpha \notin \mathcal{C}_{0} \cup\{0\}}$ is bounded thanks to (59), to the boundedness of $\mathbf{C}^{1,0}, \mathbf{u}^{0}, \mathbf{N}^{0}$, and to the sublinearity of $\mathbf{N}^{1,0}$.

As a conclusion, we have now established the

Lemma 5.1. Given the $\mathcal{C}^{\infty}$ profiles provided by Theorem 4.15 and Proposition 4.10 with the choice $s=+\infty$, the following result holds.

For all $\mu \in \mathbb{N}^{5+2 d}$, there are constants $K_{1}, K_{2}>0$ such that, uniformly on $\left[0, t_{\star}\right]_{t} \times \mathbb{R}_{T} \times \mathbb{R}_{x, y, z}^{3} \times \mathbb{T}_{\theta}^{2 d}$, for all $\kappa \in \mathbb{N}^{\star}, j=1,2,3$, we have

$$
\left|\partial_{t, T, x, y, z, \theta}^{\mu} r^{j, \kappa}(T)\right| \leq K_{1} e^{K_{2} T}, \text { and } \frac{1}{T}\left|\partial_{t, T, x, y, z, \theta}^{\mu} r^{j, 0}(T)\right|_{T \rightarrow+\infty}^{\longrightarrow} 0 .
$$

As a consequence, we get for the residual

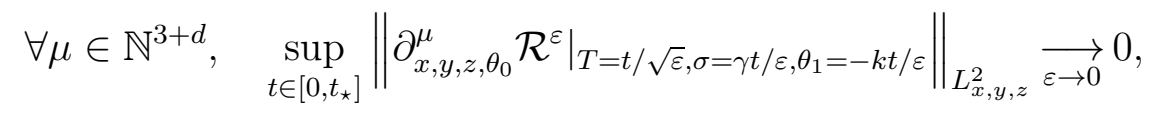

\subsection{Stability}

In this section, we prove our main Theorem. 
Theorem 5.2. Let the profiles $\mathbf{U}^{0}, \mathbf{U}^{1}, \mathbf{U}^{2} \in \mathcal{C}^{\infty}\left(\left[0, t_{\star}\right] \times\left[0,+\infty\left[{ }^{2}, H^{\infty}\left(\mathbb{R}^{3} \times \mathbb{T}^{2 d}\right)\right)\right.\right.$ be given by Theorem 4.15 and Proposition 4.10. They provide us with the approximate solution $\mathbf{U}_{\text {app }}^{\varepsilon}=\mathbf{U}_{\text {app }}^{\varepsilon}(t, x, y, z)$ given by equation (33).

Then, for any $s>(3+2 d) / 2$ and any familly $\left(\delta^{\varepsilon}\right)_{\varepsilon>0} \subset H^{s}\left(\mathbb{R}^{3} \times \mathbb{T}^{d}\right)$ such that $\left\|\delta^{\varepsilon}\right\|_{H^{s}} \underset{\varepsilon \rightarrow 0}{\longrightarrow} 0$, there is $\varepsilon_{0}>0$ such that for $\left.\left.\varepsilon \in\right] 0, \varepsilon_{0}\right]$, the Cauchy problem

$$
\left\{\begin{array}{l}
L\left(\partial_{t}, \partial_{x}, \frac{1}{\sqrt{\varepsilon}} \partial_{y}, \frac{1}{\sqrt{\varepsilon}} \partial_{z}\right) \mathbf{U}^{\varepsilon}=F^{\varepsilon}\left(\mathbf{U}^{\varepsilon}\right), \\
\mathbf{U}_{\mid t=0}^{\varepsilon}=\mathbf{U}_{\left.\mathrm{app}\right|_{t=0} ^{\varepsilon}}^{\varepsilon}+\delta^{\varepsilon}(x, y, z, k x / \varepsilon),
\end{array}\right.
$$

for the Maxwell-Bloch system (9) has a unique (mild) solution $\mathbf{U}^{\varepsilon}$ which belongs to $\mathcal{C}\left(\left[0, t_{\star}\right], H^{s}\left(\mathbb{R}^{3}\right)\right)$. Besides, for all $\mu \in \mathbb{N}^{3}$ such that $s-|\mu|>(3+2 d) / 2$, we have

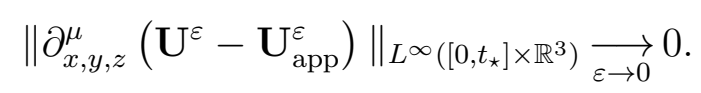

Proof. Standard results for symmetric hyperbolic systems ensure that, for $\varepsilon>0$ fixed, a unique mild solution $\mathbf{U}^{\varepsilon} \in \mathcal{C}\left(\left[0, t_{\varepsilon}\right], H^{s}\left(\mathbb{R}^{3}\right)\right)$ exists for some $t_{\varepsilon}>0$. The difficulty lies in bounding $t_{\varepsilon}$ from below.

We use a singular system method ( $c f$. [25]), and look for $\mathbf{U}^{\varepsilon}$ under the form of a profile, namely look for $\mathcal{U}^{\varepsilon}$ such that

$$
\mathbf{U}^{\varepsilon}(t, x, y, z)=\mathcal{U}^{\varepsilon}(t, x, y, z, k x / \varepsilon)
$$

where $\mathcal{U}^{\varepsilon}=\mathcal{U}^{\varepsilon}\left(t, x, y, z, \theta_{0}\right)$ corresponds to the following initial data, which is non-singular in $\varepsilon$ (this is a key point)

$$
\mathcal{U}^{\varepsilon}\left(0, x, y, z, \theta_{0}\right)=\left(\sum_{j=0}^{2} \sqrt{\varepsilon}^{j} \mathbf{U}^{j}\left(t, x, y, z, T, \sigma, \theta_{0}, \theta_{1}\right)+\delta^{\varepsilon}\left(t, x, y, z, \theta_{0}\right)\right)_{\left.\right|_{t=T=\sigma=0, \theta_{1}=0}} .
$$

It is then sufficient, for $\mathbf{U}^{\varepsilon}$ to be a solution to (9), that $\mathcal{U}^{\varepsilon}$ satisfies

$$
L\left(\partial_{t}, \partial_{x}+\frac{1}{\varepsilon} \partial_{\theta_{0}}, \frac{1}{\sqrt{\varepsilon}} \partial_{y}, \frac{1}{\sqrt{\varepsilon}} \partial_{z}\right) \mathcal{U}^{\varepsilon}=F^{\varepsilon}\left(\mathcal{U}^{\varepsilon}\right) .
$$

To go on with the analysis, we now set

$$
\mathcal{U}_{\text {app }}^{\varepsilon}\left(t, x, y, z, \theta_{0}\right)=\sum_{j=0}^{2} \sqrt{\varepsilon^{j}} U^{j}(t, x, y, z, T, \sigma, \theta)_{||_{T=t / \sqrt{\varepsilon}, \sigma=\gamma t / \varepsilon, \theta_{1}=k t / \varepsilon}} .
$$

Note in passing that the at-most-exponential growth in $T$ of the various profiles $\mathbf{U}^{j, \kappa}$ implies that the family $\left(\mathcal{U}_{\text {app }}^{\varepsilon}\right)_{\varepsilon}$ is bounded in $\mathcal{C}\left(\left[0, t_{\star}\right], H^{s}\left(\mathbb{R}^{3} \times \mathbb{T}^{d}\right)\right)$. We evaluate the difference

$$
\Delta^{\varepsilon}=\mathcal{U}^{\varepsilon}-\mathcal{U}_{\mathrm{app}}^{\varepsilon}
$$

In the next few lines, we may sometimes write $\Delta^{\varepsilon}=\left(\Delta_{\mathbf{u}}^{\varepsilon}, \Delta_{\mathbf{C}}^{\varepsilon}, \Delta_{\mathbf{N}}^{\varepsilon}\right)$, refering to the $\mathbf{u}, \mathbf{C}$ and $\mathbf{N}$ components of $\Delta^{\varepsilon}$, respectively. In any circumstance, we have $\Delta^{\varepsilon} \in \mathcal{C}\left(\left[0, t_{\varepsilon}\right], H^{s}\left(\mathbb{R}^{3} \times \mathbb{T}^{d}\right)\right)$, its initial value is $\left.\Delta^{\varepsilon}\right|_{t=0}=\delta^{\varepsilon}$, and $\Delta^{\varepsilon}$ satisfies

$$
L\left(\partial_{t}, \partial_{x}+\frac{1}{\varepsilon} \partial_{\theta_{0}}, \frac{1}{\sqrt{\varepsilon}} \partial_{y}, \frac{1}{\sqrt{\varepsilon}} \partial_{z}\right) \Delta^{\varepsilon}=F^{\varepsilon}\left(\mathcal{U}_{\mathrm{app}}^{\varepsilon}+\Delta^{\varepsilon}\right)-F^{\varepsilon}\left(\mathcal{U}_{\mathrm{app}}^{\varepsilon}\right)-\mathcal{R}_{\left.\right|_{T=t / \sqrt{\varepsilon}, \sigma=\gamma t / \varepsilon, \theta_{1}=k t / \varepsilon} ^{\varepsilon}} .
$$


Hence standard $H^{s}$ estimates provide

$$
\frac{1}{2} \frac{\mathrm{d}}{\mathrm{dt}}\left\|\Delta^{\varepsilon}\right\|_{H^{s}}^{2} \leq\left\langle F^{\varepsilon}\left(\mathcal{U}_{\mathrm{app}}^{\varepsilon}+\Delta^{\varepsilon}\right)-F^{\varepsilon}\left(\mathcal{U}_{\mathrm{app}}^{\varepsilon}\right) \mid \Delta^{\varepsilon}\right\rangle_{H^{s}}+\left\|\mathcal{R}^{\varepsilon}\right\|_{H^{s}}\left\|\Delta^{\varepsilon}\right\|_{H^{s}}
$$

Now, the evaluation of the scalar product $\left\langle F^{\varepsilon}\left(\mathcal{U}_{\text {app }}^{\varepsilon}+\Delta^{\varepsilon}\right)-F^{\varepsilon}\left(\mathcal{U}_{\text {app }}^{\varepsilon}\right) \mid \Delta^{\varepsilon}\right\rangle_{H^{s}}$ involves various terms that may be ordered in powers of $1 / \sqrt{\varepsilon}$ as seen by inspection of the bilinear function $F^{\varepsilon}$ in (9). The term carrying the weight $1 / \varepsilon$ is

$$
\frac{1}{\varepsilon}\left\langle-i\left[\Omega, \Delta_{\mathbf{C}}^{\varepsilon}\right]-\gamma \Delta_{\mathbf{C}}^{\varepsilon} \mid \Delta^{\varepsilon}\right\rangle_{H^{s}}=-\frac{\gamma}{\varepsilon}\left\langle\Delta_{\mathbf{C}}^{\varepsilon} \mid \Delta^{\varepsilon}\right\rangle_{H^{s}}=-\frac{\gamma}{\varepsilon}\left\|\Delta_{\mathbf{C}}^{\varepsilon}\right\|_{H^{s}}^{2},
$$

where the first equality comes from the fact that the operator $[\Omega,$.$] is skew-symmetric. To estimate$ the other terms, it is useful to keep in mind that $\mathcal{U}_{\text {app }}^{\varepsilon}$ is uniformly bounded in $H^{s}$ on the interval $\left[0, t_{*}\right]$, so there is a constant $C$, independent of $f$ and $\varepsilon$, such that for any $t$ and $\varepsilon$ we have $\left\|\mathcal{U}_{\text {app }}^{\varepsilon}(t)\right\|_{H^{s}} \leq C$. Using this piece of information, the term carrying the weight 1 in $\left\langle F^{\varepsilon}\left(\mathcal{U}_{\text {app }}^{\varepsilon}+\Delta^{\varepsilon}\right)-F^{\varepsilon}\left(\mathcal{U}_{\text {app }}^{\varepsilon}\right) \mid \Delta^{\varepsilon}\right\rangle_{H^{s}}$ is clearly bounded by

$$
C\left\|\Delta^{\varepsilon}\right\|_{H^{s}}^{2},
$$

for some constant $C$ independent of $t$ and $\varepsilon$, while the term carrying the weight $\sqrt{\varepsilon}$ in is clearly bounded by

$$
\sqrt{\varepsilon}\left\|\Delta^{\varepsilon}\right\|_{H^{s}}^{2}
$$

The more difficult term is the one carrying the weight $1 / \sqrt{\varepsilon}$. Carefully treating apart all occurences of the two terms $\Delta_{\mathbf{C}}^{\varepsilon}$ and $\mathbf{C}_{\mathrm{app}}^{\varepsilon}$, all terms being majorized in the most simple fashion, we recover that this contribution is upper-bounded by

$$
\frac{C}{\sqrt{\varepsilon}}\left(\left\|\Delta_{\mathbf{C}}^{\varepsilon}\right\|_{H^{s}}^{2}+\left\|\Delta_{\mathbf{C}}^{\varepsilon}\right\|_{H^{s}}\left\|\Delta^{\varepsilon}\right\|_{H^{s}}+\left\|\mathbf{C}_{\text {app }}^{\varepsilon}\right\|_{H^{s}}\left\|\Delta^{\varepsilon}\right\|_{H^{s}}^{2}+\left\|\mathbf{C}_{\text {app }}^{\varepsilon}\right\|_{H^{s}}\left\|\Delta_{\mathbf{C}}^{\varepsilon}\right\|_{H^{s}}\left\|\Delta^{\varepsilon}\right\|_{H^{s}}\right) .
$$

All in all we have eventually proved, gathering some terms for convenience,

$$
\begin{aligned}
\frac{\mathrm{d}}{\mathrm{dt}}\left\|\Delta^{\varepsilon}\right\|_{H^{s}}^{2} \leq & -\frac{\gamma}{\varepsilon}\left\|\Delta_{\mathbf{C}}^{\varepsilon}\right\|_{H^{s}}^{2}+\frac{C}{\sqrt{\varepsilon}}\left(\left\|\Delta_{\mathbf{C}}^{\varepsilon}\right\|_{H^{s}}^{2}+\left\|\Delta_{\mathbf{C}}^{\varepsilon}\right\|_{H^{s}}\left\|\Delta^{\varepsilon}\right\|_{H^{s}}\right) \\
& +\frac{C}{\sqrt{\varepsilon}}\left\|\mathbf{C}_{\mathrm{app}}^{\varepsilon}\right\|_{H^{s}}\left(\left\|\Delta_{\mathbf{C}}^{\varepsilon}\right\|_{H^{s}}\left\|\Delta^{\varepsilon}\right\|_{H^{s}}+\left\|\Delta^{\varepsilon}\right\|_{H^{s}}^{2}\right) \\
& +C\left\|\Delta^{\varepsilon}\right\|_{H^{s}}^{2}+\sqrt{\varepsilon}\left\|\Delta^{\varepsilon}\right\|_{H^{s}}^{2}+\left\|\mathcal{R}^{\varepsilon}\right\|_{H^{s}}\left\|\Delta^{\varepsilon}\right\|_{H^{s}} .
\end{aligned}
$$

Hence, there is an $\varepsilon_{0}$ such that for any $\varepsilon \leq \varepsilon_{0}$ we have,

$$
\frac{\mathrm{d}}{\mathrm{dt}}\left\|\Delta^{\varepsilon}\right\|_{H^{s}}^{2} \leq-\frac{1}{C \varepsilon}\left\|\Delta_{\mathbf{C}}^{\varepsilon}\right\|_{H^{s}}^{2}+\frac{C}{\sqrt{\varepsilon}}\left\|\Delta_{\mathbf{C}}^{\varepsilon}\right\|_{H^{s}}\left\|\Delta^{\varepsilon}\right\|_{H^{s}}+C \frac{\left\|\mathbf{C}_{\text {app }}^{\varepsilon}\right\|_{H^{s}}}{\sqrt{\varepsilon}}\left\|\Delta^{\varepsilon}\right\|_{H^{s}}^{2}+\left\|\mathcal{R}^{\varepsilon}\right\|_{H^{s}}\left\|\Delta^{\varepsilon}\right\|_{H^{s}} .
$$

Now, the two crucial ingredients are

$$
\sup _{t \in\left[0, t_{*}\right]}\left\|\mathcal{R}^{\varepsilon}\right\|_{H^{s}} \underset{\varepsilon \rightarrow 0}{\rightarrow} 0
$$

thanks to Lemma 5.1 , and

$$
\int_{0}^{t_{*}} \frac{\left\|\mathbf{C}_{\mathrm{app}}^{\varepsilon}(t)\right\|_{H^{s}}}{\sqrt{\varepsilon}} \mathrm{d} t \leq C \sqrt{\varepsilon}
$$


for some $C$ independent of $\varepsilon$. This crucial piece of information comes from the fact that

$$
\mathbf{C}_{\text {app }}^{\varepsilon}(t)=\left.\mathbf{C}^{0,1}\right|_{T=t / \sqrt{\varepsilon}} e^{-\gamma t / \varepsilon}+\left.\sqrt{\varepsilon} \mathbf{C}^{1,1}\right|_{T=t / \sqrt{\varepsilon}} e^{-\gamma t / \varepsilon}+\mathcal{O}(\varepsilon)
$$

which, in conjunction with the at-most-exponential growth of $\mathbf{C}^{0,1}$ and of $\mathbf{C}^{1,1}$ (see e.g. Lemma 4.12 and estimate (71)), provides

$$
\left\|\mathbf{C}_{\text {app }}^{\varepsilon}(t)\right\|_{H^{s}} \leq \exp \left(-\frac{t}{C \varepsilon}\right)+C \varepsilon,
$$

for some $C$ independent of $t \in\left[0, t_{*}\right]$ and $\varepsilon$.

At this stage, an easy argument using the Gronwall Lemma allows to deduce from (83), (84), and (85), that $\left\|\Delta^{\varepsilon}\right\|_{H^{s}}$ is bounded independently of $\varepsilon$ over the whole interval $\left[0, t_{*}\right]$. Repeting the Gronwall argument next shows that $\left\|\Delta^{\varepsilon}\right\|_{H^{s}}$ actually satisfies

$$
\left\|\Delta^{\varepsilon}\right\|_{H^{s}} \leq\left\|\delta^{\varepsilon}\right\|_{H^{s}} \exp \left(C \int_{0}^{t}\left(\frac{\left\|\mathbf{C}_{\mathrm{app}}^{\varepsilon}\right\|_{H^{s}}}{\sqrt{\varepsilon}}+\left\|\mathcal{R}^{\varepsilon}\right\|_{H^{s}}\right) d t^{\prime}\right) \underset{\varepsilon \rightarrow 0}{\longrightarrow} 0, \quad \text { uniformly on }\left[0, t_{*}\right] .
$$

We skip the whole Gronwall-like argument. Estimate (86) now induces, by Sobolev's injection, $L^{\infty}$ convergence to zero for profiles $\Delta^{\varepsilon}=\mathcal{U}^{\varepsilon}-\mathcal{U}_{\text {app }}^{\varepsilon}$, and thus $L^{\infty}$ convergence to zero for the original functions $\mathbf{U}-\mathbf{U}_{\mathrm{app}}^{\varepsilon}$, since $\left\|\mathbf{U}-\mathbf{U}_{\mathrm{app}}^{\varepsilon}\right\|_{L_{t, x, y, z}^{\infty}}=\left\|\mathcal{U}^{\varepsilon}-\mathcal{U}_{\mathrm{app}}^{\varepsilon}\right\|_{L_{t, x, y, z, \theta_{0}}^{\infty}}$.

\section{The Transverse Magnetic case}

\subsection{The system}

In this section, we present the previous WKB in the particular Transverse Magnetic case, when fields take the form

$$
B=\left(\begin{array}{c}
B_{x} \\
B_{y} \\
0
\end{array}\right)=B(t, x, y), \quad E=\left(\begin{array}{c}
0 \\
0 \\
E
\end{array}\right)=E(t, x, y)
$$

with the additional common assumption ( $c f$. 34] that the polarization operator $\Gamma$ has entries parallel to $E$, namely

$$
\forall m, n \in\{1, \ldots, N\}, \quad \Gamma(m, n)=\left(\begin{array}{c}
0 \\
0 \\
\Gamma(m, n)
\end{array}\right) .
$$

Maxwell-Bloch system then reads

$$
\begin{aligned}
& \partial_{t} B_{x}^{\varepsilon}+\frac{1}{\sqrt{\varepsilon}} \partial_{y} E^{\varepsilon}=0, \\
& \partial_{t} B_{y}^{\varepsilon}+\frac{1}{\sqrt{\varepsilon}} \partial_{x} E^{\varepsilon}=0, \\
& \partial_{t} E^{\varepsilon}-\partial_{x} B_{y}^{\varepsilon}+\partial_{y} B_{x}^{\varepsilon}=\frac{i}{\sqrt{\varepsilon}} \operatorname{Tr}\left(\Gamma \Omega_{\gamma} \mathbf{C}^{\varepsilon}\right)-i E^{\varepsilon} \operatorname{Tr}\left(\Gamma\left[\Gamma, \mathbf{C}^{\varepsilon}+\mathbf{N}^{\varepsilon}\right]\right)-\sqrt{\varepsilon} \operatorname{Tr}\left(\Gamma W \sharp \mathbf{N}^{\varepsilon}\right), \\
& \partial_{t} \mathbf{C}^{\varepsilon}=-\frac{i}{\varepsilon} \Omega_{\gamma} \mathbf{C}^{\varepsilon}+\frac{i}{\sqrt{\varepsilon}} E^{\varepsilon}\left[\Gamma, \mathbf{C}^{\varepsilon}+\mathbf{N}^{\varepsilon}\right]_{\mathrm{od}}, \\
& \partial_{t} \mathbf{N}^{\varepsilon}=\frac{i}{\sqrt{\varepsilon}} E^{\varepsilon}\left[\Gamma, \mathbf{C}^{\varepsilon}\right]_{\mathrm{d}}+W \sharp \mathbf{N}^{\varepsilon} .
\end{aligned}
$$




\subsection{The Ansatz}

As stressed in Remark 3.2, the introduction of an intermediate time $T=t / \sqrt{\varepsilon}$ is not necessary here (see also Remark 6.1 below). In order to simplify computations, we also restrict here our attention to the case of prepared data, which corresponds to the case when

$$
\left.\mathbf{C}^{\varepsilon}\right|_{t=0}=0
$$

This second simplification allows us not to use the variable $\sigma=\gamma t / \varepsilon$ in the sequel. In a nutshell, we here consider the simplified Ansatz

$$
\mathbf{U}_{\text {app }}^{\varepsilon}(t, x, y)=\sum_{j=0}^{2} \sqrt{\varepsilon}^{j} \mathbf{U}^{j}(t, x, y, \theta)_{\mid \theta=(k x / \varepsilon,-k t / \varepsilon}, \quad \mathbf{U}^{j}(t, x, y, \theta)=\sum_{\alpha \in \mathbb{Z}^{2 d}} \sum_{\kappa \in \mathbb{N}} \mathbf{U}_{\alpha}^{j}(t, x, y) e^{i \alpha \cdot \theta} .
$$

The characteristic sets $\mathcal{C}_{0}, \mathcal{C}_{+}, \mathcal{C}_{-}$and the resonant set $\mathcal{R}(k)$ are the same as before.

\subsection{WKB expansions}

In this setting, vanishing of the terms $r^{-2}, r^{-1}$ and $r^{0}$ from Proposition 4.2 reduces to

$$
\begin{aligned}
& \left\{\begin{array}{l}
-k \cdot \partial_{\theta_{1}} B_{x}^{0}=0 \\
-k \cdot \partial_{\theta_{1}} B_{y}^{0}-k \cdot \partial_{\theta_{0}} E^{0}=0 \\
-k \cdot \partial_{\theta_{1}} E^{0}-k \cdot \partial_{\theta_{0}} B_{y}^{0}=0 \\
\left(i \Omega_{\gamma}-k \cdot \partial_{\theta_{1}}\right) \mathbf{C}^{0}=0 \\
-k \cdot \partial_{\theta_{1}} \mathbf{N}^{0}=0
\end{array}\right. \\
& \left\{\begin{array}{l}
-k \cdot \partial_{\theta_{1}} B_{x}^{1}+\partial_{y} E^{0}=0 \\
-k \cdot \partial_{\theta_{1}} B_{y}^{1}-k \cdot \partial_{\theta_{0}} E^{1}=0 \\
-k \cdot \partial_{\theta_{1}} E^{1}-k \cdot \partial_{\theta_{0}} B_{y}^{1}+\partial_{y} B_{x}^{0}=0 \\
\left(i \Omega_{\gamma}-k \cdot \partial_{\theta_{1}}\right) \mathbf{C}^{1}=i E^{0}\left[\Gamma, \mathbf{C}^{0}+\mathbf{N}^{0}\right]_{\mathrm{od}} \\
-k \cdot \partial_{\theta_{1}} \mathbf{N}^{1}=i E^{0}\left[\Gamma, \mathbf{C}^{0}\right]_{\mathrm{d}}
\end{array}\right. \\
& \left\{\begin{array}{l}
-k \cdot \partial_{\theta_{1}} B_{x}^{2}+\partial_{y} E^{1}+\partial_{t} B_{x}^{0}=0, \\
-k \cdot \partial_{\theta_{1}} B_{y}^{2}-k \cdot \partial_{\theta_{0}} E^{2}+\partial_{t} B_{y}^{0}-\partial_{x} E^{0}=0, \\
-k \cdot \partial_{\theta_{1}} E^{2}-k \cdot \partial_{\theta_{0}} B_{y}^{2}+\partial_{y} B_{x}^{1}+\partial_{t} E^{0}-\partial_{x} B_{y}^{0}=i \operatorname{Tr}\left(\Gamma \Omega_{\gamma} \mathbf{C}^{1}\right)-i E^{0} \operatorname{Tr}\left(\Gamma\left[\Gamma, \mathbf{C}^{0}+\mathbf{N}^{0}\right]\right), \\
\left(i \Omega_{\gamma}-k \cdot \partial_{\theta_{1}}\right) \mathbf{C}^{2}+\partial_{t} \mathbf{C}^{0, \kappa}=i E^{0}\left[\Gamma, \mathbf{C}^{1}+\mathbf{N}^{1}\right]_{\mathrm{od}}+i E^{1}\left[\Gamma, \mathbf{C}^{0}+\mathbf{N}^{0}\right]_{\mathrm{od}}, \\
-k \cdot \partial_{\theta_{1}} \mathbf{N}^{2}+\partial_{t} \mathbf{N}^{0}=i E^{0}\left[\Gamma, \mathbf{C}^{1}\right]_{\mathrm{d}}+i E^{1}\left[\Gamma, \mathbf{C}^{0}\right]_{\mathrm{d}}+W \sharp \mathbf{N}^{0} .
\end{array}\right.
\end{aligned}
$$

According to (89), the polarization conditions from equations (35)-(37) become, with the notations from Definition 4.7

$$
\begin{aligned}
& B_{x, \text { time }}^{0}=0, \\
& \forall \alpha \notin \mathcal{C}_{+} \cup \mathcal{C}_{-} \cup\{0\}, \quad B_{y, \alpha}^{0}=E_{\alpha}^{0}=0, \\
& \forall \alpha \in \mathcal{C}_{+} \cup \mathcal{C}_{-}, \quad B_{y, \alpha}^{0}=\mp E_{\alpha}^{0}, \\
& \mathbf{C}^{0}=0 \\
& \mathbf{N}_{\text {time }}^{0}=0
\end{aligned}
$$


Here, the projector $\Pi$ from Definition 4.3 is given explicitly, and the "prepared data" condition is coherent with the vanishing of $\mathbf{C}^{0}$.

From (90), we get for the average $\mathbf{u}_{0}^{0}$ a version of the evolution equation at intermediate scale (38) under the form

$$
\partial_{y} E_{0}^{0}=\partial_{y} B_{x, 0}^{0}=0,
$$

which leads to polarization consitions, instead of evolution equations, namely

$$
E_{0}^{0}=B_{x, 0}^{0}=0 .
$$

The oscillating part of (90) produces the following transcription of the polarization condition (40) for the first corrector $\mathbf{u}^{1}$

$$
\left\{\begin{array}{l}
\forall \alpha \notin \mathcal{C} \cup\{0\}, \quad \mathbf{u}_{\alpha}^{1}=0, \\
\forall \alpha \in \mathcal{C}_{0}, \quad E_{\alpha}^{1}=0, \quad B_{y, \alpha}^{1}=\frac{1}{i k \cdot \alpha_{0}} \partial_{y} B_{x, \alpha}^{0}, \\
\forall \alpha \in \mathcal{C}_{ \pm}, \quad B_{x, \alpha}^{1}=\frac{1}{i k \cdot \alpha_{1}} \partial_{y} E_{\alpha}^{0}, \quad B_{y, \alpha}^{1}=\mp E_{\alpha}^{1} .
\end{array}\right.
$$

For coherences and populations, equation (43) is unchanged,

$$
\mathbf{C}^{1}=i\left(i \Omega_{\gamma}-k \cdot \partial_{\theta_{1}}\right)^{-1} E^{0}\left[\Gamma, \mathbf{N}^{0}\right]_{\mathrm{d}},
$$

and equation (48) becomes

$$
\mathbf{N}_{\text {time }}^{1}=0 .
$$

Next, the average of fields equations in (91) is equivalent to

$$
\partial_{y} E_{0}^{1}=0, \quad \text { i.e. } \quad E_{0}^{1}=0
$$

(a polarization condition, again, instead of evolution as in equation (50)),

$$
\partial_{t} B_{y, 0}^{0}=0
$$

(playing the role of equation (65)), and

$$
\partial_{y} B_{x, 0}^{1}-\partial_{x} B_{y, 0}^{0}=-i\left(E^{0} \operatorname{Tr}\left(\Gamma\left[\Gamma, \mathbf{N}^{0}\right]\right)\right)_{0},
$$

which reduces to

$$
\partial_{y} B_{x, 0}^{1}-\partial_{x} B_{y, 0}^{0}=0,
$$

thanks to polarizations conditions and spectral properties (93), (96) and (97).

Remark 6.1. Since $B_{y, 0}^{0}$ does not depend on timet (according to equation (102)), we may impose (as in equation (103) ) that $\partial_{x} B_{y, 0}^{0}$ be the $y$-derivative of an $H^{s}$ function for all times, simply by requiring this condition be satisfied at $t=0$. But in the general three-dimensional framework, $\partial_{t} B_{y, 0}^{0}=$ 0 is not given a priori, and we need the addition of the intermediate variable $T$ to perform the analysis of Section 4.3. Omitting this intermediate time leads, in the three-dimensional case, to the overdetermined (and ill-posed) system (38), (50)

$$
M_{2}\left(0, \partial_{y}, \partial_{z}\right) \mathbf{u}_{0}^{0}=0, \quad M_{2}\left(0, \partial_{y}, \partial_{z}\right) \mathbf{u}_{0}^{1}=-M_{1}\left(\partial_{t}, \partial_{x}\right) \mathbf{u}_{0}^{0}
$$


Oscillations in (91) are analyzed as follows. The polarization (52) for $\mathbf{u}_{\mathrm{osc}}^{2}$ splits into

$$
B_{x, \text { time }}^{2}=\left(k \cdot \partial_{\theta_{1}}\right)^{-1} \partial_{y} E_{\text {time }}^{1},
$$

and

$$
\left\{\begin{array}{c}
\forall \alpha \in \mathcal{C}_{ \pm}, \quad 2 i\left(B_{y, \alpha}^{2} \pm E_{\alpha}^{2}\right)= \\
\forall \alpha \notin \mathcal{C}_{+} \cup \mathcal{C}_{-} \cup\{0\}, \quad\left(\begin{array}{c}
B_{y, \alpha}^{2} \\
E_{\alpha}^{2}
\end{array}\right)=\frac{1}{\left(k \cdot \partial_{\theta_{1}}\right)_{y}^{2}-\left(k \cdot \alpha_{1}\right)^{2}} \times \\
\quad \times\left(\begin{array}{cc}
k \cdot \alpha_{1} & -k \cdot \alpha_{0} \\
-k \cdot \alpha_{0} & k \cdot \alpha_{1}
\end{array}\right)\left(\begin{array}{c}
0 \\
\partial_{y} B_{x, \alpha}^{1}+i \operatorname{Tr}\left(\Gamma \Omega_{\gamma}\left(E^{0} \mathbf{N}^{0}-\mathbf{C}^{1}\right)_{\alpha}\right)
\end{array}\right),
\end{array}\right.
$$

where $B_{x, \alpha}^{1}$ vanishes for $\alpha \notin \mathcal{C}$ (because of (98)), and may be chosen arbitrarily when $\alpha \in \mathcal{C}_{0}$. The coherence $\mathbf{C}^{1}$ is given by equation (99). Then, the evolution equation (66) with respect to the slow time $t$ corresponds to

$$
\partial_{t} B_{x, \text { space }}^{0}=0, \quad \text { together with } \quad \partial_{t} B_{x, 0}^{0}=0,
$$

and, using (99), to

$$
\forall \alpha \in \mathcal{C}_{ \pm}, \quad 2\left(\partial_{t} \pm \partial_{x}\right) E_{\alpha}^{0}+\frac{1}{i k \cdot \alpha_{1}} \partial_{y}^{2} E_{\alpha}^{0}=i \operatorname{Tr}\left(\Gamma \Omega_{\gamma} \mathbf{C}_{\alpha}^{1}\right)-i\left(E^{0} \operatorname{Tr}\left(\Gamma\left[\Gamma, \mathbf{N}^{0}\right]\right)\right)_{\alpha}
$$

or (with $\mathbf{C}_{\alpha}^{1}$ from (99)), in other words

$$
2\left(\partial_{t}+\mathrm{v}\left(\mathrm{D}_{\theta}\right) \partial_{x}\right) E^{0}+\partial_{\theta_{1}}^{-1} \partial_{y}^{2} E^{0}=i \operatorname{Tr}\left(\Gamma\left(i \Omega_{\gamma}\left(i \Omega_{\gamma}-k \cdot \partial_{\theta_{1}}\right)^{-1}-1\right)\left(E^{0}\left[\Gamma, \mathbf{N}^{0}\right]\right)\right),
$$

(in place of equation (51)), with $\mathrm{v}\left(\mathrm{D}_{\theta}\right)$ given in Lemma 4.5.

For coherences, we get

$$
\mathbf{C}^{2}=i\left(i \Omega_{\gamma}-k \cdot \partial_{\theta_{1}}\right)^{-1}\left(E^{1}\left[\Gamma, \mathbf{N}^{0}\right]_{\text {od }}+E^{0}\left[\Gamma, \mathbf{C}^{1}+\mathbf{N}^{1}\right]_{\text {od }}\right),
$$

and for populations, we have

$$
\mathbf{N}_{\text {time }}^{2}=-i\left(k \cdot \partial_{\theta_{1}}\right)^{-1}\left(E^{0}\left[\Gamma, \mathbf{C}^{1}\right]_{\mathrm{od}}\right)_{\text {time }},
$$

and

$$
\partial_{t} \mathbf{N}^{0}=W_{\sharp} \mathbf{N}^{0}+i\left(E^{0}\left[\Gamma, \mathbf{C}^{1}\right]_{\mathrm{d}}\right)_{\text {space }}+i\left(E^{0}\left[\Gamma, \mathbf{C}^{1}\right]_{\mathrm{d}}\right)_{0},
$$

i.e.

$$
\begin{aligned}
\partial_{t} \mathbf{N}^{0}=W_{\sharp} \mathbf{N}^{0} & -\left(E^{0}\left[\Gamma,\left(i \Omega_{\gamma}-k \cdot \partial_{\theta_{1}}\right)^{-1}\left(E^{0}\left[\Gamma, \mathbf{N}^{0}\right]_{\mathrm{od}}\right)\right]_{\mathrm{d}}\right)_{\text {space }} \\
& -\left(E^{0}\left[\Gamma,\left(i \Omega_{\gamma}-k \cdot \partial_{\theta_{1}}\right)^{-1}\left(E^{0}\left[\Gamma, \mathbf{N}^{0}\right]_{\mathrm{od}}\right)\right]_{\mathrm{d}}\right)_{0} .
\end{aligned}
$$

\subsection{Conclusion in the TM case}

The above computations provide us with a set of profile equations leading to a local in time, smooth approximate solution $\mathbf{U}_{\text {app }}^{\varepsilon}$ as in (88): 
- The leading profile $\left(\mathbf{u}^{0}, \mathbf{C}^{0}, \mathbf{N}^{0}\right)$ is given by polarizations (92), (93), (94), (95) $\left(\mathbf{C}^{0}=0\right)$, (96), as well as (97) (for average of fields, instead of an evolution at the intermediate scale). They also satisfy an evolution with respect to time $t$, given by the trivial equations (102) and (106), namely

$$
\partial_{t} B_{y, 0}^{0}=\partial_{t} B_{x, \mathrm{sp}}^{0}=0
$$

by a nonlinear Schrödinger equation (107), and by the Boltzmann equation (110). As quoted in Remark 6.1, this a priori overdetermined set of equations is in fact well-posed because of its scalar structure. We stress the fact that no rectification occurs for fields at leading order: the only nonvanishing average $B_{y, 0}^{0}$ is constant in time.

- Correctors are partially determined by polarizations, namely (98), (101), (103) for $\mathbf{u}^{1}$, (99) for $\mathbf{C}^{1}$, (100) for $\mathbf{N}^{1}$, (104), (105) for $\mathbf{u}^{2}$, (108) for $\mathbf{C}^{2}$, and (109) for $\mathbf{N}^{2}$. Parts of the correctors that are not submitted to these constraints may be chosen equal to zero.

The Ansatz

$$
\mathbf{U}_{\text {app }}^{\varepsilon}(t, x, y)=\mathcal{U}_{\text {app }}^{\varepsilon}(t, x, y, \theta)_{\left.\right|_{\theta=(k / \varepsilon,-k t / \varepsilon)}}
$$

is consistant with system (87). We easily prove the

Lemma 6.2. Given the $\mathcal{C}^{\infty}$ profiles above, define the residual $\mathcal{R}^{\varepsilon}$ as in Proposition 4.2 . Then, for all $\mu \in \mathbb{N}^{2+d}$, there is $K>0$ such that:

$$
\sup _{t \in\left[0, t_{\star}\right]}\left\|\left.\partial_{x, y, \theta_{0}}^{\mu} \mathcal{R}^{\varepsilon}\right|_{\theta_{1}=-k t / \varepsilon}\right\|_{L_{x, y}^{2}} \leq K \sqrt{\varepsilon}
$$

Note that for prepared data, no initial layer is created at leading order, so that we get a $\mathcal{O}(\sqrt{\varepsilon})$ estimate instead of the $o(1)$ in Lemma 5.1.

By the same technique as in Section 5.2, we get finally

Theorem 6.3. Given the smooth profiles above on $\left[0, t_{\star}\right]$, for $s>(2+d) / 2$ and any familly $\left(\delta^{\varepsilon}\right)_{\varepsilon>0} \subset$ $H^{s}\left(\mathbb{R}^{2} \times \mathbb{T}^{d}\right)$ such that $\left\|\delta^{\varepsilon}\right\|_{H^{s}}=\mathcal{O}(\sqrt{\varepsilon})$, there is $\varepsilon_{0}>0$ such that for $\left.\left.\varepsilon \in\right] 0, \varepsilon_{0}\right]$, the Cauchy problem for Transverse Magnetic Maxwell-Bloch system (87), with initial data $\left.\mathbf{U}_{\text {app }}^{\varepsilon}\right|_{t=0}+\delta^{\varepsilon}(x, y, k x / \varepsilon)$, has a unique solution $\mathbf{U}^{\varepsilon} \in \mathcal{C}\left(\left[0, t_{\star}\right], H^{s}\left(\mathbb{R}^{2}\right)\right)$, and for all $\mu \in \mathbb{N}^{2}$ such that $s-|\mu|>(2+d) / 2$, there is $K>0$ such that

$$
\left\|\partial_{x, y}^{\mu}\left(\mathbf{U}^{\varepsilon}-\mathbf{U}_{\mathrm{app}}^{\varepsilon}\right)\right\|_{L^{\infty}\left(\left[0, t_{\star}\right] \times \mathbb{R}^{2}\right)} \leq K \sqrt{\varepsilon}
$$

\section{References}

[1] S. Alinhac and P. Gérard. Opérateurs pseudo-différentiels et théorème de Nash-Moser. InterEditions, 1991.

[2] D. Benedetto, F. Castella and R. Esposito and M. Pulvirenti. Some considerations on the derivation of the nonlinear quantum Boltzmann equation. J. Stat. Phys. 116 (2004), 381-410.

[3] B. Bidégaray-Fesquet, A. Bourgeade and D. Reignier. Introducing physical relaxation terms in Bloch equations. J. Comput. Phys. 170 (2001), 603-613.

[4] B. Bidégaray-Fesquet. De Maxwell-Bloch à Schrödinger non linéaire: une hiérarchie de modèles en optique quantique. Collection Mathématiques et Applications, vol. 49, Springer,2006.

[5] F. Castella, P. Degond and T. Goudon. Asymptotic problems for laser-matter modeling: quantum and classical models. Nonlinearity 20 (2007), 1677-1720. 
[6] B. Bidégaray-Fesquet, F. Castella and P. Degond. From Bloch model to the rate equations. Discr. Cont. Dyn. Syst. 11 (2004), 1-26.

[7] B. Bidégaray-Fesquet, F. Castella, E. Dumas and M. Gisclon. From Bloch model to the rate equations II: the case of almost degenerate energy levels. Math. Models Methods Appl. Sci. 14 (2004), no. 12, 1785-1817.

[8] A. Bohm. Quantum Mechanics. Texts and monographs in Physics, Springer-Verlag, 1979.

[9] T. Boucheres, T. Colin, B. Nkonga, B. Texier and A. Bourgeade. Study of a mathematical model for stimulated Raman scattering. Math. Models Methods Appl. Sci. 14 (2004), no. 2, 217-252.

[10] R.W. Boyd. Nonlinear Optics. Academic Press, 1992.

[11] F. Castella. On the derivation of a Quantum Boltzmann Equation from the periodic von Neumann equation. Mod. Math. An. Num. 33 (1999), no. 2, 329-349.

[12] F. Castella. From the von Neumann equation to the Quantum Boltzmann equation in a deterministic framework. J. Stat. Phys. 104 (2001), no. 1/2, 387-447.

[13] F. Castella. From the von Neumann equation to the Quantum Boltzmann equation II: identifying the Born series. J. Stat. Phys. 106 (2002), no. 5/6, 1197-1220.

[14] F. Castella and A. Plagne. A distribution result for slices of sums of squares. Math. Proc. Cambridge Philos. Soc. 132 (2002), no. 1, 1-22.

[15] F. Castella and A. Plagne. Non-derivation of the Quantum Boltzmann equation from the periodic Schrödinger equation. Indiana Univ. Math. J. 51 (2003), 963-1016.

[16] C. Cohen-Tannoudji, J. Dupont-Roc and G. Grynberg. Processus d'interaction entre photons et atomes. Savoirs actuels, Intereditions/Editions du CNRS, 1988.

[17] P. Donnat. Quelques contributions mathématiques en optique non linéaire. PhD Thesis, 1994.

[18] P. Donnat, J.L. Joly, G. Métivier and J. Rauch. Diffractive nonlinear geometric optics. Séminaire Equations aux Dérivées Partielles, Ecole Polytechnique, Palaiseau, 1995-1996.

[19] E. Dumas. Periodic multiphase nonlinear diffractive optics with curved phases. Indiana Univ. Math. J. 52 (2003), no. 3, 769-810.

[20] E. Dumas. Nonlinear diffractive optics with curved phases: beam dispersion and transition between light and shadow. Asymptot. Anal. 38 (2004), no. 1, 47-91.

[21] E. Dumas. About nonlinear geometric optics. Boletin de SEMA 35 (2006), 7-42.

[22] L. Erdös and H.T. Yau. Linear Boltzmann equation as the weak coupling limit of a random Schrödinger equation. Comm. Pure Appl. Math. 53 (2000), no. 6, 667-735.

[23] J.K. Hunter. Transverse diffraction of nonlinear waves and singular rays. SIAM J. Appl. Math. 48 (1988), no. $1,1-37$.

[24] J.L. Joly, G. Métivier and J. Rauch. Generic rigorous asymptotic expansions for weakly nonlinear multidimensional oscillatory waves. Duke Math. J. 70 (1993), no. 2, 373-404. 
[25] J.L. Joly, G. Métivier and J. Rauch. Coherent and focusing multidimensional nonlinear geometric optics. Ann. scient. Ec. Norm. Sup. 28 (1995), 51-113.

[26] J.L. Joly, G. Métivier and J. Rauch. Diffractive nonlinear geometric optics with rectification. Indiana Univ. Math. J. 47 (1998), no. 4, 1167-1241.

[27] J.L. Joly, G. Métivier and J. Rauch. Recent results in non-linear geometric optics. in: Hyperbolic problems: theory, numerics, applications, Vol. II, 723-736. Internat. Ser. Numer. Math. 130, Birkhäuser, Basel, 1999.

[28] J.L. Joly, G. Métivier and J. Rauch. Transparent nonlinear geometric optics and Maxwell-Bloch equations. J. Differential Equations 166 (2000), no. 1, 175-250.

[29] J.B. Keller and G. Papanicolaou and L. Ryzhik. Transport equations for elastic and other waves in random media. Wave Motion 24 (1996), no. 4, 327-370.

[30] D. Lannes. Dispersive effects for nonlinear geometrical optics with rectification. Asympt. Anal. 18 (1998), no. 1-2, 111-146.

[31] D. Lannes. Secular growth estimates for hyperbolic systems. J. Differential Equations. 190 (2003), no. 2, 466-503.

[32] P.D. Lax. Asymptotic solutions of oscillatory initial value problems. Duke Math. J. 24 (1957), 627-646.

[33] R. Loudon. The quantum theory of light. Clarendon Press, Oxford, 1991.

[34] A.C. Newell and J.V. Moloney. Nonlinear optics. Addison-Wesley Publishing Company Advanced Book Program, Redwood City, CA, 1992.

[35] F. Nier. A semi-classical picture of quantum scattering. Ann. Sci. Ec. Norm. Sup., 4. Sér., 29 (1996), no. 2, 149-183.

[36] R. Pantell and H. Puthoff. Fundamentals of quantum electronics. Wiley and Sons Inc., N.Y., 1969.

[37] M. Sargent, M.O. Scully and W.E. Lamb. Laser Physics. Addison-Wesley, 1977.

[38] H. Spohn. Derivation of the transport equation for electrons moving through random impurities. J. Stat. Phys. 17 (1977), no. 6, 385-412.

[39] H. Spohn. Kinetic equations from Hamiltonian dynamics: Markovian limits. Rev. Mod. Phys. 52 (1980), no. 3, 569-615.

[40] H. Spohn. Large Scale Dynamics of interacting particles. Springer, Berlin, 1991.

[41] B. Texier. The short-wave limit for nonlinear, symmetric, hyperbolic systems. Adv. Differential Equations 9 (2004), 1-52. 